\title{
Extrapolating Long-Maturity Bond Yields for Financial Risk Measurement
}

\author{
Jens H. E. Christensen \\ Jose A. Lopez \\ Paul L. Mussche \\ Federal Reserve Bank of San Francisco \\ March 2019 \\ Working Paper 2018-09 \\ https://www.frbsf.org/economic-research/publications/working-papers/2018/09/
}

\section{Suggested citation:}

Christensen, Jens H. E., Jose A. Lopez, Paul L. Mussche. 2019. "Extrapolating LongMaturity Bond Yields for Financial Risk Measurement,” Federal Reserve Bank of San Francisco Working Paper 2018-09. https://doi.org/10.24148/wp2018-09

The views in this paper are solely the responsibility of the authors and should not be interpreted as reflecting the views of the Federal Reserve Bank of San Francisco or the Board of Governors of the Federal Reserve System. 


\title{
Extrapolating Long-Maturity Bond Yields for Financial Risk Measurement
}

\author{
Jens H. E. Christensen \\ Jose A. Lopez \\ Paul L. Mussche \\ Federal Reserve Bank of San Francisco \\ 101 Market Street \\ San Francisco, CA 94105
}

\begin{abstract}
Insurance companies and pension funds have liabilities far into the future and typically well beyond the longest maturity bonds trading in fixed-income markets. Such longlived liabilities still need to be discounted, and yield curve extrapolations based on the information in observed yields can be used. We use dynamic Nelson-Siegel (DNS) yield curve models for extrapolating risk-free yield curves for Switzerland, Canada, France, and the U.S. We find slight biases in extrapolated long bond yields of a few basis points. In addition, the DNS model allows the generation of useful financial risk metrics, such as ranges of possible yield outcomes over projection horizons commonly used for stress-testing purposes. Therefore, we recommend using DNS models as a simple tool for generating extrapolated yields for long-term interest rate risk management.
\end{abstract}

JEL Classification: E43, E47, G12, G22, G28

Keywords: term structure modeling, capital regulation of insurance companies

We thank participants at the 2017 Federal Reserve System Committee Meeting on Financial Institutions, Regulation, and Markets Program, including our discussant Peter Van Tassel, for helpful comments. Furthermore, we thank Eric Fischer and Nikola Mirkov for helpful comments and suggestions on an earlier draft of the paper. Finally, we thank Nikola Mirkov and Thomas Nitschka for sharing the Swiss yield data with us. The views in this paper are solely the responsibility of the authors and not necessarily those of the Federal Reserve Bank of San Francisco or the Federal Reserve System.

This version: March 22, 2019. 


\section{Introduction}

Insurance companies and other financial institutions can have liabilities very far into the future; for example, some of their products offer customers payments for the rest of their lives starting at a certain date in the future, contingent upon survival. Because these claims are not expected for many years and generally cannot be accelerated, their effective maturity can be very long relative to the assets the companies hold. To calculate various portfolio performance and risk measures, such future cash flows need to be discounted using either a risk-free yield curve or an appropriately risky yield curve. However, yields based on liquid securities (such as government bonds) that might be used for this discounting typically do not exist for such long maturities.

For example, in the U.S., the longest maturity for Treasury bonds is typically thirty years, although some longer ones were issued in the past (Garbade 2017a,b). While dollarbased interest rate swaps and related derivative contracts are written with maturities longer than thirty years, they are not widely traded and are likely affected by transaction-specific features. In contrast, certain countries - such as Switzerland, Canada, and France-have issued government securities with maturities up to fifty years. In this paper, we address the question of how to create extrapolations of risk-free yield curves beyond the maximum maturity available - the so-called "last liquid point" (LLP) - as required for managing such long-term interest rate risks.

Other extrapolation techniques are currently in use. The predominant regulatory approach for projecting risk-free yields beyond the LLP is set by the European Insurance and Occupational Pensions Authority (EIOPA). As described in their April 2017 press release, the so-called "ultimate forward rate" (UFR) for each currency's risk-free rate is set annually to be the sum of an expected real rate, which is set to the same for all countries, and an expected currency-specific inflation rate. The expected real rate is set as the simple average of the annual real interest rates of Belgium, Germany, France, Italy, the Netherlands, the United Kingdom, and the United States since 1961. A currency's expected inflation rate is set equal to its central bank's inflation target, and in the absence of such a target, it is set to $2 \%$ by default. The UFRs are updated annually, but changed only if the latest UFR value differs from the current applied value by more than \pm 15 basis points, at which point the UFR is updated by only 15 basis points. ${ }^{1}$ This approach has several advantages: it is clearly grounded in the observable historical data; it encompasses a set of developed economies that represent a large proportion of global economic activity and global government bond issuance; and it allows for dynamic adjustments over time, particularly in light of changes in the so-called "natural" rate of interest. ${ }^{2}$

\footnotetext{
${ }^{1}$ Some national insurance regulatory bodies have implemented alternative UFR methodologies within their jurisdictions. See Zigraiova and Jakubik (2017) for a further discussion of the EIOPA algorithm and certain national alternatives.

${ }^{2}$ See Laubach and Williams (2016) as well as Christensen and Rudebusch (2019) for recent research into
} 
An important shortcoming of this approach, however, is that it is purely backward-looking and does not incorporate market expectations of future yield curve dynamics, as reflected in the traded prices of government securities. These prices incorporate recent developments regarding monetary policy, exchange rate regimes, fiscal policy, and other drivers of economic and financial outcomes. Accordingly, market participants often use a "flat forward" extrapolation in which the forward yield at the LLP maturity is assumed to be the relevant yield for all greater maturities. This approach has the advantage of simplicity, but is tied to just one point on the yield curve. In contrast, a wide variety of yield curve models have been developed to encompass the information in the entire yield curve and develop more robust tools for examining yield curve dynamics and generating yield curve projections. In addition, by introducing a formal model structure, these models can also be used to generate risk measurement and management tools that are critical to the users of these extrapolations.

In this paper, we use the dynamic Nelson-Siegel (DNS) model as developed by Diebold and $\mathrm{Li}$ (2006) to address these various concerns. ${ }^{3}$ The model is structured such that yields are a function of three latent factors and the yield maturity, denoted as $\tau$. The DNS model can easily be used to extrapolate long bond yields beyond the maximum maturity used in model estimation and the market LLP; i.e., for $\tau>L L P$. However, an important concern is whether such extrapolated yields exhibit some sort of bias. If they are systematically too high, the liabilities would be undervalued and likely cause insurance company capital to be set too low to handle their obligations. On the other hand, if the extrapolated yields are too low, the future liabilities would be overvalued, and insurance companies would be penalized by holding too much capital.

In our empirical work, we examine the DNS model's ability to extrapolate long-maturity yields in four countries: Switzerland, Canada, France, and the U.S. In particular, we estimate the DNS model on cross sections of yields with differing maximum maturities - denoted as $\tau_{\max }$ - up to the longest one available within the domestic market; i.e., $\tau_{\max }=L L P$. The differences in long-term yield extrapolations across these estimations provide a measure of their relative accuracy. Our results suggest that such extrapolations are reasonable since the extrapolation errors are relatively small, even with relatively short estimation maturities. For example, our extrapolations for the fifty-year maturity point on the Swiss yield curve using data up to only the fifteen-year maturity have a mean error of about 10 basis points and a root mean-squared error of about 28 basis points. This bias shrinks to 1 basis point and 17 basis points, respectively, when fifty-year projections are based on estimation data up to $\tau_{\max }=30$ years. Similarly, small extrapolation biases are observed for the three other countries. Biases this topic.

${ }^{3}$ This paper complements the analysis by Quaedvlieg and Schotman (2016), who discuss the hedging of the long-term liabilities of pension funds based on the DNS model. Engle et al. (2017) propose an alternative yield curve model to generate long-term bond yields that perform well relative to the DNS model. In addition, Gourieroux and Monfort (2015) review the Smith-Wilson modeling approach adapted by EIOPA for insurance industry regulations. 
of this magnitude are likely tolerable in most calculations for such long-term interest rate risks. $^{4}$

In the analysis, we compare the UFR calculated under the EIOPA rules to the DNS modelimplied UFR. ${ }^{5}$ We find that the DNS model-implied UFR was higher than that implied by the EIOPA rules in the 1995-2007 period. However, since then it has fluctuated around the values used by EIOPA indicating a relative convergence between the two in that market environment.

Given our focus on measuring interest rate risk, we highlight that it is straightforward to simulate the DNS model and perform probability-based stress test exercises, as done in Christensen et al. (2015) with respect to the Fed's own balance sheet as well as Christensen and Lopez (2015) with regard to assessing the severity of banking regulations for interest rate risk. When applied to all four domestic yield curve datasets, our DNS model simulations show that the extrapolation biases are much smaller than the yield changes required to generate stressful outcomes in the tails of the relevant yield curve distributions. For example, based on simulations using the Swiss data, the $90 \%$ confidence band for the ten-year yield easily encompasses changes of \pm 150 basis points, while the extrapolation biases are orders of magnitude smaller.

In summary, the DNS yield curve model provides an established, flexible, and robust framework for generating reliable extrapolations of long-maturity bond yields for financial risk management purposes. With specific regard to insurance companies, this modeling approach should readily accommodate the balance between stability of a key regulatory parameter (i.e., the UFR) and sensitivity to nearer-term economic and financial developments. We acknowledge that there are other methods for modeling, projecting, and assessing interest rate risk in the long run, but our empirical analysis leads us to recommend the DNS modeling approach.

The remainder of the paper is structured as follows. Section 2 introduces the dynamic Nelson-Siegel model we use in the analysis. Section 3 provides a detailed discussion of our modeling results and yield curve extrapolations for Switzerland, while Section 4 contains the corresponding results for Canada, France, and the U.S. Section 5 provides a preliminary comparison of our results with the market-based "flat forward" alternative. Section 6 applies the DNS model to the four domestic yield curves for risk measurement purposes, and Section 7 concludes and reflects on directions for future research.

\footnotetext{
${ }^{4}$ To better gauge the magnitude of these bias estimates, we conduct a detailed simulation study based on the Swiss data; see the details in the online appendix B. The simulation results are that the average of the mean extrapolation error of the fitted 50 -year yields is $-0.39 \%,-0.30 \%$, and $-0.24 \%$, when the maximum yield maturity $\tau_{\max }$ used in the model estimation is 10 years, 20 years, and 30 years, respectively. Comparing this bias with the general variation in yield levels, the DNS model appears to generate reliable extrapolated 50 -year yields, even when $\tau_{\max }=10$ years.

${ }^{5}$ As instantaneous forward rates for maturities in excess of thirty years are practically indistinguishable from the level factor of the DNS model, we set the DNS model-implied UFR equal to the value of this factor.
} 


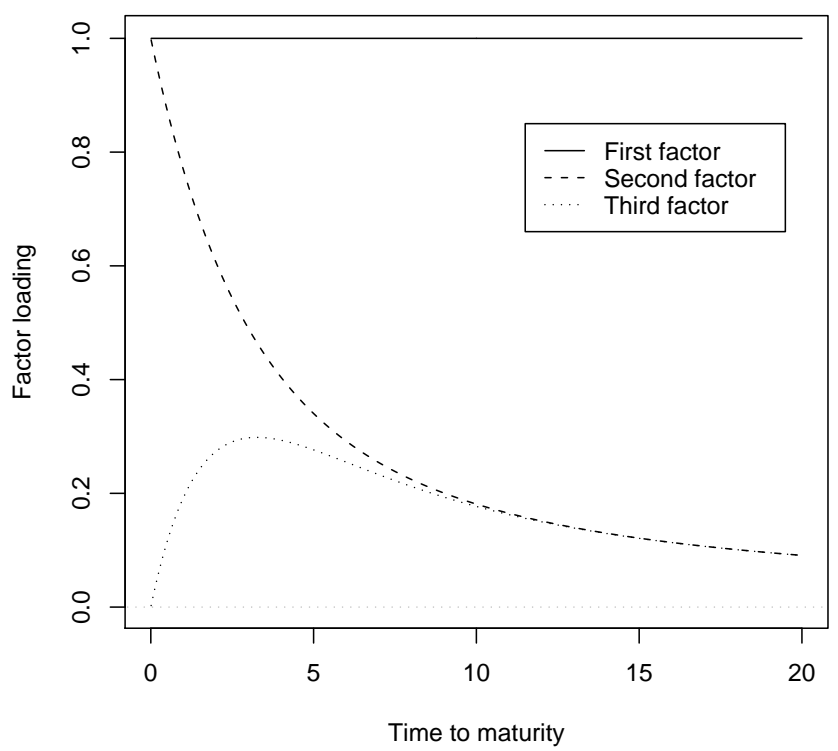

Figure 1: Factor Loadings in the Nelson-Siegel Yield Function

Illustration of the factor loadings on the three state variables in the Nelson-Siegel model. The value for $\lambda$ is 0.55 and maturity is measured in years.

\section{The Dynamic Nelson-Siegel Model}

The Nelson and Siegel (1987) yield curve model assumes that zero-coupon bond yields are functions of the term to maturity $\tau$; specifically,

$$
y(\tau)=\beta_{1}+\beta_{2}\left(\frac{1-e^{-\lambda \tau}}{\lambda \tau}\right)+\beta_{3}\left(\frac{1-e^{-\lambda \tau}}{\lambda \tau}-e^{-\lambda \tau}\right)
$$

where $\beta_{1}, \beta_{2}, \beta_{3}$, and $\lambda$ are parameters. As argued by Diebold and Li (2006), the $\beta$ parameters can be interpreted as level, slope, and curvature factors, respectively, while $\lambda$ determines the maturity at which the peak sensitivity to the curvature factor is located. The loadings on the three factors in the Nelson-Siegel yield curve function are illustrated in Figure 1.

Once the model is viewed as a factor model, we are only a small step away from imposing a dynamic structure on the three $\beta$ factors, as per Diebold et al. (2006). The dynamic version of the model is

$$
y_{t}(\tau)=L_{t}+S_{t}\left(\frac{1-e^{-\lambda \tau}}{\lambda \tau}\right)+C_{t}\left(\frac{1-e^{-\lambda \tau}}{\lambda \tau}-e^{-\lambda \tau}\right)
$$

where the factors $S_{t}, L_{t}$, and $C_{t}$ replace the $\beta$ parameters. The factor dynamics are assumed 
to have the following affine structure:

$$
\left(\begin{array}{c}
L_{t}-\mu_{L} \\
S_{t}-\mu_{S} \\
C_{t}-\mu_{C}
\end{array}\right)=\left(\begin{array}{lll}
a_{11} & a_{12} & a_{13} \\
a_{21} & a_{22} & a_{23} \\
a_{31} & a_{32} & a_{33}
\end{array}\right)\left(\begin{array}{c}
L_{t-1}-\mu_{L} \\
S_{t-1}-\mu_{S} \\
C_{t-1}-\mu_{C}
\end{array}\right)+\left(\begin{array}{c}
\eta_{t}(L) \\
\eta_{t}(S) \\
\eta_{t}(C)
\end{array}\right) .
$$

The error terms are assumed to be Gaussian and to have a full covariance matrix $Q$, which is made to be positive definite by imposing the Choleski decomposition $Q=q q^{\prime}$ with $q$ being a lower triangular matrix. Note that this choice is without loss of generality.

Estimation of the DNS model and related affine term structure models has typically been conducted on zero-coupon yields generated from the underlying government bond prices; such as by using a Svensson (1995) model as done in Gürkaynak et al. (2007) for the U.S. Treasury yield curve. In a recent paper, Andreasen et al. (2019, henceforth ACR) develop an estimation technique based on a "big data" approach using the underlying bond prices directly. They find that this one-step approach is more efficient and reduces estimation noise from the Svensson-type interpolation of yields. In this paper, we use the ACR estimation technique, except for our analysis of the U.S. yield curve in Sections 4.3 and 6.3.

To understand the ACR technique, consider the value at time $t$ of a fixed-coupon bond with maturity $\tau$ that pays a coupon $C$ annually. Its price, denoted $P_{t}^{c}(\tau, C)$, is simply the sum of its cash flow payments weighted by the zero-coupon bond price function $P_{t}(\tau):{ }^{6}$

$$
P_{t}^{c}(\tau, C)=C\left(t_{1}-t\right) P_{t}\left(t_{1}\right)+\sum_{j=2}^{N} C P_{t}\left(t_{j}\right)+P_{t}(\tau), \quad t<t_{1}<\ldots<t_{N}=\tau,
$$

where $P_{t}(\tau)$ is given by $P_{t}(\tau)=\exp \left(-y_{t}(\tau) \tau\right)$, and $y_{t}(\tau)$ is given by equation (1).

Due to the nonlinear relationship between the state variables and the bond prices, the model cannot be estimated with the standard Kalman filter. Instead, we use the extended Kalman filter as in Kim and Singleton (2012). Furthermore, to make the fitted errors comparable across bonds of various maturities, we scale each bond price by its duration, which is calculated before estimation. Thus, the measurement equation for the bond prices in the Kalman filter estimation is of the form:

$$
\frac{P_{t}^{i}\left(\tau^{i}, C^{i}\right)}{D_{t}^{i}\left(\tau^{i}, C^{i}\right)}=\frac{\widehat{P}_{t}^{i}\left(\tau^{i}, C^{i}\right)}{D_{t}^{i}\left(\tau^{i}, C^{i}\right)}+\varepsilon_{t}^{i}
$$

where $\widehat{P}_{t}^{i}\left(\tau^{i}, C^{i}\right)$ is the model-implied price of bond $i$ and $D_{t}^{i}\left(\tau^{i}, C^{i}\right)$ is its duration, which is fixed and calculated before estimation. See ACR for evidence supporting this formulation of the measurement equation. Finally, the error terms in the measurement and transition

\footnotetext{
${ }^{6}$ This is the formula for the clean bond price that does not account for any accrued interest and maps to our bond price data.
} 


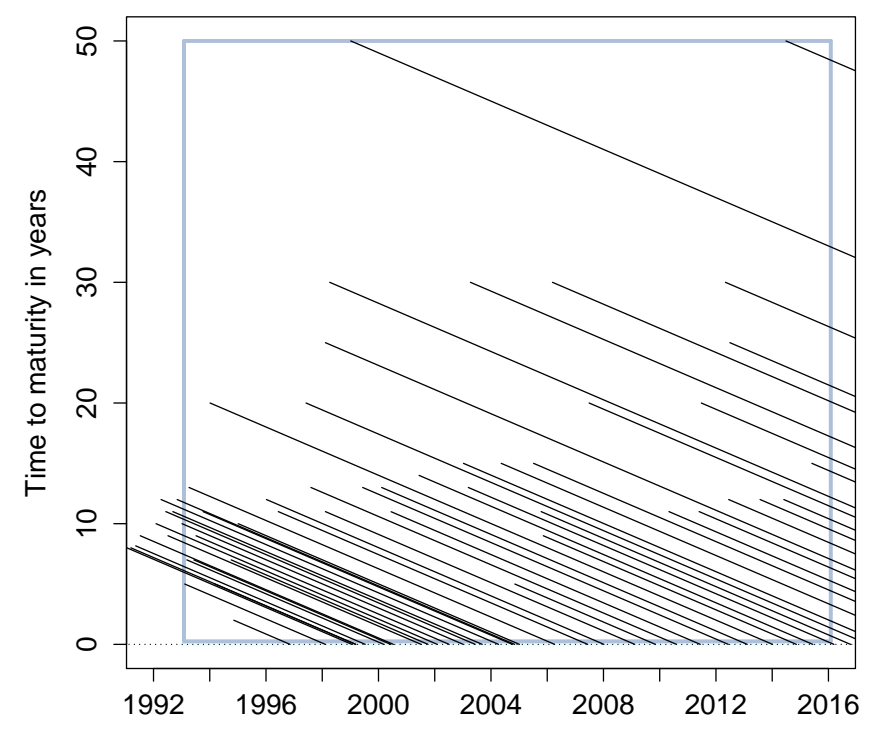

Figure 2: Maturity Distribution of Swiss Confederation Bonds

Illustration of the maturity distribution of the Swiss Confederation bonds considered. The grey rectangle indicates the subsample used throughout the paper and characterized by two sample choices: (1) Due to data quality and availability the sample is limited to the period from January 29, 1993, to January 29, 2016; (2) each bond's price is censored when it has less than three months to maturity to avoid erratic prices close to expiry.

equations are assumed to be independent. Thus, the error structure is given by

$$
\left(\begin{array}{l}
\eta_{t} \\
\varepsilon_{t}
\end{array}\right) \sim N\left[\left(\begin{array}{l}
0 \\
0
\end{array}\right),\left(\begin{array}{cc}
Q & 0 \\
0 & H
\end{array}\right)\right]
$$

where $H=\sigma_{\varepsilon}^{2} I$.

\section{Analysis of Swiss Confederation Bond Data}

In this section, we first describe our sample of Swiss Confederation bonds before we proceed to a description of the DNS model estimation results. We end the section with an analysis of the model fit.

\subsection{Data}

The market for Swiss Confederation bonds is ideal for our analysis since it consists of a sufficient number of bonds to use with the ACR estimation technique and has long-term bond issuance with an LLP of fifty years. Our Swiss bond price data is collected daily at the Swiss 


\begin{tabular}{|c|c|c|}
\hline Confederation bond & $\begin{array}{l}\text { No. } \\
\text { obs. }\end{array}$ & $\begin{array}{c}\text { Issuance } \\
\text { date }\end{array}$ \\
\hline (1) $6.75 \% 1 / 22 / 1999$ & 69 & $1 / 22 / 1991$ \\
\hline (2) $6.25 \% 3 / 15 / 1999$ & 71 & $3 / 15 / 1991$ \\
\hline (3) $6.25 \% 7 / 15 / 1999$ & 74 & $5 / 17 / 1991$ \\
\hline (4) $6.25 \% 7 / 15 / 2000$ & 87 & $7 / 15 / 1991$ \\
\hline (5) $6.5 \% 2 / 5 / 2002$ & 106 & $2 / 5 / 1992$ \\
\hline (6) $6.5 \% 4 / 10 / 2004$ & 132 & 4/10/1992 \\
\hline (7) $6.75 \% 6 / 11 / 2003$ & 122 & 6/11/1992 \\
\hline (8) $7 \% 7 / 9 / 2001$ & 99 & $7 / 9 / 1992$ \\
\hline (9) $7 \% 9 / 10 / 2003$ & 124 & 9/10/1992 \\
\hline (10) $6.25 \% \quad 11 / 5 / 2004$ & 139 & $11 / 5 / 1992$ \\
\hline (11) $6.25 \% 1 / 7 / 2003$ & 117 & $1 / 7 / 1993$ \\
\hline (12) $5.25 \% 2 / 11 / 1998$ & 57 & 2/11/1993 \\
\hline (13) $5 \% 3 / 11 / 2000$ & 81 & $3 / 11 / 1993$ \\
\hline (14) $4.5 \% 4 / 8 / 2006$ & 153 & $4 / 8 / 1993$ \\
\hline (15) $4.5 \% 6 / 10 / 2000$ & 81 & 6/10/1993 \\
\hline (16) $4.5 \% 7 / 8 / 2002$ & 105 & $7 / 8 / 1993$ \\
\hline (17) $4.5 \% \quad 10 / 7 / 2004$ & 129 & $10 / 7 / 1993$ \\
\hline (18) $4.25 \% 1 / 6 / 2014^{*}$ & 237 & $1 / 6 / 1994$ \\
\hline (19) $4 \% 3 / 10 / 1999$ & 56 & $3 / 10 / 1994$ \\
\hline (20) $5.5 \% \quad 10 / 7 / 2001$ & 81 & $10 / 7 / 1994$ \\
\hline (21) $5 \%$ 11/10/1996 & 21 & 11/10/1994 \\
\hline (22) $5.5 \% 1 / 6 / 2005$ & 117 & $1 / 6 / 1995$ \\
\hline (23) $4.25 \% \quad 1 / 8 / 2008$ & 141 & $1 / 8 / 1996$ \\
\hline (24) $4.5 \% 6 / 10 / 2007$ & 129 & 6/10/1996 \\
\hline (25) $4.25 \% 6 / 5 / 2017^{*}$ & 224 & $6 / 5 / 1997$ \\
\hline (26) $3.5 \% 8 / 7 / 2010$ & 153 & $8 / 7 / 1997$ \\
\hline (27) $3.25 \% 2 / 11 / 2009$ & 118 & 2/11/1998 \\
\hline (28) $4 \% 2 / 11 / 2023^{\dagger}$ & 205 & $2 / 11 / 1998$ \\
\hline (29) $4 \% 4 / 8 / 2028^{+}$ & 205 & $4 / 8 / 1998$ \\
\hline (30) $4 \% 1 / 6 / 2049^{\times}$ & 196 & $1 / 6 / 1999$ \\
\hline (31) $2.75 \% 6 / 10 / 2012$ & 149 & 6/10/1999 \\
\hline (32) $4 \% 2 / 11 / 2013$ & 151 & $2 / 11 / 2000$ \\
\hline (33) $4 \%$ 6/10/2011 & 128 & $6 / 13 / 2000$ \\
\hline (34) $3.75 \% 6 / 10 / 2015$ & 164 & $6 / 11 / 2001$ \\
\hline (35) $3 \%$ 1/8/2018 & 154 & $1 / 8 / 2003$ \\
\hline (36) $2.5 \% 3 / 12 / 2016$ & 152 & $3 / 12 / 2003$ \\
\hline (37) $3.5 \% 4 / 8 / 2033^{+}$ & 154 & $4 / 8 / 2003$ \\
\hline (38) $3 \% 5 / 12 / 2019$ & 141 & $5 / 12 / 2004$ \\
\hline (39) $1.75 \% 11 / 5 / 2009$ & 57 & $11 / 5 / 2004$ \\
\hline (40) $2.25 \% 7 / 6 / 2020$ & 127 & $7 / 6 / 2005$ \\
\hline (41) $2 \% 10 / 12 / 2016$ & 123 & $10 / 12 / 2005$ \\
\hline (42) $2 \% 11 / 9 / 2014$ & 105 & $11 / 9 / 2005$ \\
\hline (43) $2.5 \% 3 / 8 / 2036^{+}$ & 119 & $3 / 8 / 2006$ \\
\hline (44) $3.25 \% 6 / 27 / 2027^{*}$ & 104 & $6 / 27 / 2007$ \\
\hline (45) $2 \% 4 / 28 / 2021$ & 70 & $4 / 28 / 2010$ \\
\hline (46) $2 \% 5 / 25 / 2022$ & 57 & $5 / 25 / 2011$ \\
\hline (47) $2.25 \% 6 / 22 / 2031^{*}$ & 56 & $6 / 22 / 2011$ \\
\hline (48) $1.5 \% 4 / 30 / 2042^{+}$ & 45 & $4 / 30 / 2012$ \\
\hline (49) $1.25 \% 6 / 11 / 2024$ & 43 & $6 / 11 / 2012$ \\
\hline (50) $1.25 \% 6 / 27 / 2037^{\dagger}$ & 43 & $6 / 27 / 2012$ \\
\hline (51) $1.5 \% 7 / 24 / 2025$ & 30 & $7 / 24 / 2013$ \\
\hline (52) $1.25 \% 5 / 28 / 2026$ & 20 & $5 / 28 / 2014$ \\
\hline (53) $2 \% 6 / 25 / 2064^{\times}$ & 19 & $6 / 25 / 2014$ \\
\hline (54) $0.5 \% 5 / 27 / 2030$ & 8 & $5 / 27 / 2015$ \\
\hline
\end{tabular}

Table 1: The Universe of Swiss Confederation Bonds

The table reports the characteristics and issuance dates for each Swiss Confederation bond. Also reported are the number of monthly observations for each bond during the sample period from January 29, 1993, to January 29, 2016. Asterisk * indicates twenty-year bonds, dagger $†$ indicates twenty-fiveyear bonds, plus + indicates thirty-year bonds, and cross $\times$ indicates fifty-year bonds (also highlighted in bold). 


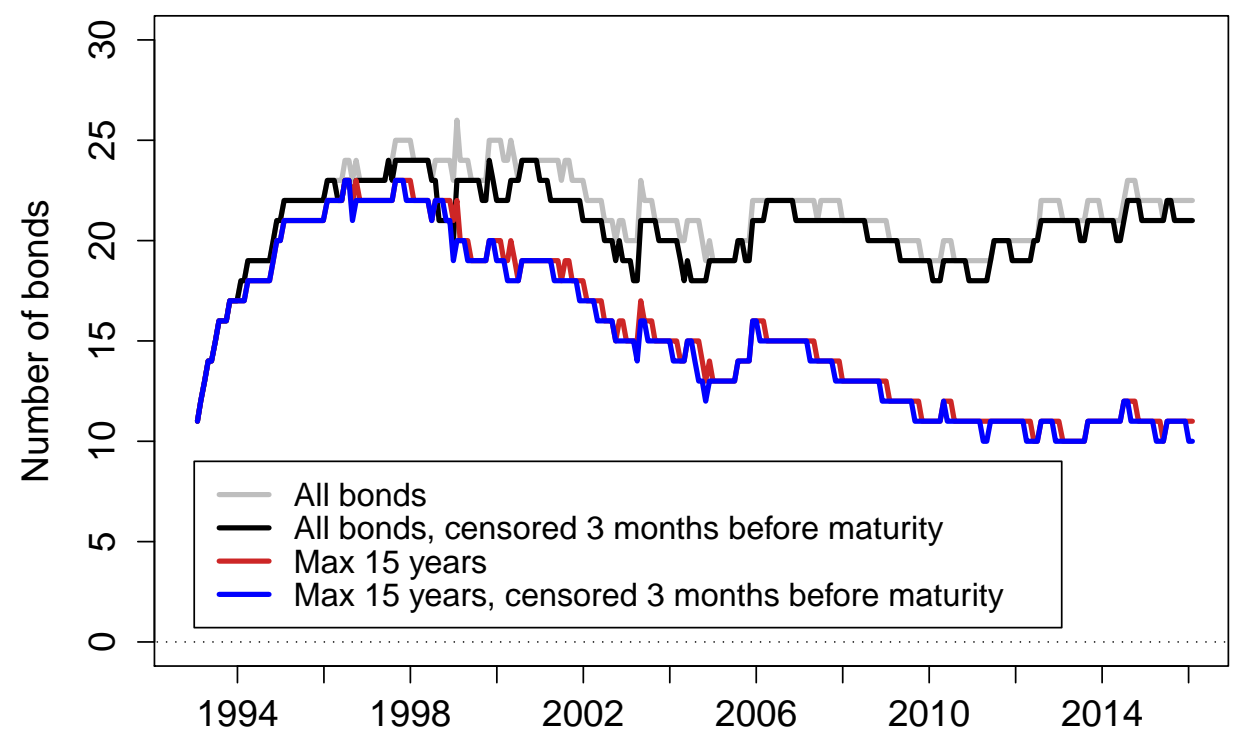

Figure 3: Number of Swiss Confederation Bonds in Sample

Illustration of the number of Swiss Confederation bonds included in the sample at each observation date. The solid grey line refers to the full sample with all bonds included while the solid black line shows the same series, but with each bond censored the last three months before maturity. The solid red line refers to the subset of bonds with at most 15 years to maturity at issuance. The solid blue line indicates the subset of bonds with at most 15 years to maturity at issuance, but with each bond censored the last three months before maturity. The sample is monthly and covers the period from January 29, 1993, to January 29, 2016.

National Bank and available back to the 1980s. However, an inspection of the data reveals that the early part of the sample is characterized by many stale or erratic prices. Thus, we start the monthly estimation sample in January 1993, after the data appear to be systematically reliable across all available bonds. For factor identification in the various models we consider, we need at least five bonds to be trading and accordingly track a few select bonds issued before January 1993 that have systematically reliable prices. These considerations lead us to focus on the universe of 54 Swiss Confederation bonds listed in Table 1. Importantly, the sample contains every Swiss Confederation bond issued since 1993.

Figure 2 shows the maturity distribution of the universe of Swiss Confederation bonds across time from January 1993 to January 2016. The vertical solid grey lines indicate the start and end dates for our sample, while the horizontal solid grey lines indicate the top and bottom of the maturity range considered. The top of the range is fifty years as determined by the longest bond maturity issued by the Swiss Confederation, while the bottom of the range is fixed at three months to avoid erratic prices for bonds approaching maturity. 


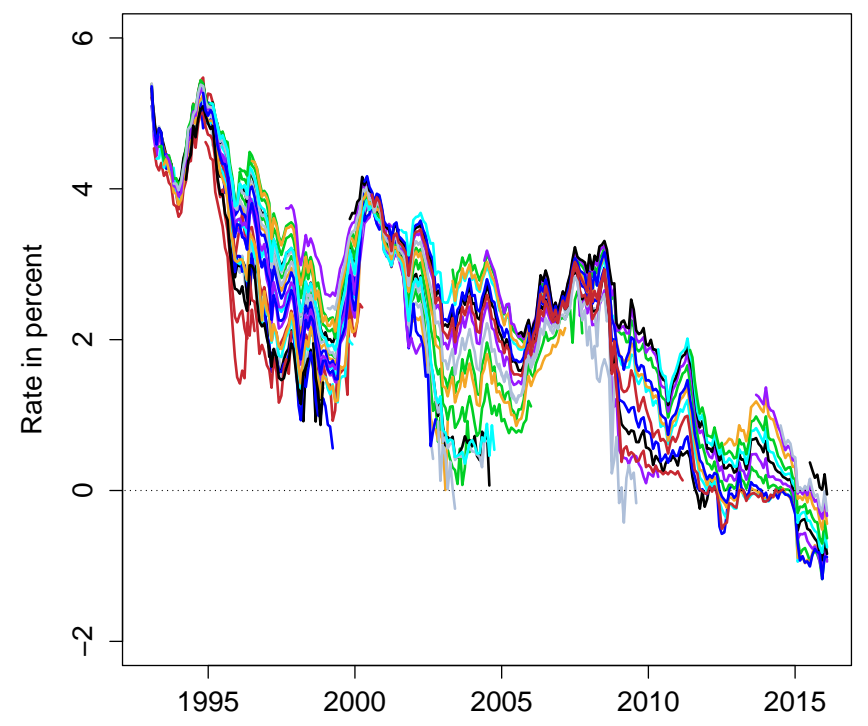

Figure 4: Yield to Maturity of Swiss Confederation Bonds

Illustration of the yield to maturity of the Swiss Confederation bonds considered in this paper, which are subject to two sample choices: (1) sample limited to the period from January 29, 1993, to January 29, 2016; (2) censoring of a bond's price when it has less than three months to maturity.

Figure 3 shows the number of bonds in our sample at each point in time. We note that the total number of outstanding bonds has been fairly stable since the mid-1990s. At the end of our sample period, there were a total of 22 Swiss Confederation bonds outstanding. The figure also shows the available number of bonds when the analysis is restricted to bonds with at most 15 years to maturity at issuance. This maturity limit cuts the number of bonds in half during the post-crisis period, as the Swiss Confederation took advantage of the low interest rate environment and tilted its issuance towards longer-term bonds.

Figure 4 shows the Swiss Confederation bond prices converted into yield to maturity. Note the clear trend towards ever lower yield levels during this 22-year period. Also notable are the negative values for short- and medium-term Swiss yields since 2012. This fact explains why we do not consider term structure models that impose a lower bound for the bond yields, such as Christensen and Rudebusch (2015), as no lower bound appears to yet exist for Swiss yield data. Finally, around the downward trend, there is clear business cycle variation, suggesting that three factors or more are required to capture the yield curve dynamics during the sample period. 


\begin{tabular}{c|cc|cc|cc|cc|cc}
\hline \hline \multirow{2}{*}{ Par. } & \multicolumn{2}{|c|}{ Max 15 years } & \multicolumn{2}{|c|}{ Max 20 years } & \multicolumn{2}{c|}{ Max 25 years } & \multicolumn{2}{c|}{ Max 30 years } & \multicolumn{2}{c}{ Max 50 years } \\
\cline { 2 - 45 } & Est. & SE & Est. & SE & Est. & SE & Est. & SE & Est. & SE \\
\hline$a_{11}$ & 0.9706 & 0.0144 & 0.9842 & 0.0128 & 0.9880 & 0.0122 & 0.9932 & 0.0108 & 0.9973 & 0.0090 \\
$a_{12}$ & -0.0205 & 0.0165 & -0.0064 & 0.0136 & -0.0038 & 0.0134 & 0.0022 & 0.0130 & 0.0043 & 0.0112 \\
$a_{13}$ & 0.0383 & 0.01160 & 0.0275 & 0.0109 & 0.0251 & 0.0109 & 0.0191 & 0.0101 & 0.0162 & 0.0086 \\
$a_{21}$ & -0.0324 & 0.0211 & -0.0174 & 0.0178 & -0.0180 & 0.0171 & -0.0197 & 0.0160 & -0.0185 & 0.0144 \\
$a_{22}$ & 0.9442 & 0.0224 & 0.9710 & 0.0186 & 0.9725 & 0.0180 & 0.9686 & 0.0180 & 0.9746 & 0.0158 \\
$a_{23}$ & 0.0205 & 0.0175 & 0.0037 & 0.0169 & 0.0037 & 0.0171 & 0.0070 & 0.0167 & 0.0026 & 0.0153 \\
$a_{31}$ & 0.1298 & 0.0534 & 0.0880 & 0.0430 & 0.0769 & 0.0400 & 0.0564 & 0.0353 & 0.0435 & 0.0325 \\
$a_{32}$ & 0.1971 & 0.0455 & 0.1359 & 0.0359 & 0.1258 & 0.0337 & 0.1085 & 0.0325 & 0.0935 & 0.0311 \\
$a_{33}$ & 0.8390 & 0.0394 & 0.8656 & 0.0337 & 0.8690 & 0.0316 & 0.8897 & 0.0282 & 0.8982 & 0.0271 \\
\hline$\mu_{1}$ & 0.0271 & 0.0064 & 0.0276 & 0.0069 & 0.0276 & 0.0072 & 0.0273 & 0.0071 & 0.0268 & 0.0075 \\
$\mu_{2}$ & -0.0146 & 0.0059 & -0.0147 & 0.0071 & -0.0145 & 0.0075 & -0.0144 & 0.0072 & -0.0136 & 0.0079 \\
$\mu_{3}$ & -0.0143 & 0.0064 & -0.0122 & 0.0063 & -0.0115 & 0.0062 & -0.0107 & 0.0065 & -0.0091 & 0.0067 \\
\hline$q_{11}$ & 0.0018 & 0.0001 & 0.0017 & 0.0001 & 0.0017 & 0.0001 & 0.0016 & 0.0001 & 0.0015 & 0.0001 \\
$q_{21}$ & -0.0011 & 0.0003 & -0.0011 & 0.0003 & -0.0011 & 0.0003 & -0.0012 & 0.0003 & -0.0010 & 0.0002 \\
$q_{22}$ & 0.0027 & 0.0001 & 0.0025 & 0.0001 & 0.0025 & 0.0001 & 0.0025 & 0.0001 & 0.0025 & 0.0001 \\
$q_{31}$ & -0.0024 & 0.0006 & -0.0019 & 0.0006 & -0.0017 & 0.0005 & -0.0014 & 0.0005 & -0.0011 & 0.0005 \\
$q_{32}$ & -0.0024 & 0.0005 & -0.002 & 0.0005 & -0.0019 & 0.0004 & -0.0019 & 0.0004 & -0.0019 & 0.0004 \\
$q_{33}$ & 0.0049 & 0.0003 & 0.0046 & 0.0003 & 0.0045 & 0.0003 & 0.0044 & 0.0003 & 0.0044 & 0.0003 \\
\hline$\lambda$ & 0.4756 & 0.0118 & 0.3852 & 0.0043 & 0.3702 & 0.0033 & 0.3822 & 0.0026 & 0.365 & 0.0025 \\
\hline$\sigma_{\varepsilon}$ & 0.0009 & $4 \times 10^{-6}$ & 0.0010 & $4 \times 10^{-6}$ & 0.0010 & $4 \times 10^{-6}$ & 0.0010 & $4 \times 10^{-6}$ & 0.0011 & $4 \times 10^{-6}$ \\
\hline \hline
\end{tabular}

Table 2: Estimated Dynamic Parameters for DNS Models of Swiss Bond Prices

The table shows the estimated dynamic parameters for the DNS model of Swiss Confederation fixedcoupon bond prices estimated on samples with varying maximum bond maturity.

\subsection{Estimation Results}

For our yield curve extrapolation, we estimate the DNS model using samples of bond prices with increasing $\tau_{\max }$. In the first estimation, only bonds with 15 years or less to maturity at issuance are included. The maximum maturity is then increased incrementally to 20,25 , and 30 years, before we end with an estimation using all bonds, including the two 50-year bonds; i.e., $\tau_{\max }=L L P$.

The estimated parameters from these five model estimations are reported in Table 2. In general, the level factor is estimated to be very persistent with its AR parameter (i.e., $a_{11}$ ) being very close to a unit root. A similar pattern is observed for the slope factor; i.e., the coefficient $a_{22}$. In contrast, the curvature factor has notably less persistent $a_{33}$ coefficient estimate. As for the off-diagonal parameters in the mean-reversion $A$ matrix, it is mainly the parameters affecting the mean-reversion of the curvature factor (i.e., the coefficients $a_{31}$ and $\left.a_{32}\right)$ that are significant, which is consistent with the findings of Christensen and Krogstrup (2019), although they only consider Swiss Confederation bond yields with maturities up to ten years.

Figure 5 shows the three estimated state variables from all five model estimations. All three factors remain qualitatively similar across the model estimations. Hence, they appear to have only modest sensitivity to the choice of the maximum bond maturity. The most 


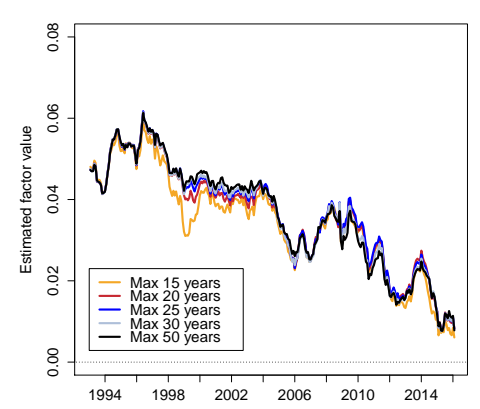

(a) $L_{t}$

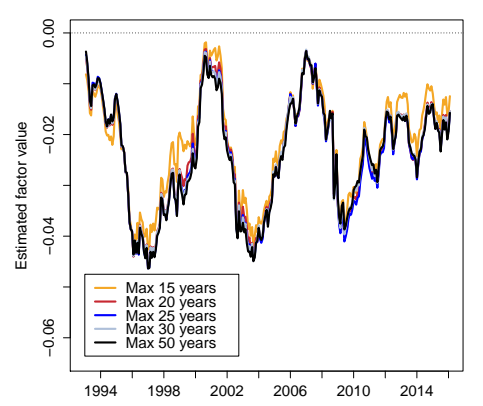

(b) $S_{t}$

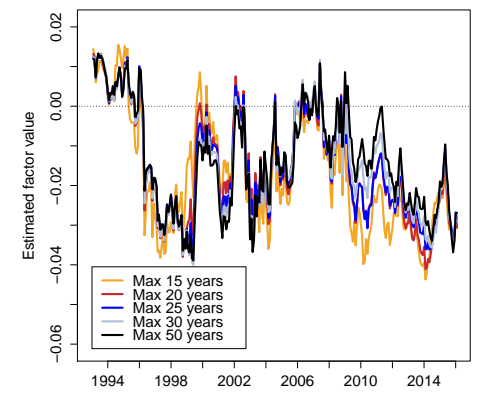

(c) $C_{t}$

Figure 5: Estimated State Variables

Illustration of the estimated level, slope, and curvature state variables from the DNS model estimated on samples with varying maximum bond maturity. The data are monthly covering the period from January 29, 1993, to January 29, 2016.

notable variation is observed in Figure 5(a), which compares the estimated level factors. The estimation using at most 15-year bonds delivers somewhat lower values for $L_{t}$ during the 1998-2002 period. Beyond that it is challenging to detect any material systematic differences regarding the filtered state variables. This observation is consistent with the minor differences in the estimated model parameters discussed above.

\subsection{Analysis of Model Fit}

Table 3 evaluates the ability of the DNS model to match the market prices of the coupon bonds. The pricing errors are here computed based on the implied yield on each coupon bond to make these errors comparable across securities. That is, for the price on the $i$ th coupon bond $P_{t}^{i}\left(\tau, C^{i}\right)$, we find the value of $y_{t}^{i, c}$ that solves

$$
P_{t}^{i}\left(\tau^{i}, C^{i}\right)=C^{i}\left(t_{1}-t\right) \exp \left\{-y_{t}^{i, c}\left(t_{1}-t\right)\right\}+\sum_{j=2}^{N} C^{i} \exp \left\{-y_{t}^{i, c}\left(t_{j}-t\right)\right\}+\exp \left\{-y_{t}^{i, c}\left(t_{N}-t\right)\right\}
$$

For the model-implied estimate of this bond price, denoted $\hat{P}_{t}^{i}\left(\tau, C^{i}\right)$, we find the corresponding implied yield $\hat{y}_{t}^{i, c}$ and report the pricing error as $y_{t}^{i, c}-\hat{y}_{t}^{i, c}$.

Table 3 reports the summary statistics for the fit to all bonds in the sample from the five model estimations. We stress that for the estimations with $\tau_{\max }<50$ years, the statistics for the bonds not included in their respective estimations measure the model's ability to extrapolate, rather than to fit, the prices of those securities. These results are highlighted with grey shading. At the bottom of the table, the statistics for all errors combined are reported where we see the expected pattern that it is easier to fit yields in-sample than out-of-sample as the error decreases as more and more long bonds are included in the estimation sample. Indeed, all out-of-sample extrapolated error cells in the table are statically significantly different at 


\begin{tabular}{|c|c|c|c|c|c|c|c|c|c|c|c|}
\hline \multirow{2}{*}{$\begin{array}{c}\text { Maturity } \\
\text { bucket }\end{array}$} & \multirow{2}{*}{$\begin{array}{l}\text { No. } \\
\text { obs. }\end{array}$} & \multicolumn{2}{|c|}{ Max 15 years } & \multicolumn{2}{|c|}{ Max 20 years } & \multicolumn{2}{|c|}{ Max 25 years } & \multicolumn{2}{|c|}{ Max 30 years } & \multicolumn{2}{|c|}{ Max 50 years } \\
\hline & & Mean & RMSE & Mean & RMSE & Mean & RMSE & Mean & RMSE & Mean & RMSE \\
\hline $0-2$ & 716 & -0.55 & 14.00 & -0.80 & 14.60 & -0.84 & 14.81 & -0.68 & 14.96 & -0.56 & 15.31 \\
\hline $2-4$ & 848 & 1.35 & 8.18 & 0.96 & 8.70 & 1.00 & 9.01 & 0.91 & 9.38 & 0.67 & 9.76 \\
\hline $4-6$ & 857 & -0.69 & 7.76 & -0.44 & 7.74 & -0.37 & 7.77 & -0.71 & 7.96 & -0.78 & 8.15 \\
\hline $6-8$ & 767 & 0.11 & 5.81 & 0.15 & 6.16 & 0.09 & 6.20 & -0.21 & 6.32 & -0.14 & 6.34 \\
\hline $8-10$ & 694 & 0.24 & 4.98 & -0.67 & 6.05 & -0.95 & 6.34 & -1.07 & 6.82 & -0.90 & 6.92 \\
\hline $10-12$ & 507 & 1.55 & 6.10 & -0.10 & 6.08 & -0.62 & 6.47 & -0.53 & 7.02 & -0.44 & 7.42 \\
\hline $12-14$ & 266 & 4.03 & 6.73 & 1.12 & 4.70 & 0.36 & 4.78 & 0.66 & 6.10 & 0.26 & 7.28 \\
\hline $14-16$ & 169 & 9.11 & 14.64 & 3.57 & 7.03 & 2.36 & 6.35 & 2.27 & 7.08 & 2.06 & 8.21 \\
\hline $16-18$ & 154 & 5.67 & $13.10^{* *}$ & 0.99 & 6.86 & -0.23 & 5.95 & 2.44 & 4.73 & 2.78 & 6.34 \\
\hline $18-20$ & 168 & 9.34 & $19.40^{* *}$ & 3.00 & 9.96 & 1.69 & 8.55 & 3.46 & 7.81 & 4.93 & 8.95 \\
\hline $20-22$ & 103 & 9.84 & $16.77^{* *}$ & 3.67 & $10.89^{* *}$ & 2.35 & 9.15 & 3.00 & 6.75 & 2.45 & 6.19 \\
\hline $22-24$ & 120 & 6.72 & $25.74^{* *}$ & -2.83 & $17.44^{* *}$ & -4.12 & 14.47 & -1.22 & 8.88 & -0.91 & 7.29 \\
\hline $24-26$ & 84 & -0.46 & $22.49^{* *}$ & -5.21 & $18.62^{* *}$ & -7.93 & 18.30 & -4.14 & 11.49 & -2.42 & 9.08 \\
\hline $26-28$ & 94 & 4.08 & $21.05^{* *}$ & -3.05 & $17.11^{* *}$ & -5.36 & $16.95^{* *}$ & -3.66 & 11.86 & -4.81 & 9.09 \\
\hline $28-30$ & 87 & 9.01 & $32.52^{* *}$ & -2.88 & $22.34^{* *}$ & -4.85 & $17.23^{* *}$ & -3.65 & 13.16 & -4.29 & 12.18 \\
\hline $30<$ & 215 & 9.65 & $28.32^{* *}$ & 0.79 & $22.91^{* *}$ & -1.54 & $21.93^{* *}$ & 1.12 & $17.49^{* *}$ & 0.63 & 12.33 \\
\hline All bonds & 5,849 & 1.93 & 12.44 & 0.05 & 10.47 & -0.41 & 10.17 & -0.10 & 9.50 & -0.09 & 9.37 \\
\hline
\end{tabular}

Table 3: Summary Statistics of Fitted Errors of Swiss Confederation Bond Yields This table reports the mean pricing errors (Mean) and the root mean-squared pricing errors (RMSE) of the Swiss bond prices for the DNS model estimated on samples with varying maximum bond maturity. The pricing errors are reported in basis points and computed as the difference between the implied yield on the coupon bond and the model-implied yield on this bond. For each set of estimation results grey shading indicates the part that represent out-of-sample extrapolated errors rather than in-sample fitted errors. For the out-of-sample extrapolated errors, asterisks * and ** indicate significant difference at the 5 percent and 1 percent levels, respectively, relative to the corresponding in-sample fitted error series from the estimation reported in the last two columns, which uses all available data. The data are monthly and cover the period from January 29, 1993, to January 29, 2016.

the one percent level relative to the estimation that includes all bond prices reported in the last two columns of the table. ${ }^{7}$ Still, it is the case that even when the model is estimated with $\tau_{\max }=15$ years, it still gives a satisfactory overall fit to the data with a mean error of 2 basis points and a root mean-squared error (RMSE) of 12 basis points. Notably, the out-of-sample RMSE for a given maturity bucket steadily declines as more bonds are included in the estimation sample; for example, the out-of-sample RMSE for the last maturity bucket declines steadily from 28.32 basis points with $\tau_{\max }=15$ to 17.49 basis points with $\tau_{\max }=30$. If we focus narrowly on the fit of the two 50-year bonds reported in the greater than thirty-year maturity bucket, we see a fairly consistent pattern of improved fit as the maximum bond maturity in the model estimation is increased. With all bonds included, the model is able to fit these two bonds with almost no bias on average and a RMSE of 12 basis points.

To give a sense of the variation across time in the model fit, Figure 6 shows the time series of the fitted errors of the 54 individual bonds from the estimation with all bonds included.

\footnotetext{
${ }^{7}$ We use a standard F-test of differences in the variances of the extrapolated error series and the matching full sample error series with the null hypothesis being no difference.
} 


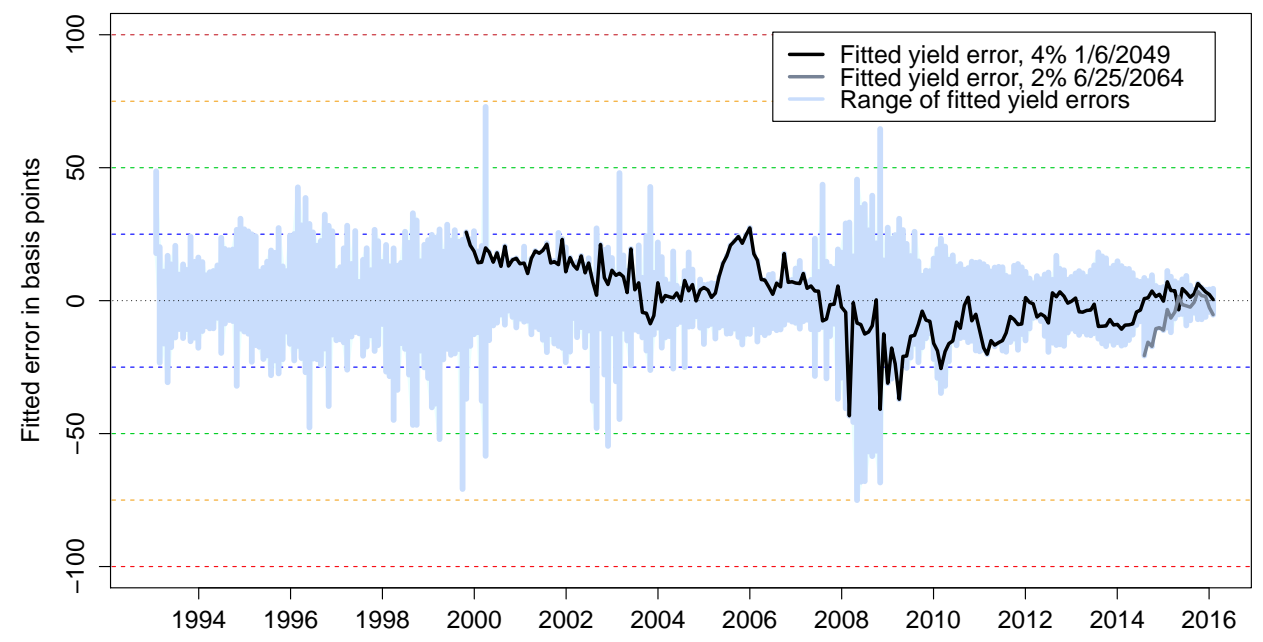

Figure 6: Fitted Errors from the DNS Model of Swiss Confederation Bond Prices Illustration of the fitted errors of Swiss Confederation bond yields to maturity implied by the DNS model estimated with all Swiss Confederation bonds. The data are monthly and cover the period from January 29, 1993, to January 29, 2016.

Two things stand out. First, there are brief periods such as the peak of the financial crisis during which the model faces challenges in fitting the data. Second, since 2010 with few exceptions, the model has been able to price all bonds with an error of less than 20 basis points. As parts of the Swiss Confederation bond yield curve have been in negative territory since 2012, this demonstrates that the DNS model continues to deliver very accurate fit even in negative yield environments.

\section{Other Domestic Yield Curve Extrapolations}

In this section, we repeat the analysis above using Canadian, French, and U.S. government bond prices. We find similar yield curve dynamics and model performance across all four countries, which is supportive evidence in favor of the DNS model for cross-country analysis of this type.

\subsection{Analysis of Canadian Government Bond Prices}

The available universe of individual Canadian government fixed-coupon bonds since January 2000 is illustrated in Figure 7 and is identical to the sample analyzed in ACR. ${ }^{8}$ These bonds are all marketable, non-callable bonds denominated in Canadian dollars that pay a fixed rate

\footnotetext{
${ }^{8}$ The full list of Canadian government securities considered is available in the online appendix.
} 


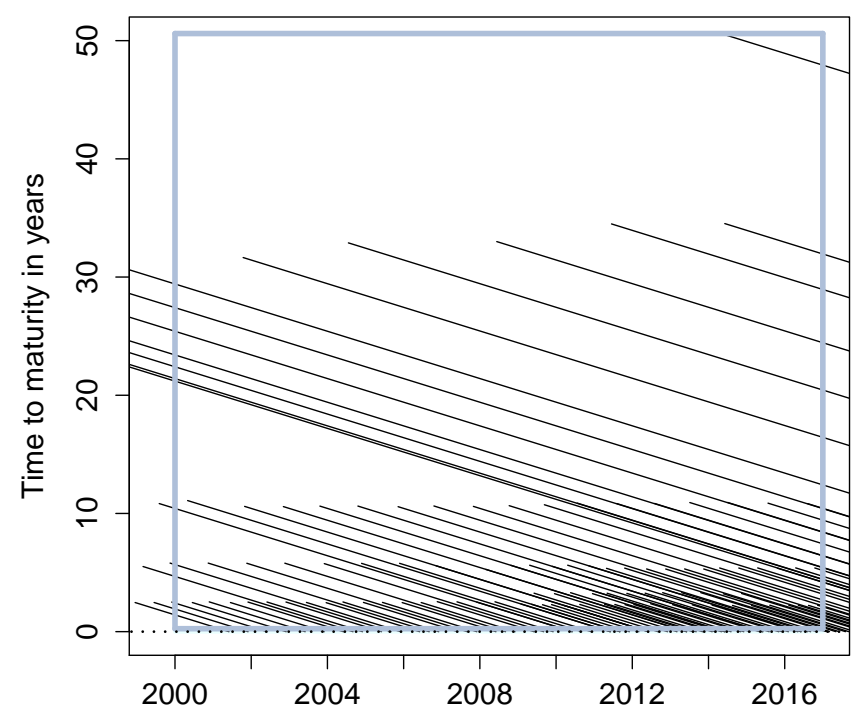

Figure 7: Maturity Distribution of Canadian Government Bonds

Illustration of the maturity distribution of the Canadian government fixed-coupon bonds considered. The grey rectangle indicates the subsample used in the estimation, which is characterized by two sample choices: (1) sample limited to the period from January 31, 2000, to April 29, 2016; (2) censoring of the price of each bond when it has less than three months to maturity.

of interest semi-annually. ${ }^{9}$ We note that the Bank of Canada systematically has been issuing two-, five-, ten-, and thirty-year fixed-coupon bonds during this period. In addition, it has repeatedly issued three-year bonds since 2008. For our analysis, the key thing to note is that a single fifty-year bond was issued in April 2014. This issuance pattern suggests examining three samples: a sample of bonds with ten years or less to maturity, another sample of bonds with thirty years or less to maturity, and the full sample.

Figure 8 shows the time series of the yields to maturity implied by the observed bond prices. First, we note the downward trend of the general yield level since 2000. The ten-year yield dropped from above 6 percent to below 2 percent over the shown period. Second, as for other domestic yields, there is clear business cycle variation in the shape of the yield curve around this lower trend.

The estimated parameters from the three model estimations are reported in Table 4. We note that the level and curvature factors tend to become more persistent as we increase the maximum bond maturity used in model estimation, while the slope factor exhibits the opposite pattern and becomes less persistent. This difference in pattern relative to the Swiss results may be explained by the estimated $\lambda$ parameter, which increases as we increase the maximum

\footnotetext{
${ }^{9}$ This information is available at http://www.fin.gc.ca/invest/instru-eng.asp
} 


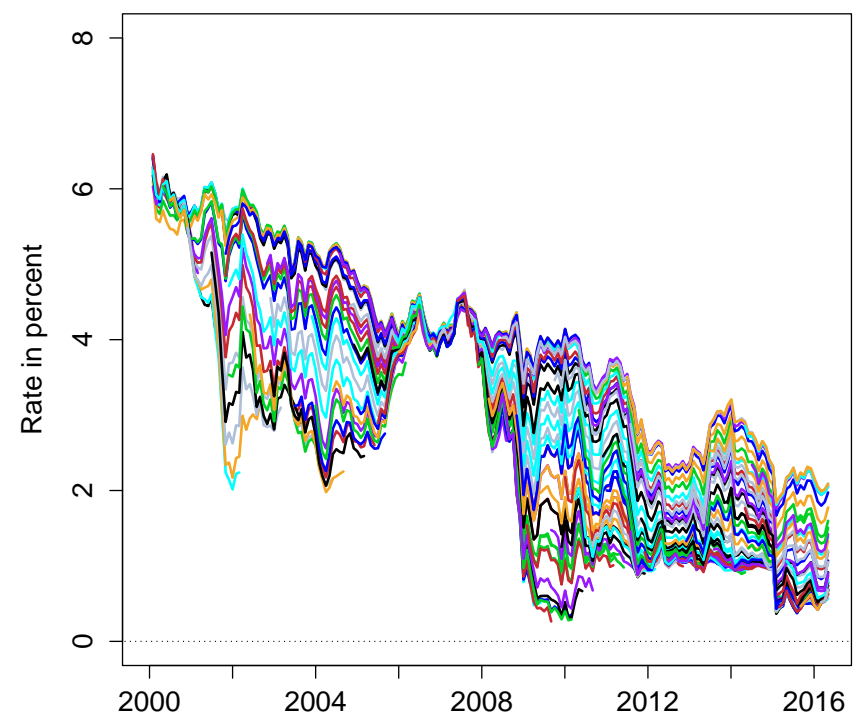

Figure 8: Yield to Maturity of Canadian Government Bonds

Illustration of the yield to maturity of the Canadian government fixed-coupon bonds considered in the paper, which are subject to two sample choices: (1) sample limited to the period from January 31, 2000, to April 29, 2016; (2) censoring of the price of each bond when it has less than three months to maturity.

bond maturity. This implies that the factor loading on the slope factor decays faster when we include all bonds. Focusing on the off-diagonal parameters in the mean-reversion $A$ matrix, we note that it is mainly the parameters affecting the mean-reversion of the curvature factor, i.e., the coefficients $a_{21}$ and $a_{23}$, that are significant, which is consistent with the findings of Christensen et al. (2017). Finally, there is some tendency for the volatility of all three factors to decline as the maximum maturity increases.

Figure 9 shows the three estimated state variables from all three Canadian DNS model estimations; i.e., $\tau_{\max } \in[10,30,50]$ years. In general, all three factors remain qualitatively similar across the model estimations. Hence, they appear to have only modest sensitivity to the choice of $\tau_{\max }$.

Summary statistics for the fitted errors of the individual bonds are reported in Table 5. Focusing on the fit of long-term bonds with more than thirty years to maturity, which includes the fit of the fifty-year bond issued in 2014, we note that the DNS model's ability to fit those very long-term bond yields does improve as we increase the maximum bond maturity. However, importantly, the mean fitted error for this category of bond yields is practically the same negative 15 basis points across all three model estimations. Thus, the average fitted error for these bonds is not very sensitive to the maximum maturity used in model estimation, although we stress that we still find statistically significant differences in the variances of the 


\begin{tabular}{c|cc|cc|cc}
\hline \hline \multirow{2}{*}{ Par. } & \multicolumn{2}{|c|}{ Max 10 years } & \multicolumn{2}{c|}{ Max 30 years } & \multicolumn{2}{c}{ Max 50 years } \\
\cline { 2 - 7 } & Est. & SE & Est. & SE & Est. & SE \\
\hline$a_{11}$ & 0.9721 & 0.0182 & 1.0040 & 0.0130 & 1.0056 & 0.0126 \\
$a_{12}$ & 0.0126 & 0.0171 & 0.0216 & 0.0105 & 0.0222 & 0.0107 \\
$a_{13}$ & 0.0186 & 0.0132 & -0.0045 & 0.0093 & -0.0051 & 0.0093 \\
$a_{21}$ & -0.0215 & 0.0282 & -0.0880 & 0.0267 & -0.0889 & 0.0265 \\
$a_{22}$ & 0.9601 & 0.0230 & 0.9389 & 0.0206 & 0.9365 & 0.0207 \\
$a_{23}$ & 0.0279 & 0.0201 & 0.0779 & 0.0206 & 0.0798 & 0.0207 \\
$a_{31}$ & 0.0925 & 0.0629 & 0.0800 & 0.0550 & 0.0733 & 0.0548 \\
$a_{32}$ & 0.0016 & 0.0407 & -0.0049 & 0.0336 & -0.0045 & 0.0338 \\
$a_{33}$ & 0.8848 & 0.0400 & 0.9078 & 0.0379 & 0.9100 & 0.0378 \\
\hline$\mu_{1}$ & 0.0443 & 0.0106 & 0.0492 & 0.0024 & 0.0492 & 0.0024 \\
$\mu_{2}$ & -0.0216 & 0.0112 & -0.0210 & 0.0065 & -0.0207 & 0.0066 \\
$\mu_{3}$ & -0.0150 & 0.0106 & -0.0126 & 0.0035 & -0.0128 & 0.0035 \\
\hline$q_{11}$ & 0.0026 & 0.0002 & 0.0016 & 0.0001 & 0.0016 & 0.0001 \\
$q_{21}$ & -0.0028 & 0.0002 & -0.0020 & 0.0002 & -0.0020 & 0.0002 \\
$q_{22}$ & 0.0019 & 0.0001 & 0.0019 & 0.0001 & 0.0019 & 0.0001 \\
$q_{31}$ & -0.0035 & 0.0005 & 0.0000 & 0.0006 & 0.0000 & 0.0006 \\
$q_{32}$ & 0.0006 & 0.0006 & 0.0011 & 0.0005 & 0.0011 & 0.0005 \\
$q_{33}$ & 0.0064 & 0.0003 & 0.0058 & 0.0003 & 0.0058 & 0.0003 \\
\hline$\lambda$ & 0.4353 & 0.0023 & 0.4913 & 0.0015 & 0.5013 & 0.0016 \\
\hline$\sigma_{\varepsilon}$ & 0.0004 & $2 \times 10^{-6}$ & 0.0008 & $2 \times 10^{-6}$ & 0.0008 & $2 \times 10^{-6}$ \\
\hline \hline
\end{tabular}

Table 4: Estimated Dynamic Parameters for DNS Models of Canadian Bond Prices The table shows the estimated dynamic parameters for the DNS model of Canadian government fixedcoupon bond prices estimated on samples with varying maximum bond maturity.

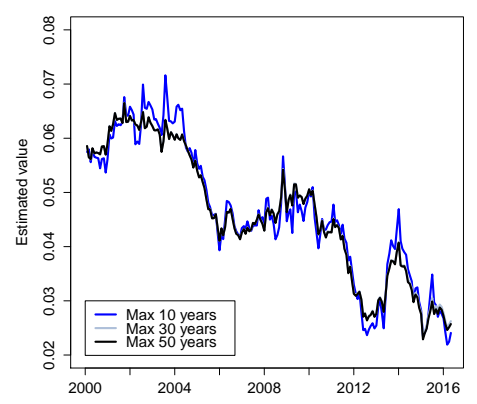

(a) $L_{t}$

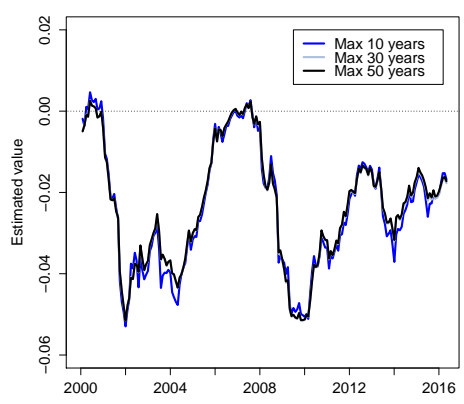

(b) $S_{t}$

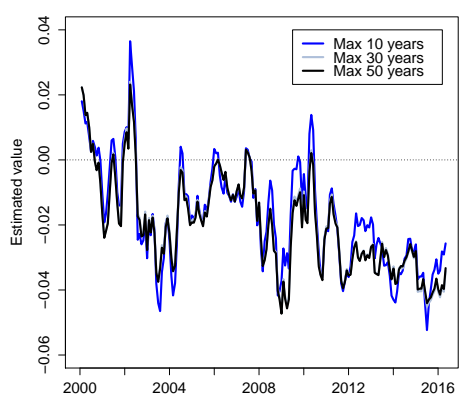

(c) $C_{t}$

Figure 9: Estimated State Variables of Canadian DNS Models

Illustration of the estimated level, slope, and curvature state variables from the DNS model estimated on samples of Canadian government fixed-coupon bond prices with varying maximum bond maturity. The data are monthly covering the period from January 31, 2000, to April 29, 2016.

extrapolated errors from the estimation using only prices of bonds with at most ten years to maturity at issuance relative to the estimation using all bond prices. 


\begin{tabular}{c|c|cc|cc|cc}
\hline \hline Maturity & No. & \multicolumn{2}{|c|}{ Max 10 years } & \multicolumn{2}{c|}{ Max 30 years } & \multicolumn{2}{c}{ Max 50 years } \\
\cline { 3 - 8 } bucket & obs. & Mean & RMSE & Mean & RMSE & Mean & RMSE \\
\hline $0-2$ & 1,472 & -0.03 & 4.85 & 0.21 & 5.88 & 0.23 & 5.86 \\
$2-4$ & 1,098 & 0.18 & 3.32 & 0.26 & 4.27 & 0.31 & 4.26 \\
$4-6$ & 744 & 0.21 & 3.64 & -1.92 & 4.48 & -2.08 & 4.60 \\
$6-8$ & 404 & 0.39 & 3.12 & -2.56 & 6.09 & -2.76 & 6.21 \\
$8-10$ & 477 & 0.92 & 3.33 & -2.17 & 5.89 & -2.28 & 5.94 \\
$10-12$ & 289 & 4.36 & $9.00^{* *}$ & 0.54 & 6.10 & 0.55 & 6.12 \\
$12-14$ & 155 & 12.28 & $16.30^{* *}$ & 5.98 & 7.73 & 6.09 & 7.80 \\
$14-16$ & 168 & 5.90 & $11.77^{* *}$ & 3.51 & 5.30 & 3.61 & 5.40 \\
$16-18$ & 179 & 3.98 & $14.49^{* *}$ & 3.29 & 5.77 & 3.44 & 6.01 \\
$18-20$ & 192 & 0.99 & $12.57^{* *}$ & 3.59 & 5.41 & 3.75 & 5.57 \\
$20-22$ & 186 & 7.87 & $15.58^{* *}$ & 5.00 & 6.91 & 5.21 & 7.14 \\
$22-24$ & 142 & -1.56 & $12.09^{* *}$ & 0.56 & 5.61 & 0.82 & 5.73 \\
$24-26$ & 124 & 0.93 & $17.10^{* *}$ & -1.05 & 6.24 & -0.66 & 6.34 \\
$26-28$ & 113 & -10.17 & $16.31^{* *}$ & -9.47 & 12.45 & -9.08 & 12.39 \\
$28-30$ & 94 & -8.90 & $20.53^{* *}$ & -12.50 & 15.90 & -12.11 & 15.70 \\
$30<$ & 194 & -15.64 & $22.37^{* *}$ & -16.44 & 19.04 & -15.18 & 18.11 \\
\hline All bonds & 6,031 & 0.38 & 9.02 & -0.75 & 6.86 & -0.68 & 6.81 \\
\hline \hline
\end{tabular}

Table 5: Summary Statistics of Fitted Errors of Canadian Bond Yields

This table reports the mean pricing errors (Mean) and the root mean-squared pricing errors (RMSE) of the Canadian bond prices for the DNS model estimated on samples with varying maximum bond maturity. The pricing errors are reported in basis points and computed as the difference between the implied yield on the coupon bond and the model-implied yield on this bond. For each set of estimation results grey shading indicates the part that represent out-of-sample extrapolated errors rather than insample fitted errors. For the out-of-sample extrapolated errors, asterisks $*$ and $* *$ indicate significant difference at the 5 percent and 1 percent levels, respectively, relative to the corresponding in-sample fitted error series from the estimation reported in the last two columns, which uses all available data. The data are monthly and cover the period from January 31, 2000, to April 29, 2016.

\subsection{Analysis of French Government Bond Prices}

The available universe of individual French government fixed-coupon bonds since January 1999 is illustrated in Figure 10. ${ }^{10}$ These bonds are all marketable, non-callable bonds denominated in euros that pay a fixed rate of interest annually. We note that the France Trésor systematically has been issuing two-, five-, ten-, fifteen-, and thirty-year fixed-coupon bonds during this period. For our analysis, the key thing to note is that a total of three fifty-year bonds have been issued since 2005. This issuance pattern invites a split of the data into four maturity samples: bonds with ten years or less to maturity, bonds with fifteen years or less to maturity, those with thirty years or less to maturity, and the full sample.

Figure 11 shows the time series of the yields to maturity implied by the observed French bond prices. First, we note the downward trend of the general yield level since 1999. The tenyear yield dropped from around 4.5 percent to close to zero over the shown period. Second, we observe clear business cycle variation in the shape of the yield curve around this lower trend. Finally, regarding the important question of a lower bound, the European Central Bank has

\footnotetext{
${ }^{10}$ The full list of French government securities considered is available in the online appendix.
} 


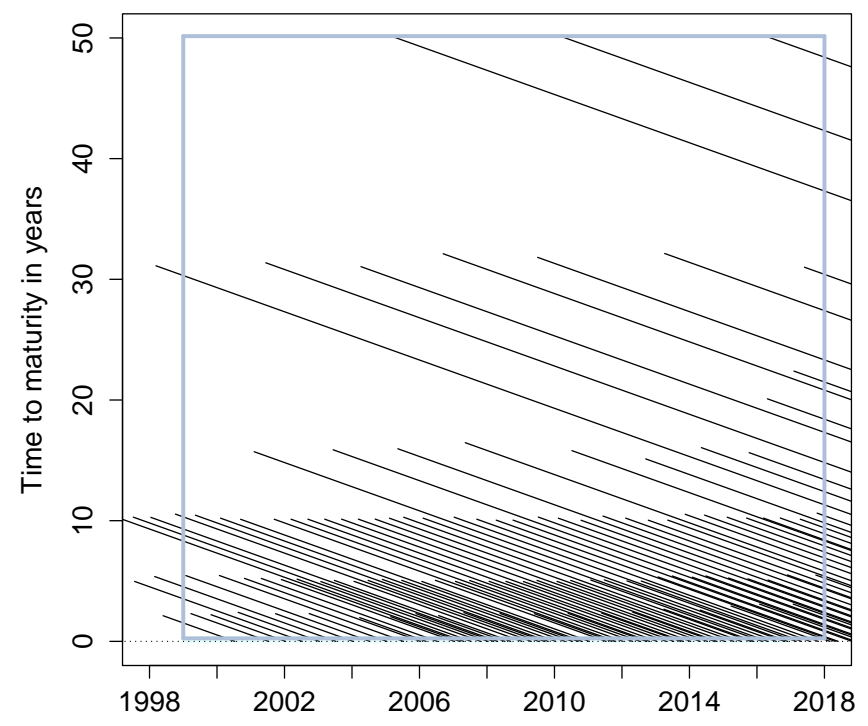

Figure 10: Maturity Distribution of French Government Bonds

Illustration of the maturity distribution of the French government fixed-coupon bonds considered in the paper. The grey rectangle indicates the subsample used throughout the paper and characterized by two sample choices: (1) sample limited to the period from January 29, 1999, to December 29, 2017; (2) censoring of the price of each bond when it has less than three months to maturity.

lowered its conventional policy rate deep into negative territory and engaged in large-scale asset purchases - also known as quantitative easing - since January 2015. As a consequence, short- and medium-term French bond yields are significantly negative towards the end of our sample. Thus, it is not clear that one would need to impose a lower bound to model this data.

The estimated parameters from the four model estimations are reported in Table 6 . We note that the level and slope factors tend to become less persistent as we increase the maximum bond maturity used in model estimation, while the curvature factor exhibits the opposite pattern and becomes more persistent. Similar to the Canadian data this pattern may be tied to the estimated $\lambda$ parameter, which increases as we increase the maximum bond maturity. This implies that the factor loading on the slope factor decays faster when we include all bonds. Focusing on the off-diagonal parameters in the mean-reversion $A$ matrix, we note that it is mainly the parameters that determine the interactions between the level and slope factors, i.e., the coefficients $a_{12}$ and $a_{21}$, that are significant. Finally, there is some tendency for the volatility of all three factors to decline as the maximum maturity increases similar to what we observed for the Swiss and Canadian data.

Figure 12 shows the three estimated state variables from all four French DNS model estimations. In general, the pattern is that all three factors remain qualitatively similar 


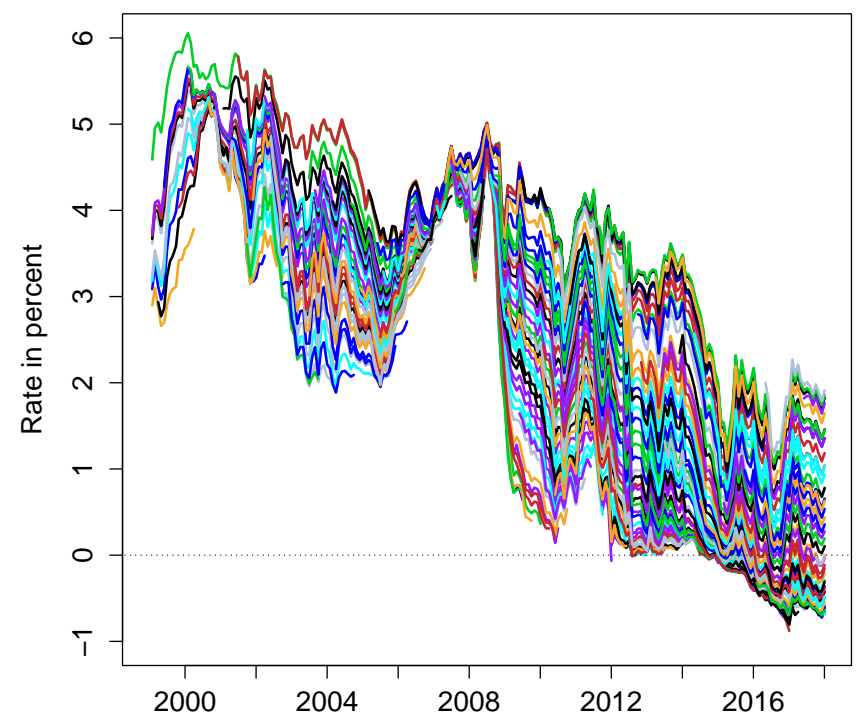

Figure 11: Yield to Maturity of French Government Bonds

Illustration of the yield to maturity of the French government fixed-coupon bonds considered in the paper, which are subject to two sample choices: (1) sample limited to the period from January 29, 1999, to December 29, 2017; (2) censoring of the price of each bond when it has less than three months to maturity.

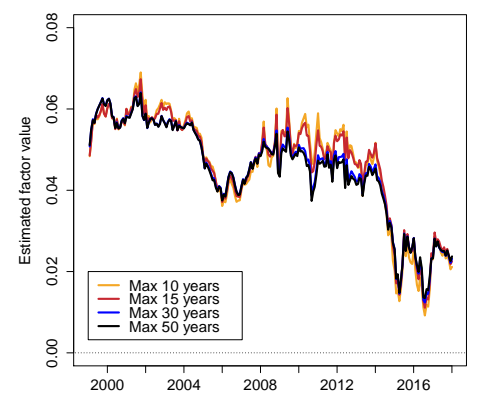

(a) $L_{t}$

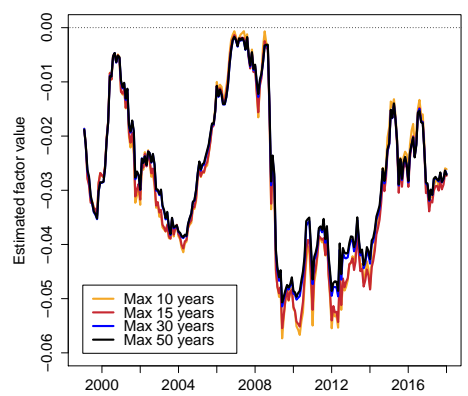

(b) $S_{t}$

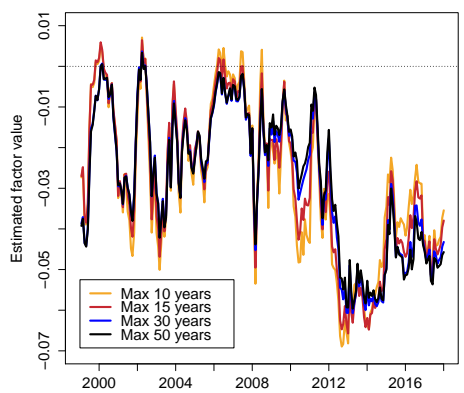

(c) $C_{t}$

Figure 12: Estimated State Variables of French DNS Models

Illustration of the estimated level, slope, and curvature state variables from the DNS model estimated on samples of French government fixed-coupon bond prices with varying maximum bond maturity. The data are monthly covering the period from January 29, 1999, to December 29, 2017.

across all model estimations. Hence, they appear to have only modest sensitivity to the choice of the maximum bond maturity. In short, it is challenging to detect any material systematic differences regarding either the estimated model parameters or the filtered state variables. 


\begin{tabular}{c|cc|cc|cc|cc}
\hline \hline \multirow{2}{*}{ Par. } & \multicolumn{2}{|c|}{ Max 10 years } & \multicolumn{2}{c|}{ Max 15 years } & \multicolumn{2}{c|}{ Max 30 years } & \multicolumn{2}{c}{ Max 50 years } \\
\cline { 2 - 9 } & Est. & SE & Est. & SE & Est. & SE & Est. & SE \\
\hline$a_{11}$ & 0.9791 & 0.0181 & 0.9722 & 0.0171 & 0.9595 & 0.0170 & 0.9211 & 0.0176 \\
$a_{12}$ & 0.0118 & 0.0208 & 0.0147 & 0.0181 & 0.0217 & 0.0136 & 0.0289 & 0.0123 \\
$a_{13}$ & 0.0272 & 0.0170 & 0.0218 & 0.0156 & 0.0144 & 0.0126 & 0.0147 & 0.0117 \\
$a_{21}$ & -0.0031 & 0.0254 & 0.0011 & 0.0266 & 0.0153 & 0.0265 & 0.0568 & 0.0262 \\
$a_{22}$ & 0.9631 & 0.0234 & 0.9627 & 0.0214 & 0.9611 & 0.0174 & 0.9542 & 0.0161 \\
$a_{23}$ & 0.0056 & 0.0206 & 0.0091 & 0.0196 & 0.0133 & 0.0179 & 0.0125 & 0.0167 \\
$a_{31}$ & 0.0349 & 0.0494 & 0.0426 & 0.0472 & 0.0599 & 0.0424 & 0.0883 & 0.0480 \\
$a_{32}$ & 0.0329 & 0.0407 & 0.0229 & 0.0364 & 0.0065 & 0.0306 & -0.0062 & 0.0293 \\
$a_{33}$ & 0.9100 & 0.0378 & 0.9246 & 0.0358 & 0.9319 & 0.0337 & 0.9346 & 0.0330 \\
\hline$\mu_{1}$ & 0.0468 & 0.0143 & 0.0479 & 0.0132 & 0.0441 & 0.0068 & 0.0472 & 0.0092 \\
$\mu_{2}$ & -0.0254 & 0.0098 & -0.0277 & 0.0095 & -0.0311 & 0.0102 & -0.0313 & 0.0174 \\
$\mu_{3}$ & -0.0229 & 0.0124 & -0.0234 & 0.0145 & -0.0285 & 0.0103 & -0.0252 & 0.0162 \\
\hline$q_{11}$ & 0.0030 & 0.0002 & 0.0027 & 0.0001 & 0.0023 & 0.0001 & 0.0024 & 0.0001 \\
$q_{21}$ & -0.0032 & 0.0002 & -0.0030 & 0.0002 & -0.0025 & 0.0002 & -0.0026 & 0.0002 \\
$q_{22}$ & 0.0018 & 0.0001 & 0.0018 & 0.0001 & 0.0019 & 0.0001 & 0.0019 & 0.0001 \\
$q_{31}$ & -0.0038 & 0.0006 & -0.0027 & 0.0006 & -0.0013 & 0.0005 & -0.0012 & 0.0005 \\
$q_{32}$ & -0.0002 & 0.0006 & -0.0001 & 0.0005 & -0.0001 & 0.0005 & -0.0002 & 0.0005 \\
$q_{33}$ & 0.0060 & 0.0003 & 0.0058 & 0.0003 & 0.0054 & 0.0003 & 0.0053 & 0.0003 \\
\hline$\lambda$ & 0.4012 & 0.0011 & 0.3937 & 0.0006 & 0.4261 & 0.0005 & 0.4319 & 0.0005 \\
\hline$\sigma_{\varepsilon}$ & 0.0004 & $1 \times 10^{-6}$ & 0.0004 & $1 \times 10^{-6}$ & 0.0006 & $2 \times 10^{-6}$ & 0.0007 & $2 \times 10^{-6}$ \\
\hline \hline
\end{tabular}

Table 6: Estimated Dynamic Parameters for DNS Models of French Bond Prices The table shows the estimated dynamic parameters for the DNS model of French government fixedcoupon bond prices estimated on samples with varying maximum bond maturity.

Table 7 reports the summary statistics of the fitted errors of the French bond prices. In this case, we do see a quite notable improvement in the DNS model's ability to fit bonds with maturities longer than thirty years. When $\tau_{\max }=10$ years, the RMSE of these bonds is 34 basis points, which is reduced to 28,13 , and 10 basis points, respectively, when the maximum maturity is increased to 15, 30, and 50 years, respectively. Thus, as long as the DNS model is estimated using bonds with maturities up to thirty years, the extrapolated fit is only modestly less accurate than the fit obtained with all bonds included in the model estimation. ${ }^{11}$ Overall, this again confirms the findings reported earlier for Swiss and Canadian government bond data regarding the accuracy of the DNS model in extrapolating long bond yields.

\subsection{Analysis of U.S. Treasury Bond Yields}

As noted earlier, our analysis of the U.S. Treasury yield curve is not based on individual bonds. Instead, the specific Treasury yields used are zero-coupon yields constructed by the method described in Gürkaynak at al. (2007). The analysis conducted here is to provide a reference point for the other countries examined before. The Treasury yields used have the following 11 maturities: three-month, six-month, one-year, two-year, three-year, five-year,

\footnotetext{
${ }^{11}$ Note that it remains the case that the extrapolated error series highlighted in grey are mostly significantly different from the corresponding fitted errors from the full sample estimation in terms of variance.
} 


\begin{tabular}{c|c|cc|cc|cc|cc}
\hline \hline Maturity & No. & \multicolumn{2}{|c|}{ Max 10 years } & \multicolumn{2}{c|}{ Max 15 years } & \multicolumn{2}{c|}{ Max 30 years } & \multicolumn{2}{c}{ Max 50 years } \\
\cline { 3 - 10 } bucket & obs. & Mean & RMSE & Mean & RMSE & Mean & RMSE & Mean & RMSE \\
\hline $0-2$ & 1,504 & -1.04 & 6.05 & -1.10 & 6.44 & -0.98 & 7.27 & -0.91 & 7.53 \\
$2-4$ & 1,532 & 2.03 & 4.62 & 1.97 & 4.97 & 1.91 & 6.09 & 1.73 & 6.49 \\
$4-6$ & 1,196 & 3.57 & 5.92 & 3.59 & 5.84 & 3.03 & 5.69 & 2.83 & 5.65 \\
$6-8$ & 812 & 3.65 & 6.23 & 3.72 & 6.85 & 3.49 & 7.65 & 3.51 & 7.77 \\
$8-10$ & 885 & 5.86 & 7.23 & 5.81 & 7.54 & 6.19 & 9.22 & 6.44 & 9.76 \\
$10-12$ & 223 & 6.52 & $8.82^{*}$ & 5.50 & 6.41 & 6.34 & 8.51 & 6.83 & 9.64 \\
$12-14$ & 196 & 5.91 & $10.92^{* *}$ & 4.40 & 6.61 & 6.26 & 7.96 & 6.86 & 9.41 \\
$14-16$ & 208 & 7.38 & $13.28^{* *}$ & 5.41 & 8.17 & 7.53 & 9.12 & 8.31 & 10.54 \\
$16-18$ & 63 & 8.19 & $15.48^{* *}$ & 4.56 & 8.43 & 7.18 & 9.41 & 8.08 & 11.81 \\
$18-20$ & 92 & 3.16 & $16.35^{* *}$ & 0.07 & 9.17 & 5.29 & 7.50 & 6.15 & 9.72 \\
$20-22$ & 95 & 2.85 & $18.10^{* *}$ & 0.45 & $12.07^{* *}$ & 6.63 & 9.01 & 8.49 & 11.23 \\
$22-24$ & 110 & 1.50 & $21.96^{* *}$ & -0.63 & $14.70^{* *}$ & 5.22 & 7.09 & 6.96 & 8.60 \\
$24-26$ & 120 & 1.61 & $21.95^{* *}$ & -0.33 & $15.97^{* *}$ & 5.72 & 8.61 & 7.32 & 9.03 \\
$26-28$ & 128 & -2.82 & $23.81^{* *}$ & -3.48 & $18.04^{* *}$ & 4.09 & 9.00 & 6.20 & 8.59 \\
$28-30$ & 144 & -2.02 & $27.82^{* *}$ & -2.22 & $22.53^{* *}$ & 3.76 & 11.64 & 5.52 & 10.56 \\
$30<$ & 383 & -7.63 & $34.26^{* *}$ & -10.77 & $27.54^{* *}$ & -2.79 & $13.36^{* *}$ & -0.76 & 9.94 \\
\hline All bonds & 7,691 & 2.08 & 11.96 & 1.62 & 9.85 & 2.66 & 7.86 & 2.95 & 8.01 \\
\hline \hline
\end{tabular}

Table 7: Summary Statistics of Fitted Errors of French Bond Yields

This table reports the mean pricing errors (Mean) and the root mean-squared pricing errors (RMSE) of the French bond prices for the DNS model estimated on samples with varying maximum bond maturity. The pricing errors are reported in basis points and computed as the difference between the implied yield on the coupon bond and the model-implied yield on this bond. For each set of estimation results grey shading indicates the part that represent out-of-sample extrapolated errors rather than insample fitted errors. For the out-of-sample extrapolated errors, asterisks $*$ and $* *$ indicate significant difference at the 5 percent and 1 percent levels, respectively, relative to the corresponding in-sample fitted error series from the estimation reported in the last two columns, which uses all available data. The data are monthly and cover the period from January 29, 1999, to December 29, 2017.

seven-year, ten-year, fifteen-year, twenty-year, and thirty-year. ${ }^{12}$ The sample is limited to monthly observations over the period from January 31, 1995, to December 31, 2016. Figure 13 illustrates the constructed time series of the three-month, two-year, five-year, ten-year, and thirty-year Treasury zero-coupon yields. Although short- and medium-term U.S. Treasury yields were constrained at times by the zero lower bound during the period from December 2008 to December 2015 when the Fed kept its target overnight federal funds rate in the range between 0 and $0.25 \%$, Christensen and Rudebusch (2016) only report fairly modest gains from using a shadow-rate modeling approach to account for the asymmetric yield behavior during this period. Based on this evidence, we choose to proceed with the Gaussian DNS model even for this data, which also has the benefit that our U.S. results will be comparable to those reported for the other countries. As before, the U.S. yield sample invites a natural split into three subsamples that we will consider in our analysis: yields with ten years or less to maturity, yields with twenty years to maturity or less, and the full sample of yields.

\footnotetext{
${ }^{12}$ The Board of Governors in Washington D.C. updates the factors and parameters of this method daily, see also the related website http://www.federalreserve.gov/pubs/feds/2006/index.html
} 


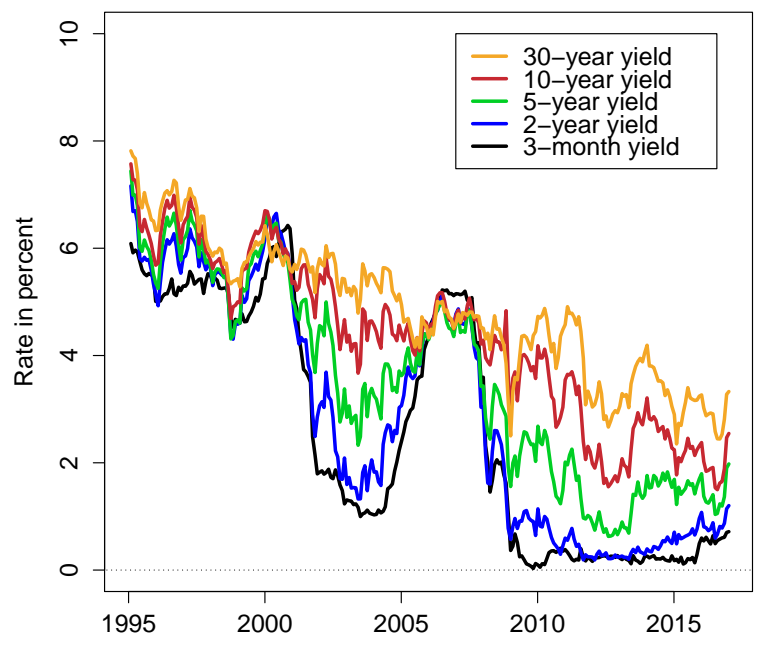

Figure 13: Time Series of U.S. Treasury Bond Yields

Illustration of the monthly U.S. Treasury zero-coupon bond yields covering the period from January 31, 1995, to December 31, 2016. The yields shown have maturities: three-month, two-year, five-year, ten-year and thirty-year.

\begin{tabular}{c|cc|cc|cc}
\hline \hline \multirow{2}{*}{ Par. } & \multicolumn{2}{|c|}{ Max 10 years } & \multicolumn{2}{c|}{ Max 20 years } & \multicolumn{2}{c}{ Max 30 years } \\
\cline { 2 - 7 } & Est. & SE & Est. & SE & Est. & SE \\
\hline$a_{11}$ & 0.9719 & 0.0194 & 0.9861 & 0.0158 & 0.9906 & 0.0144 \\
$a_{12}$ & 0.0181 & 0.0251 & 0.0227 & 0.0176 & 0.0228 & 0.0163 \\
$a_{13}$ & 0.0027 & 0.0156 & -0.0071 & 0.0119 & -0.0088 & 0.0107 \\
$a_{21}$ & -0.0213 & 0.0245 & -0.0238 & 0.0236 & -0.0335 & 0.0239 \\
$a_{22}$ & 0.9277 & 0.0255 & 0.9365 & 0.0195 & 0.9313 & 0.0189 \\
$a_{23}$ & 0.0532 & 0.0169 & 0.0553 & 0.0148 & 0.0589 & 0.0150 \\
$a_{31}$ & 0.0302 & 0.0729 & 0.0270 & 0.0588 & 0.0261 & 0.0572 \\
$a_{32}$ & 0.0308 & 0.0402 & 0.0315 & 0.0324 & 0.0370 & 0.0321 \\
$a_{33}$ & 0.9343 & 0.0295 & 0.9362 & 0.0291 & 0.9433 & 0.0304 \\
\hline$\mu_{1}$ & 0.0540 & 0.0077 & 0.0552 & 0.0097 & 0.0544 & 0.0107 \\
$\mu_{2}$ & -0.0231 & 0.0173 & -0.0211 & 0.0164 & -0.0183 & 0.0174 \\
$\mu_{3}$ & -0.0187 & 0.0202 & -0.0183 & 0.0185 & -0.0163 & 0.0226 \\
\hline$q_{11}$ & 0.0031 & 0.0001 & 0.0025 & 0.0001 & 0.0023 & 0.0001 \\
$q_{21}$ & -0.0032 & 0.0002 & -0.0027 & 0.0002 & -0.0025 & 0.0002 \\
$q_{22}$ & 0.0017 & 0.0001 & 0.0018 & 0.0001 & 0.0018 & 0.0001 \\
$q_{31}$ & -0.0021 & 0.0004 & 0.0002 & 0.0004 & 0.0015 & 0.0004 \\
$q_{32}$ & -0.0010 & 0.0005 & -0.0002 & 0.0005 & -0.0001 & 0.0005 \\
$q_{33}$ & 0.0080 & 0.0003 & 0.0072 & 0.0004 & 0.0063 & 0.0004 \\
\hline$\lambda$ & 0.4964 & 0.0039 & 0.4944 & 0.0022 & 0.5586 & 0.0044 \\
\hline$\sigma_{\varepsilon}$ & 0.0006 & $6 \times 10^{-6}$ & 0.0008 & $6 \times 10^{-6}$ & 0.0013 & $6 \times 10^{-6}$ \\
\hline \hline
\end{tabular}

Table 8: Estimated Dynamic Parameters for DNS Models of U.S. Treasury Yields The table shows the estimated dynamic parameters for the DNS model of U.S. Treasury yields estimated on samples with varying maximum bond maturity. 


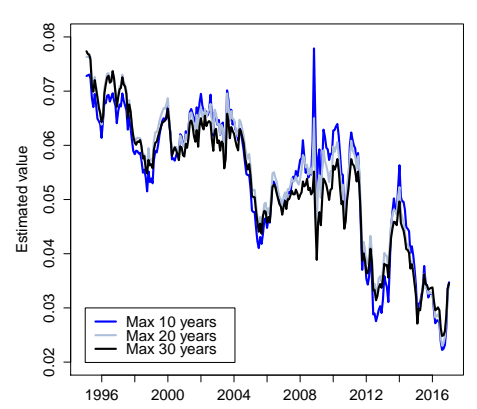

(a) $L_{t}$

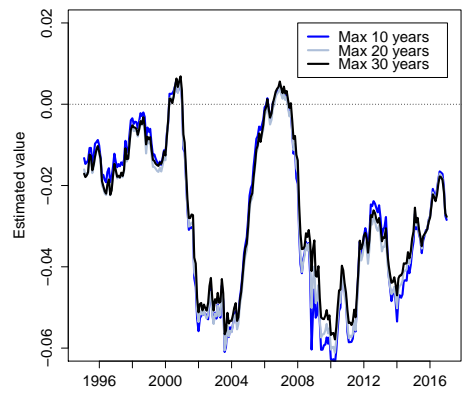

(b) $S_{t}$

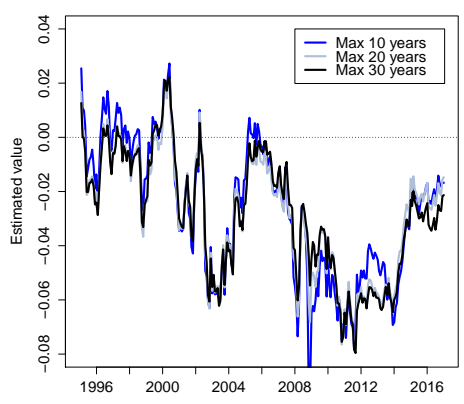

(c) $C_{t}$

Figure 14: Estimated State Variables of U.S. DNS Models

Illustration of the estimated level, slope, and curvature state variables from the DNS model estimated on samples of U.S. Treasury yields with varying maximum yield maturity. The data are monthly covering the period from January 31, 1995, to December 31, 2016.

A few observations regarding the estimated dynamic parameters reported in Table 8 are worth noting. First, there is a tendency for the level factor to become more persistent as the maximum yield maturity used in model estimation increases. Also, the overall estimated volatility of the state variables exhibit modest declines as the maximum yield maturity goes up. Second, in terms of the off-diagonal parameters in the $A$ matrix, it appears that only $a_{21}$ and $a_{23}$ have some significance, while the other off-diagonal parameters are entirely insignificant. These findings are consistent with the U.S. Treasury yield model preferred by Christensen and Rudebusch (2012), although they consider a different sample period and use the arbitrage-free Nelson-Siegel models developed in Christensen et al. (2011).

Figure 14 shows the three estimated state variables from all three U.S. DNS model estimations. In general, the pattern is that all three factors remain qualitatively similar across all model estimations. Hence, we only observe modest sensitivity to the choice of the maximum bond maturity. Similarly, the parameter estimates in Table 8 do not vary much across the different estimation samples. A likely explanation is that the algorithms used in generating these yields smooth out a large degree of idiosyncratic bond variation, which likely contributes to much less data variation in this data set as we introduce longer maturity bonds into the estimation.

Table 9 presents the fitted errors across the estimations. As expected, the RMSE measure for $\tau_{\max }=30$ years decreases as the sample maturity is extended. Notably, the mean error does not improve much from the $\tau_{\max }=10$ to the $\tau_{\max }=20$ samples, although the RMSE does decline and statistically significantly so. Focusing on the LLP 30-year yield, the mean error of -17 basis points for the shortest maturity sample is reasonably close to the -12 basis point mean error observed in-sample for the longest maturity sample. This result suggests that the DNS extrapolation error in the U.S. Treasury data is a bit larger than observed for the other national datasets; however, it remains relatively small relative to a full-sample 


\begin{tabular}{|c|c|c||c|c||c|c|}
\hline Maturity & \multicolumn{2}{|c||}{ Max 10 years } & \multicolumn{2}{c||}{ Max 20 years } & \multicolumn{2}{c|}{ Max 30 years } \\
\cline { 2 - 7 } in months & Mean & RMSE & Mean & RMSE & Mean & RMSE \\
\hline 3 & -0.71 & 5.84 & -1.37 & 7.71 & -1.65 & 7.59 \\
6 & -0.46 & 2.63 & -0.67 & 2.97 & -0.32 & 4.03 \\
12 & 0.81 & 6.27 & 1.24 & 7.75 & 2.01 & 8.38 \\
24 & 1.69 & 3.75 & 2.62 & 7.52 & 2.56 & 8.44 \\
36 & 0.45 & 2.54 & 1.26 & 4.65 & -0.05 & 6.45 \\
60 & -2.21 & 5.35 & -2.40 & 5.88 & -4.70 & 7.74 \\
84 & -1.87 & 3.50 & -3.23 & 8.29 & -4.53 & 9.84 \\
120 & 2.25 & 5.00 & -0.56 & 7.85 & 1.00 & 11.77 \\
180 & 7.48 & $18.37^{* *}$ & 3.17 & 5.08 & 8.93 & 14.15 \\
240 & 4.90 & $28.19^{* *}$ & -0.23 & 9.44 & 8.13 & 11.00 \\
360 & -16.94 & $48.72^{* *}$ & -22.91 & $37.78^{* *}$ & -11.82 & 22.37 \\
\hline All yields & -0.42 & 18.27 & -2.10 & 13.19 & -0.04 & 11.18 \\
\hline
\end{tabular}

Table 9: Summary Statistics of Fitted Errors of U.S. Treasury Yields

The table reports the mean and root mean squared fitted errors from the DNS model estimated on samples of U.S. Treasury yields with varying maximum yield maturity. For each set of estimation results grey shading indicates the part that represent out-of-sample extrapolated errors rather than in-sample fitted errors. For the out-of-sample extrapolated errors, asterisks * and ** indicate significant difference at the 5 percent and 1 percent levels, respectively, relative to the corresponding in-sample fitted error series from the estimation reported in the last two columns, which uses all available data. The data are monthly covering the period from January 31, 1995, to December 31, 2016.

estimation and thus useful for extrapolation purposes.

\section{Comparisons with Existing Alternatives}

In this section, we first compare yield extrapolations from the DNS model to a conventional approach frequently used by financial market participants known as "flat forwards." This work leads us to define the DNS model-implied ultimate forward rate by the value of the DNS level factor, which we then compare to the UFRs used in the EIOPA rules.

\subsection{The Ultimate Forward Rate from DNS Models}

Market participants have been known to price illiquid longer-term bonds using so-called "flat forward" rate curves. In practice, given a forward rate curve estimated from bond prices with a maximum maturity $\tau_{L L P}$, forward rates for longer maturities are simply set equal to the forward rate at $\tau_{L L P}$. Formally, the extrapolated forward rate curve is given by

$$
f(\tau)= \begin{cases}\widehat{f}(\tau) & \text { for } \tau \leq \tau_{L L P} \\ \widehat{f}\left(\tau_{L L P}\right) & \text { for } \tau>\tau_{L L P}\end{cases}
$$

where $\widehat{f}(\tau)$ represents fitted forward rates.

For the Swiss dataset, Figure 15 shows the fitted and extrapolated forward rates as of 


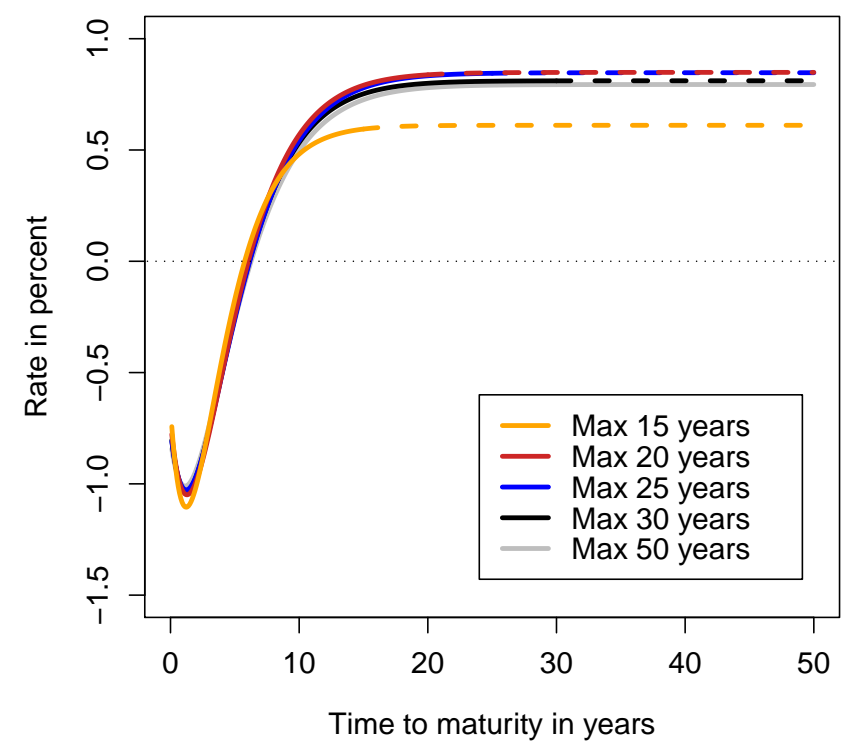

Figure 15: Fitted and Extrapolated Forward Rate Curves from the DNS Model Illustration of the fitted and extrapolated forward rate curves implied by the independent-factors DNS model as of January 29, 2016, when estimated on samples with varying maximum bond maturity. The in-sample fitted part of each forward curve is shown with a solid line, while the out-of-sample extrapolated part of each forward curve is shown with a dashed line.

January 29, 2016, as implied by the DNS model with the five $\tau_{\max }$ values reported in Table 3. First, note the similarity in the forward rates for maturities up to ten years, which is due to the fact that all the estimations are fitting the same data in this maturity range equally well. Second and more importantly for our analysis, we observe that most extrapolations are very similar to the fitted forward rate curve using all the available data; i.e., the estimates for $\tau_{\max }=50$ years. The exception is the estimation with $\tau_{\max }=15$ years, which generates extrapolated yields that are more than 25 basis points below the observed longer-term yields.

Thus, a yield curve extrapolation technique based on the DNS model is consistent with the "flat forward" approach. Combined with the small extrapolated errors for the two 50year bonds reported in Table 3 for the four estimations where these bonds are excluded, our empirical evidence suggests that the DNS model extrapolation approach is roughly equivalent to the "flat forwards" approach. However, the modeling approach provides us with a richer framework for risk management purposes (i.e., moving beyond just the first moments of the data used for standard pricing purposes), which we explore in Section $6{ }^{13}$ For now we use

\footnotetext{
${ }^{13}$ Note that this result may suggest that investors price these long bonds using flat forwards. In theory, this would imply that the bonds are mispriced. However, in practice it is not possible to short those long bonds and go long shorter bonds without assuming some roll-over risk, and once that is taken into consideration there may not be exploitable mispricing in the Swiss Confederation bond market. Alternatively, the underpricing of the long bonds could reflect liquidity premiums that investors demand as compensation for potentially facing
} 
this evidence to define the DNS model-implied ultimate forward rate by the value of the DNS level factor since instantaneous forward rates for maturities in excess of thirty years are practically indistinguishable from the value of this factor within the DNS model.

\subsection{The Ultimate Forward Rate in the EIOPA Framework}

The purpose of this section is to compare the ultimate forward rate (UFR) prescribed in the EIOPA framework for the purpose of discounting long-dated liability cash flows to the asymptotic rate calculated from the DNS model (i.e., the level factor).

The yield used to discounting liabilities beyond the last liquid point (LLP) is the result of an interpolation between the yield at the LLP and the UFR, which is reached at the maximum of LLP +40 years and 60 years. The details of this methodology can be found in EIOPA (2018b).

The UFR calculation is detailed in EIOPA (2017c) and is summarized in the following. The first step is to calculate the annual real rate for each year since 1961. These annual real rates are defined as the simple average of real rates from Belgium, Germany, France, Italy, the Netherlands, the United Kingdom, and the United States, where the country-specific annual real rates are determined using the following formula ${ }^{14}$

$$
\text { real rate }=\frac{\text { short-term nominal rate }- \text { inflation rate }}{1+\text { inflation rate }} .
$$

The resulting average annual real rate from 1961 to 2017 is shown in Figure 16 with a solid black line. Note that this series is characterized by significant and persistent fluctuations the past 55 years.

In the second step, the so-called "unrounded expected" real rate $R_{t}$ is calculated as the average of the annual real rate described above between 1961 and the current year:

$$
R_{1960+n}=\frac{1}{n} \sum_{i=1}^{n} r_{1960+i}
$$

where $n$ is the number of years between 1961 and the current year.

The applicable, or rounded, expected real rate, denoted $R_{t}^{r}$, is the unrounded expected real rate from equation (5) rounded to the nearest 5 basis points. When the unrounded rate is lower than the rounded rate of the previous year, i.e., $R_{t}<R_{t-1}^{r}$, the rate is rounded upwards. When the unrounded rate is higher than the rounded rate of the previous year, the rate is rounded downwards. Importantly, this expected real rate $R_{t}^{r}$ is common to all currencies and shown in Figure 16 with a solid red line. Note that the averaging built into the definition of $R_{t}^{r}$ implies that it will be an increasingly smooth function as time passes.

difficulties in selling those bonds back to the market in the future, which are unaccounted for in the DNS model.

${ }^{14}$ See EIOPA (2017c) for details. 


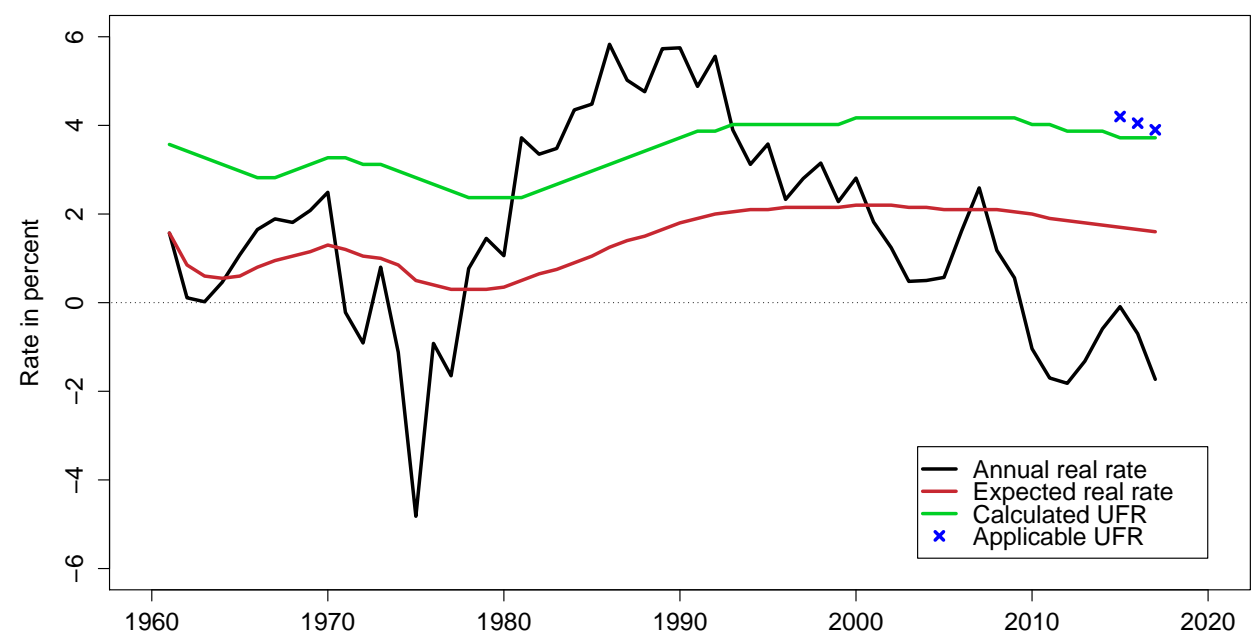

Figure 16: Key Components of the EIOPA UFR for the U.S.

Illustration of the annual real rate, the smoothed expected real rate, and the calculated UFR for the U.S. as detailed in EIOPA rules. These series are annual data starting in 1961. Also shown are the applicable U.S. $U F R^{A}$ values for 2015, 2016, and 2017.

In the third step, country-specific nominal rates are created by adding the expected inflation rate, which is tied to each central bank's inflation target and rounded to the following values: ${ }^{15}$

- $1 \%$ for inflation targets between 0 and $1 \%$;

- $2 \%$ for inflation targets between 1 and $3 \%$;

- $3 \%$ for inflation targets between 3 and $4 \%$;

- $4 \%$ for inflation targets of $4 \%$ or higher.

This determines the unadjusted $\mathrm{UFR}_{t}$ in year $t$ and represents a measure of the long-run steady state value of short-term rates in the given currency area. Note, as also emphasized below, this rate is calculated at the end of year $t$, announced in year $t+1$, and applicable in year $t+2$. Since the inflation target is specific to each central bank so is the UFR. For the U.S., these annual unadjusted $\mathrm{UFR}_{t}$ values are shown in Figure 16 with a solid green line.

The applicable or adjusted UFR, which is actually used to discount cash flows and denoted

\footnotetext{
${ }^{15}$ The inflation target is used where available and with a provision detailed in the methodology document otherwise.
} 


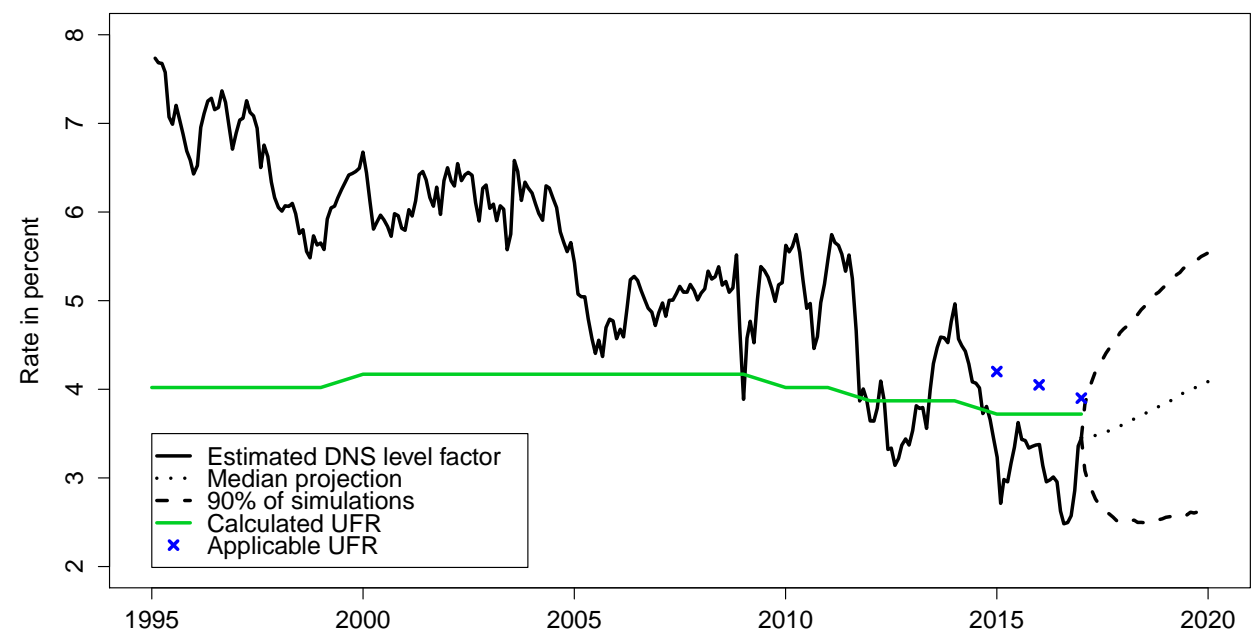

Figure 17: Comparison of EIOPA UFR for the U.S. and DNS Model Projections Illustration of the calculated UFR for the U.S. as detailed in EIOPA rules covering the period from 1995 to 2017. Also shown are the applicable U.S. $U F R^{A}$ for 2015, 2016, and 2017. For comparison we show the estimated level factor from the full sample U.S. DNS model described in Section 4.3 covering monthly data from January 31, 1995, to December 31, 2016, in addition to the median and $90 \%$ range of projections based on $N=10,000$ simulations through December 2019 from this model as detailed in Section 6.3.

$U F R_{t}^{A}$, is limited to a 15 basis-point annual change and is calculated as:

$$
U F R_{t}^{A}=\left\{\begin{array}{lll}
U F R_{t-1}^{A}+15 \mathrm{bps} & \text { if } & U F R_{t} \geq U F R_{t-1}^{A}+15 \mathrm{bps} \\
U F R_{t-1}^{A}-15 \mathrm{bps} & \text { if } & U F R_{t} \leq U F R_{t-1}^{A}-15 \mathrm{bps} \\
U F R_{t-1}^{A} & \text { otherwise } &
\end{array}\right.
$$

In particular, the calculated UFR for 2017 (based on data ending 2016 and applicable starting in January 2018) is 3.65\%, see EIOPA (2017b). As this represents a drop of more than 15 basis points from the $U F R^{A}$ of $4.2 \%$ applicable in 2017 that was enshrined in the "political settlement," which finalized Solvency II, see EIOPA (2017a), the $U F R^{A}$ applicable from 2018 is $4.05 \%$. The latest UFR calculation from EIOPA came out on 21 March 2018, see EIOPA (2018). The calculated UFR came out at $3.72 \%$, which represents another drop bigger than 15 basis points from the previous year, when it was $4.05 \%$. As a consequence, the $U F R^{A}$ applicable for 2019 will be $3.90 \%$. These applicable $U F R^{A}$ values are indicated with blue crosses in Figure 16.

In Figure 17, we compare the historically available calculated and applicable UFR values to the UFR implied by the U.S. DNS model as detailed in Section 5.1. We also show the projections for the following three years based on the associated model simulations described 
below in Section 6.3. We note that for the last ten years the UFR implied by the DNS model has fluctuated around the UFR ${ }_{t}$ values for the U.S. calculated under the EIOPA rules. Furthermore, the model projections suggest a convergence between the two measures in coming years. We take this as evidence of the robustness offered by the DNS model when it comes to extrapolating long-term yields.

\section{Risk Management Applications}

While point estimates of longer-term yields are needed for the projections and pricing exercises discussed above, projections of other elements characterizing the yield curve are needed for risk management purposes. In this section, we examine the properties of the proposed DNS model with respect to generating reasonable yield curve scenarios that extend to include longer-term yields. To do so, we estimate the DNS model on the Swiss and U.S. domestic datasets and simulate it to make a probability-based assessment of the interest rate outcomes as advocated by Christensen et al. (2015) as well as Christensen and Lopez (2015). ${ }^{16}$ Our analysis shows that the extrapolation errors of longer-term bond yields documented in the previous section are small relative to the yield changes required to observe stressful events in the tails of the model-implied bond yield distributions.

\subsection{Motivation}

Insurance companies, pension plans, and other financial institutions must deal with managing long-horizon portfolios, especially those with fixed-income bonds. Accurate models of yield curve forecasts are valuable, but accurate risk measurement is also important for managing these positions. As clearly noted by Engle et al. (2017), mean or median forecasts are not the only objects of interest; measures of extreme outcomes - such as value-at-risk (VaR) estimates that gauge the extent of possible tail outcomes - are also of importance to portfolio managers.

A particularly useful risk measurement outcome is determining the range of possible future yield curve values based on scenario analysis; that is, a modeling framework can be used to simulate many possible future yield curves and sketch out the range of possible outcomes. As highlighted in Christensen et al. (2012, 2015) as well as Christensen and Lopez (2015), such scenarios can be readily generated with an estimated model and used to generate probabilities of specific interest rate outcomes or related outcomes.

In this section, we generate yield curve scenarios and ground their reasonableness with comparisons with yield forecasts from surveys of professional forecasters. The main result is that the ranges of interest rate scenarios arising from a standard DNS model are sufficiently large to encompass these forecasts and are thus appropriate for scenario-based risk management purposes. Our analysis also suggests that the biases observed in Sections 3 and 4 for

\footnotetext{
${ }^{16}$ Results for Canada and France are reported in the online appendix.
} 


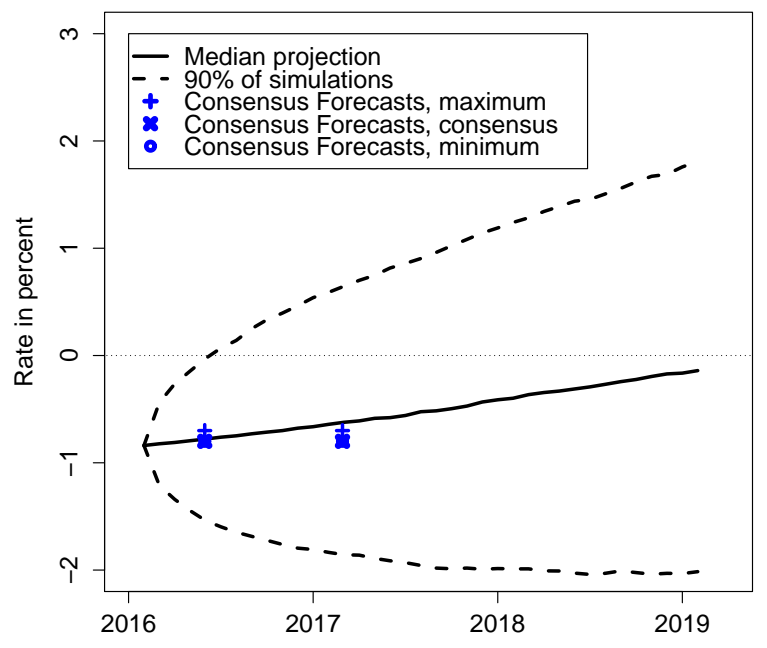

Figure 18: Three-Month Yield Projections from the Swiss DNS Model

Illustration of the median and [5\%,95\%] range of the three-month yield from the Swiss DNS model's simulated interest rate scenarios as of January 29, 2016. Also shown are the consensus forecasts as well as the maximum and minimum responses from the Consensus Forecasts survey performed on February 8,2016 .

the DNS model point estimates remain small in absolute terms and certainly quite small relative to the range of scenario outcomes. Accordingly, DNS modeling and extrapolation of longer-term yields, even those beyond the LLP, provides a richer set of useful diagnostics than simply using existing alternative methods.

\subsection{Interest Rate Scenarios Using the Swiss Data}

For this simulation exercise, we estimate the unrestricted DNS model on the full set of Swiss bonds; i.e., $\tau_{\max }=50$ years. The estimated parameters are reported in the last two columns of Table 2. The model fit is reported in the last two columns of Table 3 and shown in Figure 6. For all bonds combined, the RMSE is 9.37 basis points.

To measure the degree of yield variation that the DNS model dynamics can generate, we simulate 10,000 factor paths over a three-year horizon using as conditioning information the estimated parameters and factor values as of January 29, 2016. To keep things simple, we focus on the simulated paths for two yields: (1) the three-month yield, which is the model equivalent for the three-month Swiss franc LIBOR rate targeted by the Swiss National Bank, and (2) the projected ten-year Swiss Confederation bond yield, which is a widely used benchmark for many Swiss fixed-income securities.

For the short-rate projections, Figure 18 shows DNS projections for the three-month yield as of January 29, 2016. The figure shows the median, 5th, and 95th percentile values for 


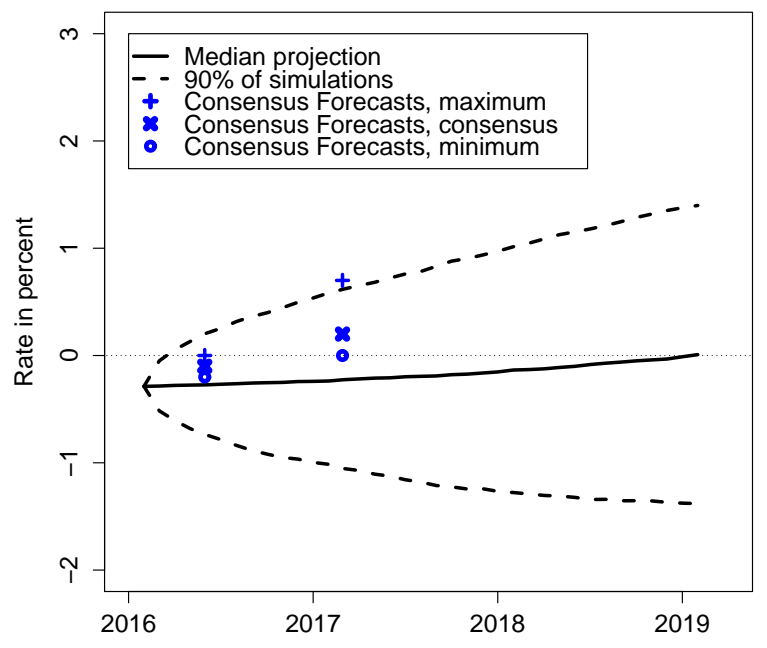

Figure 19: Ten-Year Yield Projections from the Swiss DNS Model

Illustration of the median and [5\%,95\%] range of the ten-year yield from the Swiss DNS model's simulated interest rate scenarios as of January 29, 2016. Also shown are the consensus forecasts as well as the maximum and minimum responses from the Consensus Forecasts survey performed on February 8, 2016.

the simulated three-month yield over a three-year forecast horizon. ${ }^{17}$ The median projections indicate a continued gradual normalization of monetary policy with the three-month yield reaching a level close to zero by the spring of 2019. The upper 95th percentile rises more rapidly, breaching zero in mid-2016 and approaching 2 percent at three years out. The lower 5 th percentile suggests a further drop in the three-month yield to about -2 percent. Note the increasing width of the $[5 \%, 95 \%]$ range. By the spring of 2019 , there is almost a 400 basis point difference between the top and the bottom of this range, which suggests a reasonably large degree of yield variation.

To provide perspective for these outcome projections, we compare them to the Consensus Forecasts survey conducted as of February 8, 2016. ${ }^{18}$ The survey contains responses to questions about the level of the three-month Swiss Confederation bond yields at month-end May 2016 and February 2017. The forecasts are very tightly clustered at just above -1 percent for both dates, and the model's median projection matches that pattern very closely.

Next, we focus on the simulated paths for the ten-year yield, which is a key benchmark for most financial institutions. ${ }^{19}$ Figure 19 illustrates its median and [5\%,95\%] range at each

\footnotetext{
${ }^{17}$ Note that the lines do not represent short rate paths from a single simulation run over the forecast horizon; instead, they delineate the distribution of all simulation outcomes at a given point in time. The same procedure is applied for the corresponding figures for all considered countries.

${ }^{18}$ Consensus Forecasts is produced by FocusEconomics using individual forecasts from expert panels for the major world economies.

${ }^{19}$ Results for the 50-year yields are qualitatively similar and therefore not shown for all considered countries.
} 


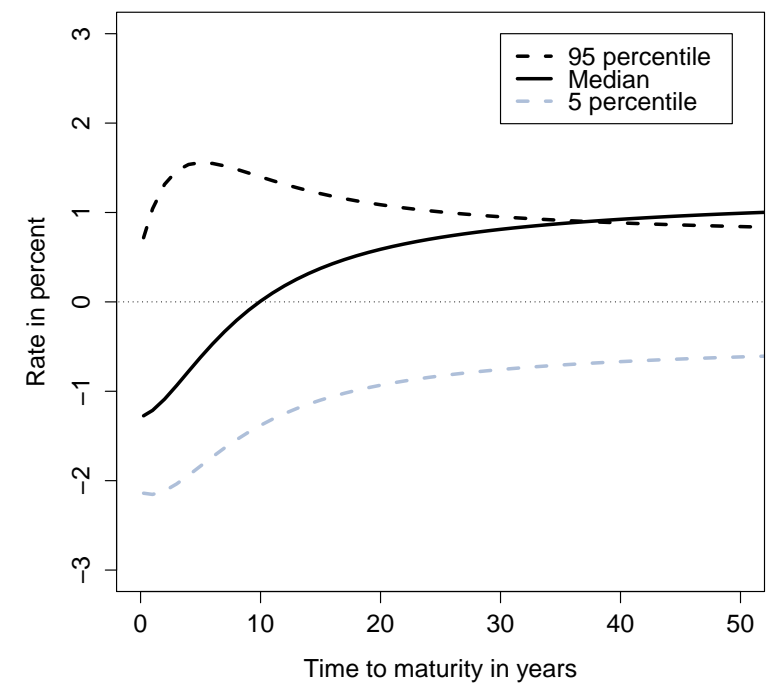

Figure 20: Simulated Swiss Yield Curves for January 2019

point in time from the simulated interest rate scenarios. The ten-year projections suggest that the likely range of outcomes at a three-year horizon is quite wide with a width of almost 300 basis points, which is only marginally smaller than the corresponding range of projected short rates. The median simulated ten-year yield indicates a continued gradual normalization, reaching zero at the end of the projection horizon. The upper 95th percentile rises more rapidly and goes above 1 percent at three years out, while the lower 5 th percentile represents a scenario with further declines in Swiss long-term interest rates.

For these projections, the Consensus Forecasts survey contains 11 responses to questions about the level of the ten-year Swiss Confederation bond yields at month-end May 2016 and February 2017. Figure 19 shows the consensus forecasts as well as the maximum and minimum response to those two questions. We note that the model-implied median projection is slightly lower than the path of the consensus survey forecast. Furthermore, the top of the projected range is below the maximum survey response. This alignment of yield projections is quite encouraging regarding the model's performance; i.e., the model's close match with the projections by professional forecasters when it is using a "yields-only" approach speaks well of its forecasting abilities. Correspondingly, the model's usefulness for risk management purposes seems quite high.

In Figure 20, we illustrate the entire yield curve corresponding to the three percentiles of the ten-year yield projections for the end of January 2019 (i.e., the rightmost projection point in the graph), as shown in Figure 19. Note the wide variation in the yield curve levels and shapes that the model suggests may occur with $90 \%$ probability over the next three years. This figure underscores that the DNS model is able to generate quite a wide range 


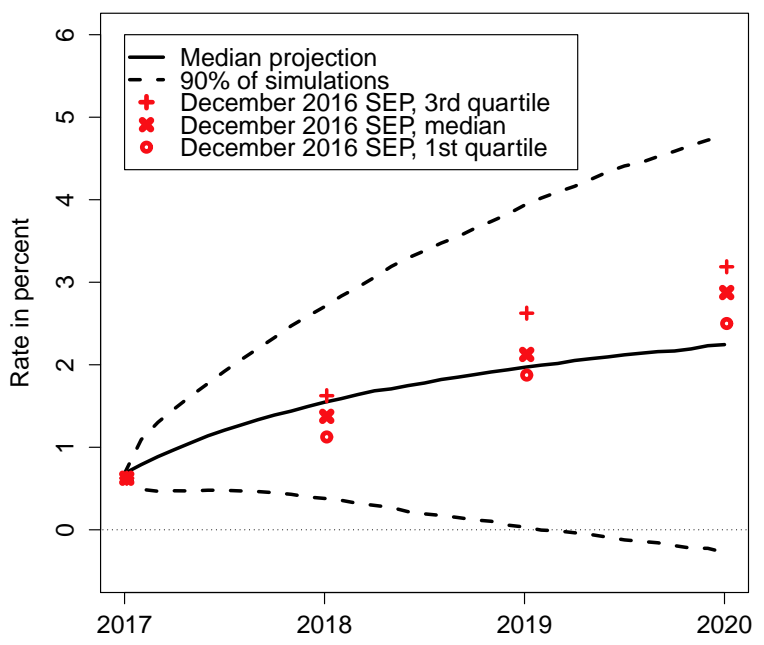

Figure 21: Short-Rate Projections from the U.S. DNS Model

Illustration of the median and [5\%,95\%] range of the fed funds rate from the U.S. Treasury DNS model's simulated interest rate scenarios as of December 30, 2016. Also shown are the median and the first and third quartile of the projections of future target levels for the overnight federal funds rate submitted by FOMC members at their December 2016 meeting as part of the Summary of Economic Projections (SEP).

of yield outcomes, even at the relatively short projection horizons often used for regulatory stress-testing.

\subsection{Interest Rate Scenarios Using the U.S. Treasury Data}

Recall that for the U.S. Treasury data, we use the GSW yields and not individual bond prices. The estimated parameters of the DNS model of U.S. Treasury yields are reported in the last two columns of Table 8. Its model fit is reported in the last two columns of Table 9. As already stated in Section 4.3, the model provides a very close fit to the entire cross section of available U.S. Treasury bond yields.

To give a sense of the amount of yield variation the DNS model dynamics can generate, we simulate 10,000 factor paths over a three-year horizon using as conditioning information the estimated parameters and factor values as of December 30, 2016. To keep things simple, we focus on the simulated paths for the short rate, which is the model equivalent for the overnight federal funds rate targeted by the Federal Open Market Committee (FOMC), and on the projected ten-year U.S. Treasury yields, which is a widely used benchmark for many U.S. fixed-income securities such as MBS.

To provide some insight into the variation in the resulting short-rate projections, Figure 21 compares forecasts of the short rate as of December 30, 2016, from the DNS model. The 


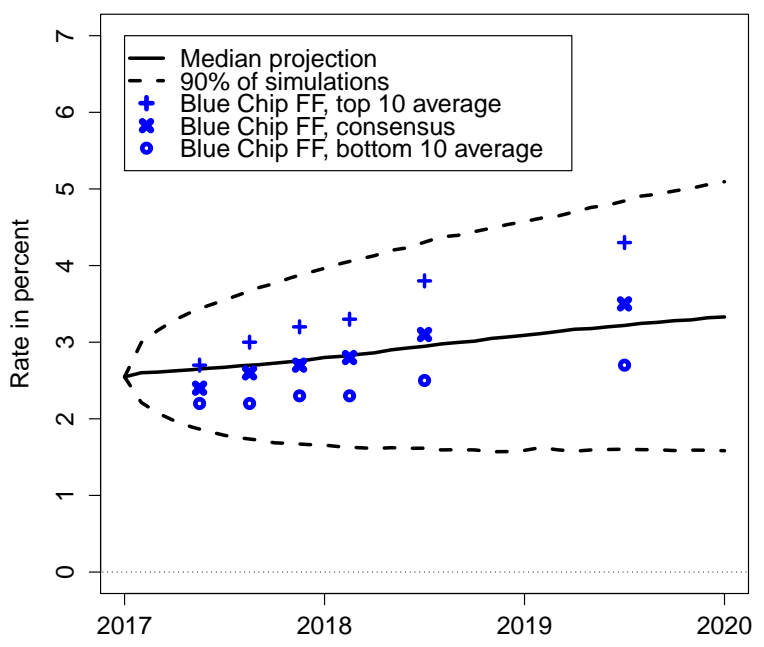

Figure 22: Ten-Year Treasury Yield Projections from the U.S. DNS Model

Illustration of the median and [5\%,95\%] range of the ten-year Treasury yield from the U.S. Treasury DNS model's simulated interest rate scenarios as of December 30, 2016. Also shown are the consensus forecasts as well as the top 10 and bottom 10 averages from the Blue Chip Financial Forecasts survey released on December 1, 2016.

figure shows the median, 5th, and 95th percentile values for the simulated short rate for a three-year forecast horizon. The median simulated short rate indicates a continued gradual normalization of monetary policy with the short rate reaching a level slightly above 2 percent by the end of 2019. The upper 95th percentile rises more rapidly and reaches 5 percent at three years out, while the lower 5th percentile represents a return to the zero lower bound in early 2019. Also shown are the lower and upper quartiles as well as the median of FOMC members' own expectations about appropriate future target rates for the overnight federal funds rate taken from their Summary of Economic Projections (SEP) released on December 14, 2016, following the December 2016 FOMC meeting. Given that the FOMC has the authority to set this target rate, these projections represent a useful benchmark for assessing the reasonableness of the model-implied projections. We note that the model-implied median projection is indeed very close to the median projection among the policy makers on the FOMC. Furthermore, note the width of the [5\%,95\%] range. By the end of 2019, there is a 500 basis point difference between the top and the bottom of this range.

Next, we focus on the simulated paths for the ten-year Treasury yield, which is a key benchmark for most financial institutions. Figure 22 illustrates the median and [5\%,95\%] range of the ten-year yield at each point in time from the simulated interest rate scenarios. The median simulated ten-year yield indicates a continued gradual normalization of the longterm interest rate environment. The upper 95th percentile rises more rapidly and reaches 5 


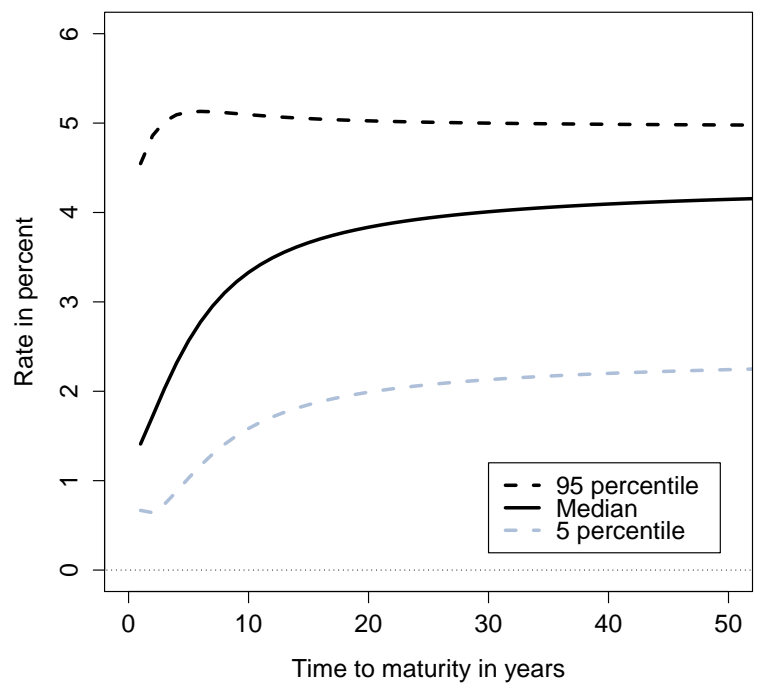

Figure 23: Simulated U.S. Yield Curves for December 2019

percent at three years out, while the lower 5th percentile represents a return to the very low interest rate environment that prevailed in the immediate years after the financial crisis.

Again, to provide perspective for the model projections, we compare them to survey forecasts, this time we use the long-term ten-year Treasury yield forecasts included in the Blue Chip Financial Forecasts survey released on December 1, 2016. In Figure 22, we show the Blue Chip consensus forecast as well as the averages of the bottom ten lowest and top ten highest forecasts. We note that the model-implied median projection is close to the path of the Blue Chip consensus forecast. Furthermore, the range of the projected ten-year yields fully encompass the considered range of forecasts in the Blue Chip survey. More importantly, the projections suggest the likely range of outcomes at a three-year horizon is quite wide with a width of about 400 basis points similar to what we saw for the projected short rates.

In Figure 23, we illustrate the entire yield curve corresponding to the three percentiles of the ten-year yield projections for the end of December 2019 shown in Figure 22. Note the wide variation in the shape of the yield curve that the model suggests may occur with $90 \%$ probability over the next three years. This figure underscores that the DNS model is able to generate quite a wide range of yield outcomes even at the relatively short projection horizons typically considered in regulatory stress tests of financial institutions.

Although the distribution of the generated scenarios could be evaluated in a more formal and comprehensive way as in Engle et al. (2017), the key takeaway for the analysis in this paper is that, thanks to the estimated high persistence of the level factor, the DNS model is able to generate quite a wide range of future interest rate environments despite its Gaussian dynamics. Thus, it is not clear that stochastic yield volatility needs to be incorporated into 
the model to achieve simulated outcomes that are adequately stressful with a reasonable likelihood as also noted by Christensen et al. (2015). Furthermore, this highlights that if simulations of the DNS model are used to generate yield curves for scenarios in stress tests of insurance companies, any potential bias in the extrapolated long bond yields is easily dwarfed by the variation in the overall yield level that the estimated model dynamics are able to generate over the projection horizons typically considered in stress test exercises. Thus, overall extrapolation errors of long bond yields would only represent a very modest distortion to the true yield curves in the relevant stressful interest rate scenarios.

\subsection{Summary}

The empirical results shown in this section highlight two insights. First, the yield curve scenarios that can be generated from DNS models are reasonable with respect to the yield curve projections from surveys of professional forecasters. Second, the extrapolation bias discussed earlier is sufficiently small that it does not interfere with the usefulness of the scenario projections. These two results - combined with the ease of DNS model estimation and applicability across countries and data sources (i.e., individual bond prices and composite yields at various maturities) - provide further evidence on the reasonableness of this approach for yield curve risk management, including at the longer-term maturities that extend beyond the maturities of traded securities.

\section{Conclusion}

In this paper, we analyze the ability of a standard term structure model, namely the DNS model of Diebold and Li (2006), to generate extrapolated long bond yields from the information in bond yields of shorter maturity.

First, we estimate the DNS model on data from the Swiss Confederation bond market where 50-year bonds have been trading for many years. The results indicate that, indeed, the DNS model performs well at extrapolating those 50-year yields using only the information in prices of bonds with maturities up to 30 years. Analysis of Canadian and French government bond prices confirms these empirical findings.

To assess the robustness of this conclusion, we simulate yield samples from a total of three different arbitrage-free affine term structure models and use those in estimations of the DNS model reported in the online appendix B. These exercises also indicate that the DNS model performs well at extrapolating long bond yields from the information in bond yields of shorter maturity despite being misspecified by construction. Therefore, this evidence combined leads us to recommend using DNS models as a tool to generate extrapolated long bond yields in general.

More importantly, in the context of stress tests where regulators tend to look for events 
in the tails of the relevant yield distributions, we note that the bias in the extrapolated long bond yields we detect in our analysis is easily dwarfed by the changes in yield levels required to observe such outcomes. Thus, for stress test purposes, our recommendation is particularly strong.

Finally, we emphasize the flexibility of the proposed approach based on the DNS model. First, its robust estimation based on the Kalman filter handles missing data in a straightforward way and easily accommodates nonlinear measurements such as bond prices instead of the more commonly used zero-coupon bond yields. Second, the baseline model can be extended to include additional asset classes such as TIPS (see Christensen et al. 2010) and corporate bonds (see Christensen and Lopez 2012). Third, if greater flexibility is needed to fit the observed data, the extension offered by Svensson (1995) can be explored in a similar manner. In short, it is an established, well-tested, rich, and flexible, yet very robust framework that we recommend to use as the foundation for generating reliable extrapolated long bond yields to be used in stress tests and other regulations for assessing and measuring the interest rate risk of insurance companies with long-lived liabilities. This modeling approach should readily accommodate the balance between stability of a key regulatory parameter (i.e., the UFR) and sensitivity to nearer-term economic and financial developments. Still, we acknowledge that there are other methods for modeling, projecting, and assessing interest rate risk in the long run; see Engle et al. (2017) as well as the Smith-Wilson modeling approach adapted by EIOPA and reviewed by Gourieroux and Monfort (2015). We leave a comparison with these alternatives for future research. 


\section{References}

Andreasen, Martin M., Jens H. E. Christensen, and Glenn D. Rudebusch, 2019, "Term Structure Analysis with Big Data: One-Step Estimation Using Bond Prices," forthcoming Journal of Econometrics.

Christensen, Jens H. E., Francis X. Diebold, and Glenn D. Rudebusch, 2011, "The Affine Arbitrage-Free Class of Nelson-Siegel Term Structure Models," Journal of Econometrics, Vol. 164, No. 1, 4-20.

Christensen, Jens H. E. and Signe Krogstrup, 2019, "Transmission of Quantitative Easing: The Role of Central Bank Reserves," Economic Journal, Vol. 129, 249-272.

Christensen, Jens H. E. and Jose A. Lopez, 2012, "Common Risk Factors in the US Treasury and Corporate Bond Markets: An Arbitrage-Free Dynamic Nelson-Siegel Modeling Approach," Manuscript, Federal Reserve Bank of San Francisco.

Christensen, Jens H. E. and Jose A. Lopez, 2015, "Assessing Supervisory Scenarios for Interest Rate Risk," Federal Reserve Bank of San Francisco, Economic Letter 2015-29.

Christensen, Jens H. E., Jose A. Lopez, and Glenn D. Rudebusch, 2010, "Inflation Expectations and Risk Premiums in an Arbitrage-Free Model of Nominal and Real Bond Yields," Journal of Money, Credit and Banking, Supplement to Vol. 42, No. 6, 143-178.

Christensen, Jens H. E., Jose A. Lopez, and Glenn D. Rudebusch, 2012, "Extracting Deflation Probability Forecasts from Treasury Yields," International Journal of Central Banking, Vol. 8, No. 4, 21-60.

Christensen, Jens H. E., Jose A. Lopez, and Glenn D. Rudebusch, 2015, "A ProbabilityBased Stress Test of Federal Reserve Assets and Income," Journal of Monetary Economics, Vol. 73, 26-43.

Christensen, Jens H. E. and Glenn D. Rudebusch, 2012, "The Response of Interest Rates to U.S. and U.K. Quantitative Easing," Economic Journal, Vol. 122, F385-F414.

Christensen, Jens H. E. and Glenn D. Rudebusch, 2015, "Estimating Shadow-Rate Term Structure Models with Near-Zero Yields," Journal of Financial Econometrics, Vol. 13, No. 2, 226-259.

Christensen, Jens H. E. and Glenn D. Rudebusch, 2016, "Modeling Yields at the Zero Lower Bound: Are Shadow Rates the Solution?," in Eric Hillebrand, Siem Jan Koopman (ed.) Dynamic Factor Models (Advances in Econometrics, Volume 35) Emerald Group Publishing Limited, 75-125. 
Christensen, Jens H. E. and Glenn D. Rudebusch, 2019, “A New Normal for Interest Rates? Evidence from Inflation-Indexed Debt," forthcoming Review of Economics and Statistics.

Christensen, Jens H. E., Glenn D. Rudebusch, and Patrick Shultz, 2018, "A New Normal for Interest Rates? Evidence from Canadian Government Bond Prices," Manuscript, Federal Reserve Bank of San Francisco.

Diebold, Francis X. and Canlin Li, 2006, "Forecasting the Term Structure of Government Bond Yields," Journal of Econometrics, Vol. 130, 337-364.

Diebold, Francis X., Glenn D. Rudebusch and S. Boragan Aruoba, 2006, "The Macroeconomy and the Yield Curve: A Dynamic Latent Factor Approach," Journal of Econometrics, Vol. 131, 309-338.

EIOPA, 2017a, "Frequently Asked Questions \& Answers. THE ULTIMATE FORWARD RATE," available at the link:

https://eiopa.europa.eu/Publications/Reports/Frequently\%20Asked\%20Questions\%20and\%20Answers\%20UFR.pdf

EIOPA, 2017b, "Risk-free interest rate term structures. Calculation of the UFR for 2018," available at the link: https://eiopa.europa.eu/Publications/Reports/Calculation\%20of\%20the\%20UFR\%20for\%202018.pdf

EIOPA, 2017c, "Risk-free interest rate term structures. Specification of the methodology to derive the UFR," available at the link:

https://eiopa.europa.eu/Publications/Reports/Specification\%20of\%20the\%20methodology\%20to\%20derive\%20the\%20UFR.pdf

EIOPA, 2018a, "Risk-free interest rate term structures. Report on the Calculation of the UFR for 2019," available at the link: https://eiopa.europa.eu/Publications/Reports/Calculation\%20of\%20the\%20UFR\%20for\%202019.pdf

EIOPA, 2018b, "Technical documentation of the methodology to derive EIOPA's risk-free interest rate term structures," available at the link: https://eiopa.europa.eu/Publications/Standards/Technical\%20Documentation\%20\%2831\%20Jan\%202018\%29.pdf

Engle, Robert, Guillaume Roussellet, and Emil Siriwardane, 2017, "Scenario Generation for Long Run Interest Rate Risk Assessment," Journal of Econometrics, Vol. 201, No. 2, 333-347.

Garbade, Kenneth D., 2017a, "Beyond 30: Long-Term Treasury Bond Issuance from 1953 to 1957," Liberty Street Economics, February 6.

Garbade, Kenneth D., 2017b, "Beyond 30: Long-Term Treasury Bond Issuance from 1957 to 1965," Liberty Street Economics, February 8. 
Gourieroux, C. and A. Monfort, 2015, "A Note on the Smith-Wilson Family," Manuscript, CREST.

Gürkaynak, Refet S., Brian Sack, and Jonathan H. Wright, 2007, "The U.S. Treasury Yield Curve: 1961 to the Present," Journal of Monetary Economics, Vol. 54, No. 8, 22912304 .

Kim, Don H. and Kenneth J. Singleton, 2012, "Term Structure Models and the Zero Bound: An Empirical Investigation of Japanese Yields," Journal of Econometrics, Vol. 170, No. $1,32-49$.

Laubach, T. and J.C. Williams, 2016. "Measuring the Natural Rate of Interest Redux," Business Economics, April, 57-67.

Nelson, Charles R. and Andrew F. Siegel, 1987, "Parsimonious Modeling of Yield Curves," Journal of Business, Vol. 60, No. 4, 473-489.

Quaedvlieg, Rogier and Peter Schotman, 2016, "Score-Driven Nelson-Siegel: Hedging LongTerm Liabilities," Manuscript, Erasmus School of Economics.

Svensson, Lars E. O., 1995, "Estimating Forward Interest Rates with the Extended NelsonSiegel Method," Quarterly Review, No. 3, Sveriges Riksbank, 13-26.

Zigraiova, Daniel and Petr Jakubik, 2017, "Updating the Ultimate Forward Rate over Time: A Possible Approach," Working paper 2017-3, Czech National Bank. 


\title{
Online Appendix
}

\section{Extrapolating Long-Maturity Bond Yields for Financial Risk Measurement}

\author{
Jens H. E. Christensen \\ Federal Reserve Bank of San Francisco \\ jens.christensen@sf.frb.org \\ and \\ Jose A. Lopez \\ Federal Reserve Bank of San Francisco \\ jose.a.lopez@sf.frb.org \\ and \\ Paul L. Mussche \\ Federal Reserve Bank of San Francisco \\ paul.mussche@sf.frb.org
}

The views in this paper are solely the responsibility of the authors and should not be interpreted as reflecting the views of the Federal Reserve Bank of San Francisco or the Board of Governors of the Federal Reserve System.

This version: March 22, 2019. 


\section{Contents}

A The Kalman Filter Estimation of DNS Models 2

B Simulation Study on Swiss Data 5

B.1 The General Approach . . . . . . . . . . . . . . . . . . . . 5

B.2 The Three AF Models Used for Simulations . . . . . . . . . . . . . . . 6

B.2.1 The CV Model . . . . . . . . . . . . . . . 6

B.2.2 The SV Model . . . . . . . . . . . . . . . . . . . 7

B.2.3 The GCV Model . . . . . . . . . . . . . . . . . . . . . 9

B.3 Model Estimation Results . . . . . . . . . . . . . . . . . . . . . 11

B.4 Details of the Simulations . . . . . . . . . . . . . . . . . 14

B.5 CV Model Results . . . . . . . . . . . . . . . . . . 16

B.6 SV Model Results . . . . . . . . . . . . . . . . . . . . 17

B.7 GCV Model Results . . . . . . . . . . . . . . . . . . . . . . 18

B.8 Summary . . . . . . . . . . . . . . . . . . . . . . . . . . . . 19

C Additional Results for Canada $\quad 19$

C.1 Interest Rate Scenarios Using the Canadian Data . . . . . . . . . . . . . . . 19

D Additional Results for France $\quad 25$

D.1 Interest Rate Scenarios Using the French Data . . . . . . . . . . . . 25 


\section{A The Kalman Filter Estimation of DNS Models}

In this appendix, we detail how we estimate the DNS models using the Kalman filter.

To begin, denote the three state variables by the vector $X_{t}=\left(L_{t}, S_{t}, C_{t}\right)$, then the state equation can be written on the following affine form

$$
X_{t}=\Phi_{0}(\psi)+\Phi_{1}(\psi) X_{t-1}+\eta_{t}, \quad \eta_{t} \sim N(0, Q),
$$

where

$$
\begin{aligned}
& \Phi_{0}(\psi)=(I-A) \mu, \\
& \Phi_{1}(\psi)=A .
\end{aligned}
$$

Here, $\Phi_{0}(\psi)$ and $\Phi_{1}(\psi)$ are fixed throughout the estimation. Note that the time between observations are implicit in the equations above.

The observation equation is also affine

$$
y=\Lambda X_{t}+\varepsilon .
$$

Now the actual steps in the Kalman filter algorithm can be outlined. Define the total information available at time $t$ by

$$
Y_{t}=\left(y_{1}, y_{2}, \ldots, y_{t}\right)
$$

and denote the best guess of the state variables in the last period by $\widehat{X}_{t-1}$ and the best guess

of their mean square error matrix by $\widehat{\Sigma}_{t-1}$. In the prediction step of the Kalman filter the best guess of the current value of $X_{t}$ given the information set from the last period, $Y_{t-1}$, is calculated as

$$
\widehat{X}_{t \mid t-1}=E^{\mathbb{P}}\left[X_{t} \mid Y_{t-1}\right]=\Phi_{0}+\Phi_{1} \widehat{X}_{t-1}
$$

The corresponding prediction for the mean square error matrix is

$$
\widehat{\Sigma}_{t \mid t-1}=\Phi_{1}(\psi) \widehat{\Sigma}_{t-1} \Phi_{1}(\psi)^{\prime}+Q
$$

In the update step the predicted value is improved upon by using the additional information 
contained in $Y_{t}$. The minimum error solution to this problem is given by the equations

$$
\begin{aligned}
& \widehat{X}_{t}=E^{\mathbb{P}}\left[X_{t} \mid Y_{t}\right]=\widehat{X}_{t \mid t-1}+\widehat{\Sigma}_{t \mid t-1} \Lambda^{\prime} F_{t}^{-1} v_{t}, \\
& \widehat{\Sigma}_{t}=\widehat{\Sigma}_{t \mid t-1}-\widehat{\Sigma}_{t \mid t-1} \Lambda^{\prime} F_{t}^{-1} \Lambda \widehat{\Sigma}_{t \mid t-1},
\end{aligned}
$$

where $^{1}$

$$
\begin{aligned}
v_{t} & =y_{t}-E^{\mathbb{P}}\left[y_{t} \mid Y_{t-1}\right]=y_{t}-\Lambda \widehat{X}_{t \mid t-1}, \\
F_{t} & =\operatorname{cov}\left(v_{t}\right)=\Lambda \widehat{\Sigma}_{t \mid t-1} \Lambda^{\prime}+H_{t} .
\end{aligned}
$$

For a given set of parameters $\psi$ the logarithm of the likelihood function is calculated as ${ }^{2}$

$$
\log L\left(y_{1}, \ldots, y_{T} ; \psi\right)=\sum_{t=1}^{T}\left(-\frac{m_{t}}{2} \log (2 \pi)-\frac{1}{2} \log \left|F_{t}\right|-\frac{1}{2} v_{t}^{\prime} F_{t}^{\prime} v_{t}\right)
$$

The optimal choice of the parameters $\psi$ and the corresponding most likely path of $X_{t}$ are found by maximizing the value of the log-likelihood function with respect to $\psi$ using the Nelder-Mead simplex algorithm. Upon convergence, the standard errors are obtained from the estimated covariance matrix,

$$
\widehat{\Omega}(\widehat{\psi})=\frac{1}{T}\left[\frac{1}{T} \sum_{t=1}^{T} \frac{\partial \log l_{t}(\widehat{\psi})}{\partial \psi} \frac{\partial \log l_{t}(\widehat{\psi})^{\prime}}{\partial \psi}\right]^{-1}
$$

where $\widehat{\psi}$ denotes the estimated model parameters.

Finally, there is the important decision to make as to where to start the Kalman filter algorithm, i.e., choosing the initial value of the state variables. Diebold et al. (2006) start the algorithm at the unconditional mean and variance of the state variables, which is equivalent to assuming that the state variables are all stationary processes. The VAR(1) process for the $X_{t}$-process above is stationary if the length of all the eigenvalues of the matrix $A$ is smaller than 1, which is assumed in the following and imposed in the Kalman filter optimization.

According to Harvey (1989) p. 120-121 the unconditional mean is given by

$$
E^{\mathbb{P}}[X]=\mu,
$$

\footnotetext{
${ }^{1}$ The dimension of $H_{t}$ is determined by the number of bond yields observed at time $t$.

${ }^{2} m_{t}$ is the number of observed yields at time $t$.
} 
while the unconditional variance $V$ is given implicitly as the solution to the following equation

$$
V=A V A^{\prime}+Q
$$

Once this system is solved, the Kalman filter algorithm can be started with the following initial values

$$
\widehat{X}_{0}=\mu \quad \text { and } \quad \widehat{\Sigma}_{0}=V .
$$

In the model estimations using bond prices, the extended Kalman filter is needed because the measurement equations are no longer affine functions of the state variables, see equation (3) in the paper. Instead, the measurement equation takes the general form

$$
\frac{\bar{P}_{t}^{i}\left(\tau^{i}, C^{i}\right)}{D_{t}\left(\tau^{i}, C^{i}\right)}=z\left(X_{t} ; \tau^{i}, C^{i}, \psi\right)+\varepsilon_{t}^{i}
$$

where $\bar{P}_{t}^{i}\left(\tau^{i}, C^{i}\right)$ is the observed clean price (i.e., without accrued interest) of bond $i$ at time $t$, $D_{t}\left(\tau^{i}, C^{i}\right)$ is its duration calculated before model estimation based on the Macaulay definition, while $z\left(X_{t} ; \tau^{i}, C^{i}, \psi\right)$ is given by the nonlinear function

$$
z\left(X_{t} ; \tau^{i}, C^{i}, \psi\right)=\frac{\widehat{P}_{t}\left(\tau^{i}, C^{i}\right)}{D_{t}\left(\tau^{i}, C^{i}\right)}
$$

where $\widehat{P}_{t}\left(\tau^{i}, C^{i}\right)$ is the model-implied clean bond price described in equation (2) in the paper.

In the extended Kalman filter, equation (1) is linearized using a first-order Taylor expansion around the best guess of $X_{t}$ in the prediction step of the Kalman filter algorithm. Thus, in the notation introduced above, this best guess is denoted $X_{t \mid t-1}$ and the approximation is given by

$$
z\left(X_{t} ; \tau^{i}, C^{i}, \psi\right) \approx z\left(X_{t \mid t-1} ; \tau^{i}, C^{i}, \psi\right)+\left.\frac{\partial z\left(X_{t} ; \tau^{i}, C^{i}, \psi\right)}{\partial X_{t}}\right|_{X_{t}=X_{t \mid t-1}}\left(X_{t}-X_{t \mid t-1}\right)
$$

Thus, by defining

$$
\begin{aligned}
& A_{t}(\psi) \equiv z\left(X_{t \mid t-1} ; \tau^{i}, C^{i}, \psi\right)-\left.\frac{\partial z\left(X_{t} ; \tau^{i}, C^{i}, \psi\right)}{\partial X_{t}}\right|_{X_{t}=X_{t \mid t-1}} X_{t \mid t-1}, \\
& \left.B_{t}(\psi) \equiv \frac{\partial z\left(X_{t} ; \tau^{i}, C^{i}, \psi\right)}{\partial X_{t}}\right|_{X_{t}=X_{t \mid t-1}},
\end{aligned}
$$


the measurement equation can be given on an affine form as

$$
\frac{\bar{P}_{t}^{i}\left(\tau^{i}, C^{i}\right)}{D_{t}\left(\tau^{i}, C^{i}\right)}=A_{t}(\psi)+B_{t}(\psi) X_{t}+\varepsilon_{t}^{i}
$$

and the steps in the algorithm proceed as previously described by letting $\Lambda_{t}=B_{t}(\psi)$.

\section{B Simulation Study on Swiss Data}

Although we consider data from four countries in the main text, the analysis still leaves unaddressed questions about how reliable extrapolated yields derived from the DNS model are provided the observed data is generated by an arbitrage-free dynamic term structure model. From Krippner (2015) it follows that the proposed approach may work very well in theory, but in this appendix, we assess that conjecture more formally by simulating yield samples from three different arbitrage-free models estimated on the Swiss data analyzed in Section 3 of the paper. Since all three models are affine, one caveat of the simulation exercise to note up front is that the effects of nonlinearities in the yield curve are left unexplored. Although we note that, given the good fit of the DNS model, which is also affine, there is presumably little room for any significant nonlinear effects. However, if deemed necessary, we stress that it is straightforward to include nonlinear models in the analysis such as the one described in Feldhütter et al. (2016), but we leave that for future research.

The analysis in this appendix proceeds as follows. First, we explain the steps and logic behind the simulation study we undertake. Second, we introduce the three dynamic term structure models used to generate simulated bond yields. Third, we describe the technical details of the model simulations before we proceed to an analysis of the results. Overall, the key purpose is to demonstrate how reliable the DNS model is at extrapolating long-term yields, even when it is misspecified by construction.

\section{B.1 The General Approach}

First, we simulate arbitrage-free dynamic term structure models and generate zero-coupon yields with maturities $\tau$ up to 50 years, $\tau \in\{0.25,0.5,1,2,3,5,7,10,15,20,30,50\}$. We stress that the parameters used in the simulations are obtained from model estimations based on the Swiss bond price data with maturities up to 50 years.

Second, we use the simulated samples as input into DNS model estimations with varying 
maximum yield maturity: 10 years, 20 years, and 30 years, respectively. For comparison we also make corresponding estimations of the true model that generated the data.

Finally, we calculate the extrapolation errors from the DNS model estimations and compare them to the extrapolation errors made by the true model. This should provide a fair assessment of the ability of the DNS model to extrapolate long bond yields as a misspecified representation of the actual yield dynamics.

\section{B.2 The Three AF Models Used for Simulations}

In this section, we briefly describe the three arbitrage-free affine dynamic term structure models we use in the simulation study.

\section{B.2.1 The CV Model}

We first focus on the arbitrage-free equivalent of the DNS model known as the arbitrage-free class of Nelson-Siegel (AFNS) models introduced in Christensen et al. (2011). Since this model is characterized by constant volatility, we refer to it as the CV model. In this class of models, the state vector is denoted by $X_{t}=\left(L_{t}, S_{t}, C_{t}\right)$, where $L_{t}$ is a level factor, $S_{t}$ is a slope factor, and $C_{t}$ is a curvature factor. The instantaneous risk-free rate is defined as

$$
r_{t}=L_{t}+S_{t}
$$

To preserve the Nelson and Siegel (1987) factor loading structure in the yield function, the risk-neutral (or $\mathbb{Q}$-) dynamics of the state variables are given by the stochastic differential equations ${ }^{3}$

$$
\left(\begin{array}{l}
d L_{t} \\
d S_{t} \\
d C_{t}
\end{array}\right)=\left(\begin{array}{ccc}
0 & 0 & 0 \\
0 & -\lambda & \lambda \\
0 & 0 & -\lambda
\end{array}\right)\left(\begin{array}{c}
L_{t} \\
S_{t} \\
C_{t}
\end{array}\right) d t+\Sigma\left(\begin{array}{c}
d W_{t}^{L, \mathbb{Q}} \\
d W_{t}^{S, \mathbb{Q}} \\
d W_{t}^{C, \mathbb{Q}}
\end{array}\right)
$$

where $\Sigma$ is the constant covariance (or volatility) matrix. ${ }^{4}$ Based on this specification of the $\mathbb{Q}$-dynamics, zero-coupon bond yields preserve the Nelson-Siegel factor loading structure as

$$
y_{t}(\tau)=L_{t}+\left(\frac{1-e^{-\lambda \tau}}{\lambda \tau}\right) S_{t}+\left(\frac{1-e^{-\lambda \tau}}{\lambda \tau}-e^{-\lambda \tau}\right) C_{t}-\frac{A(\tau)}{\tau},
$$

\footnotetext{
${ }^{3}$ As discussed in Christensen et al. (2011), with a unit root in the level factor, the model is not arbitragefree with an unbounded horizon; therefore, as is often done in theoretical discussions, we impose an arbitrary maximum horizon.

${ }^{4}$ As per Christensen et al. (2011), $\Sigma$ is a diagonal matrix, and $\theta^{\mathbb{Q}}$ is set to zero without loss of generality.
} 
where the yield-adjustment term is given by

$$
\begin{aligned}
\frac{A(\tau)}{\tau}= & \frac{\sigma_{11}^{2}}{6} \tau^{2}+\sigma_{22}^{2}\left[\frac{1}{2 \lambda^{2}}-\frac{1}{\lambda^{3}} \frac{1-e^{-\lambda \tau}}{\tau}+\frac{1}{4 \lambda^{3}} \frac{1-e^{-2 \lambda \tau}}{\tau}\right] \\
& +\sigma_{33}^{2}\left[\frac{1}{2 \lambda^{2}}+\frac{1}{\lambda^{2}} e^{-\lambda \tau}-\frac{1}{4 \lambda} \tau e^{-2 \lambda \tau}-\frac{3}{4 \lambda^{2}} e^{-2 \lambda \tau}+\frac{5}{8 \lambda^{3}} \frac{1-e^{-2 \lambda \tau}}{\tau}-\frac{2}{\lambda^{3}} \frac{1-e^{-\lambda \tau}}{\tau}\right] .
\end{aligned}
$$

So far, the description of the CV model has relied solely on the dynamics of the state variables under the $\mathbb{Q}$-measure used for pricing. However, to complete the description of the model and to implement it empirically, we will need to specify the risk premiums that connect the factor dynamics under the $\mathbb{Q}$-measure to the dynamics under the real-world (or historical) $\mathbb{P}$-measure. It is important to note that there are no restrictions on the dynamic drift components under the empirical $\mathbb{P}$-measure beyond the requirement of constant volatility. To facilitate empirical implementation, we use the essentially affine risk premium specification introduced in Duffee (2002). In the Gaussian framework, this specification implies that the risk premiums $\Gamma_{t}$ depend on the state variables; that is,

$$
\Gamma_{t}=\gamma^{0}+\gamma^{1} X_{t}
$$

where $\gamma^{0} \in \mathbf{R}^{3}$ and $\gamma^{1} \in \mathbf{R}^{3 \times 3}$ contain unrestricted parameters. Thus, the resulting unrestricted three-factor $\mathrm{CV}$ model has $\mathbb{P}$-dynamics given by

$$
\left(\begin{array}{l}
d L_{t} \\
d S_{t} \\
d C_{t}
\end{array}\right)=\left(\begin{array}{ccc}
\kappa_{11}^{\mathbb{P}} & \kappa_{12}^{\mathbb{P}} & \kappa_{13}^{\mathbb{P}} \\
\kappa_{21}^{\mathbb{P}} & \kappa_{22}^{\mathbb{P}} & \kappa_{23}^{\mathbb{P}} \\
\kappa_{31}^{\mathbb{P}} & \kappa_{32}^{\mathbb{P}} & \kappa_{33}^{\mathbb{P}}
\end{array}\right)\left(\left(\begin{array}{c}
\theta_{1}^{\mathbb{P}} \\
\theta_{2}^{\mathbb{P}} \\
\theta_{3}^{\mathbb{P}}
\end{array}\right)-\left(\begin{array}{c}
L_{t} \\
S_{t} \\
C_{t}
\end{array}\right)\right) d t+\Sigma\left(\begin{array}{c}
d W_{t}^{L, \mathbb{P}} \\
d W_{t}^{S, \mathbb{P}} \\
d W_{t}^{C, \mathbb{P}}
\end{array}\right)
$$

This is the transition equation in the Kalman filter estimation.

\section{B.2.2 The SV Model}

Financial time series, such as interest rates and bond yields, have been shown to have timevarying volatility, which is a feature not often incorporated into arbitrage-free term structure models; see Andersen and Benzoni (2010) for further discussion. To address this concern, Christensen et al. (2014) develop a general class of AFNS models that incorporate spanned stochastic volatility. To distinguish between the various types of models, we use the notation outlined in Dai and Singleton (2000) for classifying affine term structure models, such that 
the CV model is within the $A_{0}(3)$ class of models that do not have volatility dynamics. As detailed in Christensen et al. (2014), there are several possible volatility specifications within their three-factor framework.

For our analysis, we choose an $A_{1}(3)$ volatility specification that incorporates stochastic volatility based on the level factor. For this stochastic volatility (SV) model, the state vector and instantaneous risk-free rates are the same as before. To preserve the Nelson-Siegel factor loading structure and impose our volatility specification, the $\mathbb{Q}$-dynamics of the state variables are given by

$$
\begin{aligned}
\left(\begin{array}{l}
d L_{t} \\
d S_{t} \\
d C_{t}
\end{array}\right) & =\left(\begin{array}{ccc}
\kappa_{L}^{\mathbb{Q}} & 0 & 0 \\
0 & \lambda & -\lambda \\
0 & 0 & \lambda
\end{array}\right)\left[\left(\begin{array}{c}
\theta_{L}^{\mathbb{Q}} \\
0 \\
0
\end{array}\right)-\left(\begin{array}{c}
L_{t} \\
S_{t} \\
C_{t}
\end{array}\right)\right] d t \\
& +\left(\begin{array}{ccc}
\sigma_{11} & 0 & 0 \\
\sigma_{21} & \sigma_{22} & 0 \\
\sigma_{31} & \sigma_{32} & \sigma_{33}
\end{array}\right)\left(\begin{array}{ccc}
\sqrt{L_{t}} & 0 & 0 \\
0 & \sqrt{1+\beta_{21} L_{t}} & 0 \\
0 & 0 & \sqrt{1+\beta_{31} L_{t}}
\end{array}\right)\left(\begin{array}{c}
d W_{t}^{L, \mathbb{Q}} \\
d W_{t}^{S, \mathbb{Q}} \\
d W_{t}^{C, \mathbb{Q}}
\end{array}\right) .
\end{aligned}
$$

The representation of the zero-coupon bond yield function becomes ${ }^{5}$

$$
y_{t}(\tau)=g\left(\kappa_{L}^{\mathbb{Q}}\right) L_{t}+\left(\frac{1-e^{-\lambda \tau}}{\lambda \tau}\right) S_{t}+\left(\frac{1-e^{-\lambda \tau}}{\lambda \tau}-e^{-\lambda \tau}\right) C_{t}-\frac{A\left(\tau ; \kappa_{L}^{\mathbb{Q}}\right)}{\tau},
$$

where $g^{N}\left(\kappa_{L}^{\mathbb{Q}}\right)$ is a modified loading on the level factor. Note that the slope and the curvature factor preserve their Nelson-Siegel factor loadings exactly, although the structure of the yieldadjustment term $A\left(\tau ; \kappa_{L}^{\mathbb{Q}}\right) / \tau$ is different than before. ${ }^{6}$

To link the risk-neutral and real-world dynamics of the state variables, we here use the extended affine risk premium specification introduced by Cheridito et al. (2007), as per Christensen et al. (2014). The maximally flexible affine specification of the $\mathbb{P}$-dynamics is thus

\footnotetext{
${ }^{5}$ In our implementation, we fix $\kappa_{L}^{\mathbb{Q}}=10^{-6}$ to get a close approximation to the uniform level factor loading in the $\mathrm{CV}$ model.

${ }^{6}$ Note that both $g\left(\kappa_{L}^{\mathbb{Q}}\right)$ and $A\left(\tau ; \kappa_{L}^{\mathbb{Q}}\right)$ must be calculated numerically by solving a system of ODEs, see Christensen et al. (2014) for details.
} 


$$
\begin{aligned}
\left(\begin{array}{l}
d L_{t} \\
d S_{t} \\
d C_{t}
\end{array}\right) & =\left(\begin{array}{ccc}
\kappa_{11}^{\mathbb{P}} & 0 & 0 \\
\kappa_{21}^{\mathbb{P}} & \kappa_{22}^{\mathbb{P}} & \kappa_{23}^{\mathbb{P}} \\
\kappa_{31}^{\mathbb{P}} & \kappa_{32}^{\mathbb{P}} & \kappa_{33}^{\mathbb{P}}
\end{array}\right)\left[\left(\begin{array}{c}
\theta_{1}^{\mathbb{P}} \\
\theta_{2}^{\mathbb{P}} \\
\theta_{3}^{\mathbb{P}}
\end{array}\right)-\left(\begin{array}{c}
L_{t} \\
S_{t} \\
C_{t}
\end{array}\right)\right] d t \\
& +\left(\begin{array}{ccc}
\sigma_{11} & 0 & 0 \\
\sigma_{21} & \sigma_{22} & 0 \\
\sigma_{31} & \sigma_{32} & \sigma_{33}
\end{array}\right)\left(\begin{array}{ccc}
\sqrt{L_{t}} & 0 & 0 \\
0 & \sqrt{1+\beta_{21} L_{t}} & 0 \\
0 & 0 & \sqrt{1+\beta_{31} L_{t}}
\end{array}\right)\left(\begin{array}{c}
d W_{t}^{L, \mathbb{P}} \\
d W_{t}^{S, \mathbb{P}} \\
d W_{t}^{C, \mathbb{P}}
\end{array}\right) .
\end{aligned}
$$

To keep the model arbitrage-free, the level factor must be prevented from hitting the lower zero-boundary. This positivity requirement is ensured by imposing the Feller conditions under both probability measures, which in this case are two; that is,

$$
\kappa_{11}^{\mathbb{P}} \theta_{1}^{\mathbb{P}}>\frac{1}{2} \sigma_{11}^{2} \quad \text { and } \quad 10^{-6} \cdot \theta_{L}^{\mathbb{Q}}>\frac{1}{2} \sigma_{11}^{2}
$$

\section{B.2.3 The GCV Model}

The main in-sample problem with the regular Nelson-Siegel model is that, for reasonable choices of $\lambda$ (which are empirically in the vicinity of 0.5 for U.S. Treasury yield data), the factor loading for the slope and the curvature factor decay rapidly to zero as a function of maturity. Thus, the model mostly relies on the level factor for its fit to yields with ten years or more to maturity. Empirically, this limitation shows up as a lack of fit of the long-term yields, as described in Christensen et al. (2011).

For this reason we also consider a generalized AFNS model introduced in Christensen et al. (2009) that includes an additional slope and curvature factor. Since this model is also characterized by constant volatility and represents a generalization of the CV model, we refer to it as the GCV model.

In this class of models, the vector of state variables is $X_{t}=\left(L_{t}, S_{t}^{1}, S_{t}^{2}, C_{t}^{1}, C_{t}^{2}\right)$, while the instantaneous risk-free rate is defined by

$$
r_{t}=L_{t}+S_{t}^{1}+S_{t}^{2}
$$

To generate the appropriate Nelson and Siegel (1987) factor loading structure in the yield function, the risk-neutral (or $\mathbb{Q}$-) dynamics of the state variables are given by the stochastic differential equations 


$$
\left(\begin{array}{l}
d L_{t} \\
d S_{t}^{1} \\
d S_{t}^{2} \\
d C_{t}^{1} \\
d C_{t}^{2}
\end{array}\right)=\left(\begin{array}{ccccc}
0 & 0 & 0 & 0 & 0 \\
0 & -\lambda_{1} & 0 & \lambda_{1} & 0 \\
0 & 0 & -\lambda_{2} & 0 & \lambda_{2} \\
0 & 0 & 0 & -\lambda_{1} & 0 \\
0 & 0 & 0 & 0 & -\lambda_{2}
\end{array}\right)\left(\begin{array}{c}
L_{t} \\
S_{t}^{1} \\
S_{t}^{2} \\
C_{t}^{1} \\
C_{t}^{2}
\end{array}\right) d t+\Sigma\left(\begin{array}{c}
d W_{t}^{L, \mathbb{Q}} \\
d W_{t}^{S^{1}, \mathbb{Q}} \\
d W_{t}^{S^{2}, \mathbb{Q}} \\
d W_{t}^{C^{1}, \mathbb{Q}} \\
d W_{t}^{C^{2}, \mathbb{Q}}
\end{array}\right), \quad \lambda_{1}>\lambda_{2}>0
$$

where we continue to assume that $\Sigma$ is a diagonal matrix.

In this model, zero-coupon bond yields are given by

$$
y_{t}(\tau)=L_{t}+\frac{1-e^{-\lambda_{1} \tau}}{\lambda_{1} \tau} S_{t}^{1}+\frac{1-e^{-\lambda_{2} \tau}}{\lambda_{2} \tau} S_{t}^{2}+\left[\frac{1-e^{-\lambda_{1} \tau}}{\lambda_{1} \tau}-e^{-\lambda_{1} \tau}\right] C_{t}^{1}+\left[\frac{1-e^{-\lambda_{2} \tau}}{\lambda_{2} \tau}-e^{-\lambda_{2} \tau}\right] C_{t}^{2}-\frac{A^{G}(\tau)}{\tau}
$$

where $\frac{A^{G}(\tau)}{\tau}$ represents a generalized yield-adjustment, see Christensen et al. (2009) for details.

Similar to the CV model, the description of the GCV model is completed by specifying the risk premiums that connect the factor dynamics under the $\mathbb{Q}$-measure to the dynamics under the real-world (or historical) $\mathbb{P}$-measure. To facilitate empirical implementation, we continue to use the essentially affine risk premium specification introduced in Duffee (2002). In the Gaussian framework, this specification implies that the risk premiums $\Gamma_{t}$ take the following form

$$
\Gamma_{t}=\gamma^{0}+\gamma^{1} X_{t}
$$

where $\gamma^{0} \in \mathbf{R}^{5}$ and $\gamma^{1} \in \mathbf{R}^{5 \times 5}$ contain unrestricted parameters.

Thus, the resulting unrestricted five-factor GCV model has $\mathbb{P}$-dynamics given by

$$
\left(\begin{array}{c}
d L_{t} \\
d S_{t}^{1} \\
d S_{t}^{2} \\
d C_{t}^{1} \\
d C_{t}^{2}
\end{array}\right)=\left(\begin{array}{ccccc}
\kappa_{11}^{\mathbb{P}} & \kappa_{12}^{\mathbb{P}} & \kappa_{13}^{\mathbb{P}} & \kappa_{14}^{\mathbb{P}} & \kappa_{15}^{\mathbb{P}} \\
\kappa_{21}^{\mathbb{P}} & \kappa_{22}^{\mathbb{P}} & \kappa_{23}^{\mathbb{P}} & \kappa_{24}^{\mathbb{P}} & \kappa_{25}^{\mathbb{P}} \\
\kappa_{31}^{\mathbb{P}} & \kappa_{32}^{\mathbb{P}} & \kappa_{33}^{\mathbb{P}} & \kappa_{34}^{\mathbb{P}} & \kappa_{35}^{\mathbb{P}} \\
\kappa_{41}^{\mathbb{P}} & \kappa_{42}^{\mathbb{P}} & \kappa_{43}^{\mathbb{P}} & \kappa_{44}^{\mathbb{P}} & \kappa_{45}^{\mathbb{P}} \\
\kappa_{51}^{\mathbb{P}} & \kappa_{52}^{\mathbb{P}} & \kappa_{53}^{\mathbb{P}} & \kappa_{54}^{\mathbb{P}} & \kappa_{55}^{\mathbb{P}}
\end{array}\right)\left(\left(\begin{array}{c}
\theta_{1}^{\mathbb{P}} \\
\theta_{2}^{\mathbb{P}} \\
\theta_{3}^{\mathbb{P}} \\
\theta_{4}^{\mathbb{P}} \\
\theta_{5}^{\mathbb{P}}
\end{array}\right)-\left(\begin{array}{c}
L_{t} \\
S_{t}^{1} \\
S_{t}^{2} \\
C_{t}^{1} \\
C_{t}^{2}
\end{array}\right)\right) d t+\Sigma\left(\begin{array}{c}
d W_{t}^{L, \mathbb{P}} \\
d W_{t}^{S^{1}, \mathbb{P}} \\
d W_{t}^{S^{2}, \mathbb{P}} \\
d W_{t}^{C^{1}, \mathbb{P}} \\
d W_{t}^{C^{2}, \mathbb{P}}
\end{array}\right) .
$$

This is the transition equation in the Kalman filter estimation. 


\begin{tabular}{|c|ccc||c||c|c|}
\hline$K^{\mathbb{P}}$ & $K_{, 1}^{\mathbb{P}}$ & $K_{\cdot, 2}^{\mathbb{P}}$ & $K_{\cdot, 3}^{\mathbb{P}}$ & $\theta^{\mathbb{P}}$ & & $\Sigma$ \\
\hline$K_{1, \cdot}^{\mathbb{P}}$ & 0.0141 & 0 & 0 & 0.0368 & $\sigma_{11}$ & 0.0030 \\
& $(0.0379)$ & & & $(0.0068)$ & & $(0.0001)$ \\
$K_{2, \cdot}^{\mathbb{P}}$ & 0 & 0.1923 & 0 & -0.0267 & $\sigma_{22}$ & 0.0078 \\
& & $(0.1326)$ & & $(0.0072)$ & & $(0.0002)$ \\
$K_{3, \cdot}^{\mathbb{P}}$ & 0 & 0 & 0.8351 & -0.0116 & $\sigma_{33}$ & 0.0174 \\
& & & $(0.2720)$ & $(0.0046)$ & & $(0.0010)$ \\
\hline
\end{tabular}

\section{Table 1: Estimated Parameters for the CV Model}

The table shows the estimated parameters of the $K^{\mathbb{P}}$ matrix, $\theta^{\mathbb{P}}$ vector, and diagonal $\Sigma$ matrix for the CV model with independent factors. The estimated value of $\lambda$ is 0.2383 (0.0029). The maximum log likelihood value is $30,733.89$. The numbers in parentheses are the estimated parameter standard deviations.

\begin{tabular}{|c|ccc||c||c|c||c|c|}
\hline$K^{\mathbb{P}}$ & $K_{\cdot, 1}^{\mathbb{P}}$ & $K_{\cdot, 2}^{\mathbb{P}}$ & $K_{\cdot, 3}^{\mathbb{P}}$ & $\theta^{\mathbb{P}}$ & & $\Sigma$ & & $\beta$ \\
\hline$K_{1, \cdot}^{\mathbb{P}}$ & 0.0092 & 0 & 0 & 0.0332 & $\sigma_{11}$ & 0.0162 & $\beta_{11}$ & n.a. \\
& $(0.0253)$ & & & $(0.0063)$ & & $(0.0003)$ & & \\
$K_{2, \cdot}^{\mathbb{P}}$ & 0 & 0.2284 & 0 & -0.0220 & $\sigma_{22}$ & 0.0002 & $\beta_{21}$ & 36,616 \\
& & $(0.1591)$ & & $(0.0061)$ & & $(0.0000)$ & & $(167.5)$ \\
$K_{3, \cdot}^{\mathbb{P}}$ & 0 & 0 & 0.7802 & -0.0112 & $\sigma_{33}$ & 0.0161 & $\beta_{31}$ & 0.0005 \\
& & & $(0.2665)$ & $(0.0043)$ & & $(0.0024)$ & & $(6.7833)$ \\
\hline
\end{tabular}

Table 2: Estimated Parameters for the SV Model

The table shows the estimated parameters of the $K^{\mathbb{P}}$ matrix, $\theta^{\mathbb{P}}$ vector, diagonal $\Sigma$ matrix, and $\beta$ vector for the SV model with independent factors. The estimated value of $\lambda$ is 0.2771 (0.0033), while the estimated value of $\theta_{L}^{\mathbb{Q}}$ is 131.4 (170.7). The maximum log likelihood value is 30,629.52. The numbers in parentheses are the estimated parameter standard deviations.

\section{B.3 Model Estimation Results}

In this section, we describe the results obtained when estimating the CV, SV, and GCV models on the sample of Swiss Confederation bond prices. We stress up front that, to minimize the computing time in the simulation study, we only consider the most parsimonious specification of each model where all factors are independent, which is without loss of generality for our conclusions.

Table 1 reports the estimated parameters for the CV model, which is the arbitrage-free equivalent of the DNS model. We note the usual pattern that the level factor is the most persistent and least volatile factor, the curvature factor is the most volatile and least persistent factor, and the slope factor has dynamic properties in between those two extremes. Finally, the estimated value of $\lambda$ is close to 0.25 , which is notably lower than the value estimated with the DNS model.

Table 2 reports the estimated parameters for the SV model. Mostly, the estimated dy- 


\begin{tabular}{|c|c|c|c|c|c|c|c|c|}
\hline$K^{\mathbb{P}}$ & $K_{\cdot, 1}^{\mathbb{P}}$ & $K_{\cdot, 2}^{\mathbb{P}}$ & $K_{\cdot, 3}^{\mathbb{P}}$ & $K_{\cdot, 4}^{\mathbb{P}}$ & $K_{\cdot, 5}^{\mathbb{P}}$ & $\theta^{\mathbb{P}}$ & & $\Sigma$ \\
\hline$K_{1,}^{\mathbb{P}}$ & $\begin{array}{c}0.0083 \\
(0.0265)\end{array}$ & 0 & 0 & 0 & 0 & $\begin{array}{c}0.0406 \\
(0.0087)\end{array}$ & $\sigma_{11}$ & $\begin{array}{c}0.0026 \\
(0.0001)\end{array}$ \\
\hline$K_{2,}^{\mathbb{P}}$ & 0 & $\begin{array}{c}1.7693 \\
(0.4114)\end{array}$ & 0 & 0 & 0 & $\begin{array}{c}0.0037 \\
(0.0281)\end{array}$ & $\sigma_{22}$ & $\begin{array}{c}0.0198 \\
(0.0012)\end{array}$ \\
\hline$K_{3,}^{\mathbb{P}}$ & 0 & 0 & $\begin{array}{c}0.1665 \\
(0.1320)\end{array}$ & 0 & 0 & $\begin{array}{l}-0.0286 \\
(0.0104)\end{array}$ & $\sigma_{33}$ & $\begin{array}{c}0.0089 \\
(0.0005)\end{array}$ \\
\hline$K_{4}^{\mathbb{P}}$ & 0 & 0 & 0 & $\begin{array}{c}0.3890 \\
(0.2340)\end{array}$ & 0 & $\begin{array}{l}-0.0106 \\
(0.0174)\end{array}$ & $\sigma_{44}$ & $\begin{array}{c}0.0253 \\
(0.0022)\end{array}$ \\
\hline$K_{5,}^{\mathbb{P}}$ & 0 & 0 & 0 & 0 & $\begin{array}{c}0.2927 \\
(0.1499)\end{array}$ & $\begin{array}{l}-0.0074 \\
(0.0113)\end{array}$ & $\sigma_{55}$ & $\begin{array}{c}0.0159 \\
(0.0010)\end{array}$ \\
\hline
\end{tabular}

Table 3: Estimated Parameters for the GCV Model

The table shows the estimated parameters of the $K^{\mathbb{P}}$ matrix, $\theta^{\mathbb{P}}$ vector, and diagonal $\Sigma$ matrix for the GCV model with independent factors. The estimated value of $\lambda_{1}$ is 2.5507 (0.0629), while the estimated value of $\lambda_{2}$ is 0.1648 (0.0037). The maximum log likelihood value is $31,710.10$. The numbers in parentheses are the estimated parameter standard deviations.

namic parameters are very similar to those obtained for the CV model. Specifically, it remains the case that the level factor is the most persistent and least volatile factor, the curvature factor is the most volatile and least persistent factor, and the slope factor has dynamic properties in between those two extremes. Finally, the estimated value of $\lambda$ is also close to the value obtained for the $\mathrm{CV}$ model.

Table 3 reports the estimated parameters for the GCV model. We note that the estimated values of $\lambda_{1}$ and $\lambda_{2}$ are above and below, respectively, the values obtained for the CV and SV models. This means that the first pair of slope and curvature factors is used to fit the short end of the yield curve, while the second pair is used to improve the fit of medium- and longer-term yields. Furthermore, the level factor remains very persistent and with dynamic properties similar to those observed in the other two models.

Table 4 contains the summary statistics for the fitted errors of the three arbitrage-free models used in the simulation exercise with a comparison to the fit obtained with the DNS model. First, we note that the DNS, CV, and SV models all give a very similar fit to the data across the entire cross section of bond prices. Most surprisingly, this also holds for the two 50-year bonds. Thus, the arbitrage-free models are not able to exploit the additional yield-adjustment term in their discount functions to improve the fit of long-term bond prices. This is also consistent with the findings reported by Christensen et al. (2011).

Second, it is not a surprise that the GCV model with its five state variables is able to 


\begin{tabular}{|c|c|c|c|c|c|c|c|c|}
\hline \multirow{2}{*}{ Confederation bond } & \multicolumn{2}{|c|}{ DNS model } & \multicolumn{2}{|c|}{ CV model } & \multicolumn{2}{|c|}{ SV model } & \multicolumn{2}{|c|}{ GCV model } \\
\hline & Mean & RMSE & Mean & RMSE & Mean & RMSE & Mean & RMSE \\
\hline (1) $6.75 \% ~ 1 / 22 / 1999$ & 10.53 & 16.52 & 11.02 & 17.03 & 10.85 & 16.86 & 99.68 & 15.06 \\
\hline (2) $6.25 \% 3 / 15 / 1999$ & 5.14 & 17.08 & 5.55 & 17.21 & 5.42 & 17.26 & 5.26 & 15.99 \\
\hline (3) $6.25 \% 7 / 15 / 1999$ & -6.52 & 18.25 & -6.42 & 18.30 & -6.49 & 18.30 & -3.59 & 13.41 \\
\hline (4) $6.25 \% 7 / 15 / 2000$ & 3.12 & 17.36 & 2.14 & 18.06 & 2.39 & 17.84 & 4.29 & 15.30 \\
\hline (5) $6.5 \% 2 / 5 / 2002$ & 4.35 & 9.07 & 3.97 & 8.77 & 4.03 & 8.83 & 2.26 & 6.06 \\
\hline (6) $6.5 \% 4 / 10 / 2004$ & 1.51 & 8.19 & 1.61 & 8.29 & 1.69 & 8.24 & 0.38 & 6.59 \\
\hline (7) $6.75 \% 6 / 11 / 2003$ & 2.49 & 8.73 & 2.19 & 9.37 & 2.37 & 9.16 & 1.59 & 6.91 \\
\hline (8) $7 \% 7 / 9 / 2001$ & 2.26 & 7.70 & 1.64 & 7.73 & 1.75 & 7.63 & 0.42 & 6.36 \\
\hline (9) $7 \% 9 / 10 / 2003$ & 3.19 & 12.26 & 3.06 & 12.25 & 3.21 & 12.26 & 2.76 & 9.98 \\
\hline (10) $6.25 \% \quad 11 / 5 / 2004$ & 4.48 & 9.35 & 4.72 & 9.61 & 4.75 & 9.59 & 3.91 & 7.80 \\
\hline (11) $6.25 \% 1 / 7 / 2003$ & 2.32 & 8.33 & 1.58 & 8.79 & 1.87 & 8.62 & 1.35 & 5.98 \\
\hline (12) $5.25 \% 2 / 11 / 1998$ & -4.12 & 12.51 & -3.27 & 14.08 & -3.52 & 13.64 & -4.32 & 11.00 \\
\hline (13) $5 \% 3 / 11 / 2000$ & -4.40 & 11.28 & -5.09 & 11.46 & -4.91 & 11.36 & -2.61 & 10.36 \\
\hline (14) $4.5 \% 4 / 8 / 2006$ & -1.96 & 6.36 & -1.40 & 6.74 & -1.47 & 6.57 & -1.79 & 4.71 \\
\hline (15) $4.5 \% 6 / 10 / 2000$ & -5.14 & 10.85 & -6.06 & 10.78 & -5.84 & 10.75 & -4.18 & 10.74 \\
\hline (16) $4.5 \%$ 7/8/2002 & -2.67 & 8.85 & -3.41 & 9.41 & -3.19 & 9.25 & -3.59 & 5.67 \\
\hline (17) $4.5 \% \quad 10 / 7 / 2004$ & -1.89 & 5.92 & -1.56 & 5.95 & -1.53 & 5.92 & -2.77 & 4.98 \\
\hline (18) $4.25 \% 1 / 6 / 2014^{*}$ & 1.39 & 6.26 & 2.41 & 8.00 & 1.82 & 7.65 & 3.57 & 5.75 \\
\hline (19) $4 \% 3 / 10 / 1999$ & -9.48 & 13.91 & -8.88 & 13.51 & -9.12 & 13.68 & -9.35 & 14.49 \\
\hline (20) $5.5 \% \quad 10 / 7 / 2001$ & 0.94 & 10.62 & 0.35 & 10.24 & 0.40 & 10.29 & -1.28 & 7.79 \\
\hline (21) $5 \%$ 11/10/1996 & -1.53 & 12.60 & -4.05 & 17.45 & -2.75 & 15.69 & 0.91 & 6.04 \\
\hline (22) $5.5 \% 1 / 6 / 2005$ & -0.41 & 6.17 & -0.02 & 6.20 & -0.01 & 6.20 & -1.08 & 4.57 \\
\hline (23) $4.25 \%$ 1/8/2008 & -2.73 & 8.12 & -1.59 & 7.38 & -1.82 & 7.66 & -1.49 & 4.98 \\
\hline (24) $4.5 \% 6 / 10 / 2007$ & -2.68 & 7.47 & -1.86 & 7.11 & -1.95 & 7.13 & -1.24 & 4.44 \\
\hline (25) $4.25 \% 6 / 5 / 2017^{*}$ & 1.45 & 5.91 & -0.42 & 5.85 & -0.24 & 6.12 & 2.54 & 4.81 \\
\hline (26) $3.5 \% 8 / 7 / 2010$ & -1.05 & 9.57 & 0.74 & 9.69 & 0.07 & 9.74 & 2.05 & 8.44 \\
\hline (27) $3.25 \% 2 / 11 / 2009$ & 2.33 & 13.32 & 3.97 & 13.35 & 3.54 & 13.40 & 1.86 & 5.65 \\
\hline (28) $4 \% 2 / 11 / 2023^{\dagger}$ & 5.62 & 9.44 & 3.34 & 8.24 & 3.83 & 8.74 & 1.77 & 5.99 \\
\hline (29) $4 \% 4 / 8 / 2028^{+}$ & 7.00 & 10.44 & 4.43 & 9.30 & 5.23 & 9.80 & 1.63 & 7.72 \\
\hline (30) $4 \% 1 / 6 / 2049^{\times}$ & 1.14 & 12.47 & 3.96 & 11.46 & 4.05 & 13.33 & 2.29 & 7.50 \\
\hline (31) $2.75 \% 6 / 10 / 2012$ & -3.64 & 8.00 & -2.18 & 7.95 & -2.84 & 8.28 & -1.08 & 4.57 \\
\hline (32) $4 \%$ 2/11/2013 & -0.06 & 6.05 & 1.36 & 6.94 & 0.73 & 7.03 & 2.19 & 4.00 \\
\hline (33) $4 \%$ 6/10/2011 & 0.33 & 7.08 & 2.17 & 7.36 & 1.54 & 7.38 & 2.01 & 6.15 \\
\hline (34) $3.75 \% 6 / 10 / 2015$ & -1.28 & 4.68 & -1.10 & 5.25 & -1.44 & 5.36 & 0.92 & 3.67 \\
\hline (35) $3 \% 1 / 8 / 2018$ & -0.39 & 5.15 & -2.00 & 5.79 & -1.71 & 6.02 & 0.13 & 4.17 \\
\hline (36) $2.5 \% 3 / 12 / 2016$ & -2.98 & 5.32 & -3.37 & 6.00 & -3.36 & 6.17 & -1.32 & 4.39 \\
\hline (37) $3.5 \% 4 / 8 / 2033^{+}$ & -1.40 & 6.48 & -2.71 & 8.06 & -2.32 & 7.92 & -3.26 & 7.89 \\
\hline (38) $3 \%$ 5/12/2019 & -0.48 & 5.59 & -2.19 & 6.29 & -1.74 & 6.46 & -1.42 & 4.48 \\
\hline (39) $1.75 \%$ 11/5/2009 & -13.75 & 26.99 & -12.62 & 27.31 & -13.00 & 27.36 & -12.50 & 18.13 \\
\hline (40) $2.25 \% 7 / 6 / 2020$ & 0.48 & 4.88 & -1.04 & 5.00 & -0.50 & 5.19 & -1.70 & 4.23 \\
\hline (41) $2 \% 10 / 12 / 2016$ & -3.82 & 6.50 & -5.00 & 7.44 & -4.71 & 7.36 & -2.32 & 5.86 \\
\hline (42) $2 \% 11 / 9 / 2014$ & -8.88 & 13.19 & -7.84 & 13.83 & -8.26 & 13.71 & -6.96 & 11.47 \\
\hline (43) $2.5 \% 3 / 8 / 2036^{+}$ & -5.97 & 8.74 & -6.28 & 8.88 & -6.22 & 9.33 & -5.50 & 7.89 \\
\hline (44) $3.25 \% 6 / 27 / 2027^{*}$ & 4.61 & 7.38 & 4.19 & 7.61 & 4.52 & 7.90 & 1.06 & 4.63 \\
\hline (45) $2 \% 4 / 28 / 2021$ & 1.20 & 4.42 & 0.70 & 4.11 & 1.06 & 4.47 & -1.70 & 3.63 \\
\hline (46) $2 \% 5 / 25 / 2022$ & 1.68 & 4.27 & 2.10 & 4.09 & 2.28 & 4.46 & -0.94 & 3.23 \\
\hline (47) $2.25 \% 6 / 22 / 2031^{*}$ & 0.70 & 3.45 & 0.34 & 4.07 & 0.76 & 4.35 & -1.49 & 3.62 \\
\hline (48) $1.5 \% 4 / 30 / 2042^{+}$ & -9.33 & 11.03 & -10.92 & 11.57 & -10.65 & 11.81 & -7.32 & 7.82 \\
\hline (49) $1.25 \% 6 / 11 / 2024$ & 0.84 & 4.69 & 2.39 & 4.51 & 2.41 & 4.94 & -1.69 & 3.27 \\
\hline (50) $1.25 \% 6 / 27 / 2037^{\dagger}$ & -6.11 & 7.15 & -7.85 & 8.24 & -7.04 & 7.79 & -6.28 & 6.75 \\
\hline (51) $1.5 \% 7 / 24 / 2025$ & 1.38 & 4.76 & 3.38 & 5.14 & 3.26 & 5.33 & -0.41 & 2.40 \\
\hline (52) $1.25 \% 5 / 28 / 2026$ & -1.20 & 2.34 & 1.13 & 2.39 & 0.93 & 2.28 & -0.55 & 2.23 \\
\hline (53) $2 \% 6 / 25 / 2064^{\times}$ & -5.88 & 9.20 & -0.06 & 8.22 & -7.38 & 12.05 & -0.82 & 6.43 \\
\hline (54) $0.5 \% 5 / 27 / 2030$ & -4.67 & 4.95 & -3.34 & 3.44 & -2.56 & 2.90 & -3.96 & 4.13 \\
\hline All yields & -0.10 & $\overline{9.42}$ & -0.16 & 9.59 & -0.15 & 9.71 & -0.15 & 7.39 \\
\hline
\end{tabular}

Table 4: Summary Statistics of Fitted Errors of Swiss Confederation Bond Yields The table shows the mean fitted errors and the root mean-squared fitted errors (RMSE) of the yield to maturity for individual Swiss Confederation bonds according to the independent-factors DNS, CV, SV, and GCV models estimated with all available Swiss Confederation bonds. Asterisk * indicates twenty-year bonds, dagger $\dagger$ indicates twenty-five-year bonds, plus + indicates thirty-year bonds, and cross $\times$ indicates fifty-year bonds whose fit is highlighted in bold. The data are monthly and cover the period from January 29, 1993, to January 29, 2016. All numbers are measured in basis points. 
outperform the other models at fitting the entire cross section of bond prices, which is similar to the results reported in Christensen et al. (2009).

\section{B.4 Details of the Simulations}

To keep things simple, the simulation exercise is centered around the independent-factors specification of each model described above. Thus, it is these specifications and their estimated parameters reported in Tables 1 to 3 that we use in the simulations.

Based on those estimated parameters for each model, we first simulate $N=100$ sample paths for the state variables observed at a monthly frequency for ten years. In a second step, these simulated factor paths are converted into standard zero-coupon yields for the $\mathrm{CV}, \mathrm{SV}$, and GCV models using equations (4), (7), and (11), respectively. The simulated yield samples contain zero-coupon yields with a total of 12 maturities: $0.25,0.5,1,2,3,5,7,10,15,20,30$, and 50 years. A Gaussian i.i.d. measurement error with zero mean and a standard deviation $\sigma_{\varepsilon}$ of 10 basis points is added to each simulated yield. Note that, to make the exercises across models as comparable as possible, it is the same set of simulated Gaussian i.i.d. measurement errors that is used in each type of simulated yield data independent of the simulated model. Since the measurement equation for zero-coupon yields within the three model classes and the DNS model is affine, it follows that the model estimations based on the simulated yield samples can be performed with the standard Kalman filter unlike the estimations based on the actual bond price data that require nonlinear filtering.

We now turn to the details of the simulation of the factor paths. The continuous-time $\mathbb{P}$-dynamics in affine models are, in general, given by

$$
d X_{t}=K^{\mathbb{P}}\left(\theta^{\mathbb{P}}-X_{t}\right) d t+\Sigma D\left(X_{t}\right) d W_{t}^{\mathbb{P}}
$$

For both restricted square-root processes and unconstrained processes the continuous-time dynamics can be approximated using the Euler approximation. ${ }^{7}$ To exemplify, for a restricted square-root process,

$$
d X_{t}^{i}=\kappa_{i i}^{\mathbb{P}}\left(\theta_{i}^{\mathbb{P}}-X_{t}^{i}\right) d t+\kappa_{i j}^{\mathbb{P}}\left(\theta_{j}^{\mathbb{P}}-X_{t}^{j}\right) d t+\sigma_{i i} \sqrt{X_{t}^{i}} d W_{t}^{\mathbb{P}, i}
$$

\footnotetext{
${ }^{7}$ Thompson (2008) is an example.
} 
the algorithm is

$$
X_{t}^{i}=X_{t-1}^{i}+\kappa_{i i}^{\mathbb{P}}\left(\theta_{i}^{\mathbb{P}}-X_{t-1}^{i}\right) \Delta t+\kappa_{i j}^{\mathbb{P}}\left(\theta_{j}^{\mathbb{P}}-X_{t-1}^{j}\right) \Delta t+\sigma_{i i} \sqrt{X_{t-1}^{i}} \sqrt{\Delta t} z_{t}^{i}, \quad z_{t}^{i} \sim N(0,1) .
$$

We fix $\Delta t$ at a uniform value of 0.0001 to get a close approximation to the true factor dynamics. In the SV model, Feller conditions are imposed in the model estimations, which ensures that its parameter set used in the simulations satisfy all nonnegativity requirements, so the "true" underlying continuous-time process never becomes negative $\mathbb{P}$-a.s. However, for the discretely observed process above, there is always a positive, but usually very small probability that the approximation will become negative. Whenever this happens, we truncate the simulated square-root processes at zero similar to what we do in the estimation of the SV model.

As for the starting point of the simulation algorithm, $X_{0}$, we could draw that from the unconditional joint distribution of the state variables in the CV and GCV models thanks to their Gaussian dynamics. However, for the SV model and for affine models in general, we do not know the unconditional distribution of the state variables. Therefore, to keep our approach amenable to affine and non-affine models in general, we take the estimated value of the state variables at the end of the Swiss bond price sample and use them as the starting point to simulate the models according to the algorithm above for 100 years. This effectively gives us random draws from the joint unconditional distribution of $X_{t}$ within each model.

Now, we use the $N$ simulated samples of zero-coupon yields as input into a corresponding number of Kalman filter estimations. In each case, we use the true parameters as the starting point for each optimization. Since we are estimating the true model using the true parameters as the starting point for each optimization, this provides us with a clean read of the properties of the standard Kalman filter as an estimator, not impacted by any errors related to model misspecification.

In the final step, we use the $N$ simulated samples of zero-coupon yields as input into a corresponding number of Kalman filter estimations based on the DNS model, which is misspecified by construction. However, by comparing the results to those obtained from estimations of the true model that generated the data we get a very direct read of the potential bias involved in using the DNS model for yield extrapolations subject to various types of model misspecifications. First, by comparing to the CV model results, we can assess whether it matters to enforce theoretical consistency in the form of absence of arbitrage. Second, 
when we compare to the SV model results, we can further assess to what extent it matters to account for stochastic volatility in yields. Finally, the comparison to the GCV model results can speak to the importance of having the correct number of state variables.

\section{B.5 CV Model Results}

To begin, we analyze the results we get from the $N$ samples simulated based on the $\mathrm{CV}$ model. For each sample we perform three estimations, one based on the CV model using all 12 yields, one based on the CV model using only yields with maturities up to $\tau_{\max }$, and one based on the DNS model using only yields with maturities up to $\tau_{\max }$.

In Figure 1, each panel shows the mean fitted error of the 50-year yield from the three model estimations for all $N$ samples. First, we focus on the black lines, which are identical in all three panels and show the mean fitted errors for the 50-year yield when that yield is included in the model estimations. It is practically zero in all simulations, which shows that the CV model has no difficulty in fitting the 50-year yields in sample. Second, we note that when the maximum maturity used in the $\mathrm{CV}$ model estimation is reduced, the mean extrapolation error of the 50-year yield can fluctuate notably across samples as indicated by the variation in the solid blue lines. Still, it is the case that the fluctuations happen around the zero-line, so in expectation the mean fitted error is close to zero. Finally, it is noted that the mean fitted errors of the 50-year yield from the DNS model are downward biased, and more so the shorter the maximum yield maturity used in the model estimation, that is, the fitted 50-year yields implied by the DNS model are systematically above the simulated 50 -year yields. This bias arises thanks to the shape of the yield-adjustment term $\frac{A(\tau)}{\tau}$ in the CV model, which becomes increasingly negative as the maturity goes to infinity. Since the DNS model does not contain such a term, its extrapolated 50-year yield is essentially a flat extrapolation of the estimated value of the level factor, which is anchored near the level of the longest observed yield maturity. As a consequence, the fitted 50-year yield from the DNS model will naturally embed the effect from the yield-adjustment term when the maximum maturity of the observed yields approaches 50 years. This drives the bias towards zero as the longest yield maturity included in the model estimation approaches 50 years.

In terms of magnitude, the average of the mean extrapolation error of the fitted 50-year yields across the $N$ simulations is $-0.39 \%,-0.30 \%$, and $-0.24 \%$, when the maximum yield maturity used in the model estimation is 10 years, 20 years, and 30 years, respectively. Once 


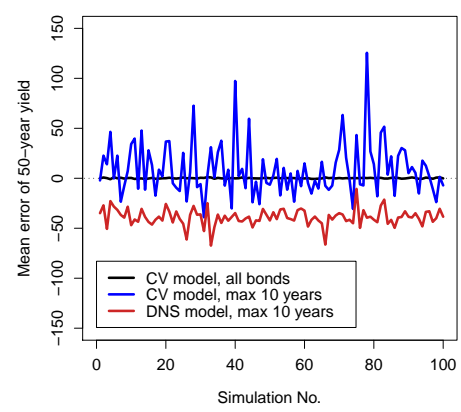

(a) 10-year maximum maturity

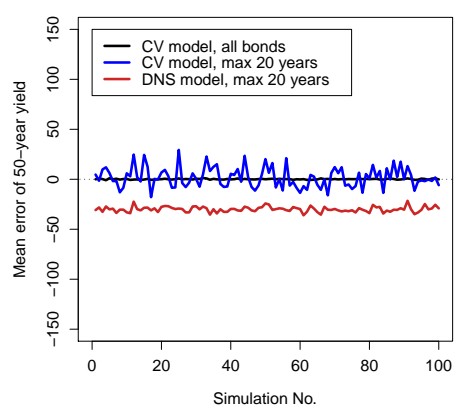

(b) 20-year maximum maturity

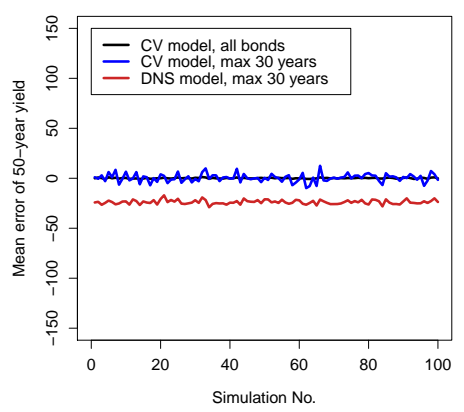

(c) 30-year maximum maturity

\section{Figure 1: Extrapolation Errors in CV Model Simulations}

Illustration of the mean fitted error of the simulated 50-year yield from the CV model. Each figure contains results from three model estimations: (1) the CV model estimated with all simulated bond yields, (2) the CV model estimated with a maximum maturity of the simulated yields as indicated, and (3) the DNS model estimated with a maximum maturity of the simulated yields as indicated.

this bias is compared with the general variation in yield levels, which is orders of magnitude larger as we demonstrate in Section 6 in the paper, it follows that the DNS model generates what must be considered reliable extrapolated 50-year yields, even if only yields with at most ten years to maturity are used in the model estimation.

\section{B.6 SV Model Results}

In this section, we analyze the results we get with the simulated samples from the SV model. As in the previous section, we perform three estimations for each sample, one based on the SV model using all 12 yields, one based on the SV model using only yields with maturities

up to a given $\tau_{\max }$, and one based on the DNS model using only yields with maturities up to the same given $\tau_{\max }$.

In Figure 2, each panel shows the mean fitted error of the 50-year yield from the three model estimations for all $N$ samples. In this case, it is striking that there is practically no bias in the extrapolated 50-year yields from the DNS model. The average of the mean fitted 50 -year yield extrapolation errors are $-0.04 \%,-0.03 \%$, and $-0.03 \%$, when the maximum yield maturity used in the model estimation is 10 years, 20 years, and 30 years, respectively. Thus, if the SV model happens to be the true model generating the yields we observe, relying on the DNS model for the calculation of extrapolated 50-year yields would imply almost no bias on average, even if only 10-year yields are used in the model estimation. This reinforces the results we got from the simulated yield samples based on the CV model. 


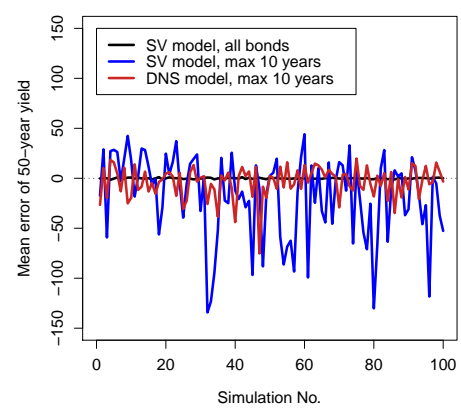

(a) 10-year maximum maturity

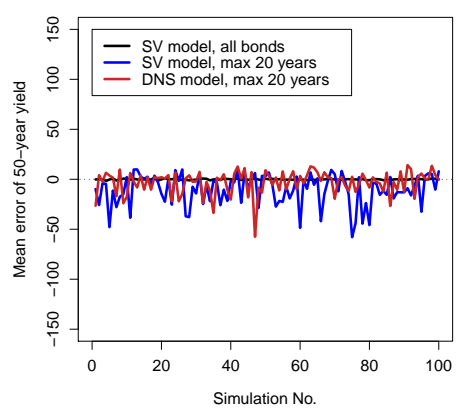

(b) 20-year maximum maturity

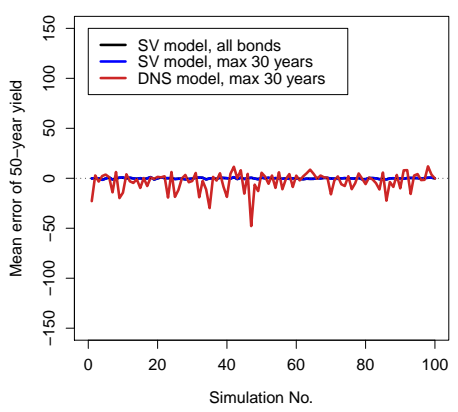

(c) 30-year maximum maturity

\section{Figure 2: Extrapolation Errors in SV Model Simulations}

Illustration of the mean fitted error of the simulated 50-year yield from the SV model. Each figure contains results from three model estimations: (1) the SV model estimated with all simulated bond yields, (2) the SV model estimated with a maximum maturity of the simulated yields as indicated, and (3) the DNS model estimated with a maximum maturity of the simulated yields as indicated.

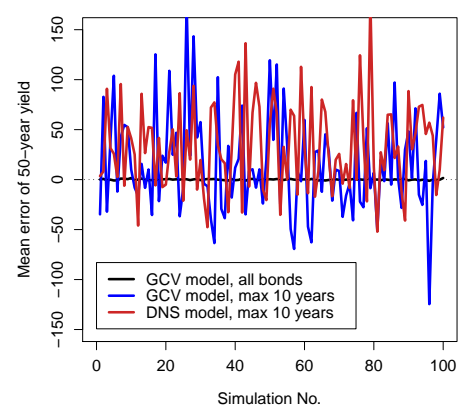

(a) 10-year maximum maturity

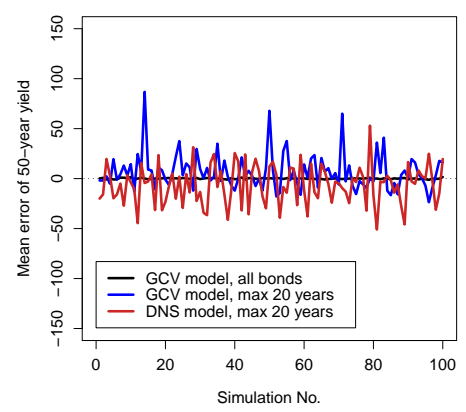

(b) 20-year maximum maturity

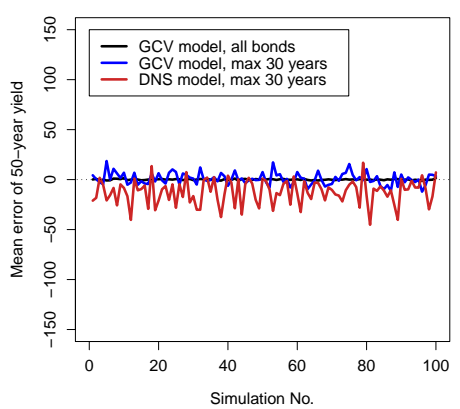

(c) 30-year maximum maturity

\section{Figure 3: Extrapolation Errors in GCV Model Simulations}

Illustration of the mean fitted error of the simulated 50-year yield from the GCV model. Each figure contains results from three model estimations: (1) the GCV model estimated with all simulated bond yields, (2) the CV model estimated with a maximum maturity of the simulated yields as indicated, and (3) the DNS model estimated with a maximum maturity of the simulated yields as indicated.

\section{B.7 GCV Model Results}

In this section, we analyze the results we get from the samples simulated based on the estimated GCV model. As in the previous section, we perform three estimations for each sample, one based on the GCV model using all 12 yields, one based on the GCV model using only yields with maturities up to a given $\tau_{\max }$, and one based on the DNS model using only yields with maturities up to the same given $\tau_{\max }$.

In Figure 3, each panel shows the mean fitted error of the 50-year yield from the three 
model estimations for all $N$ samples. With both 20 years and 30 years as the maximum maturity there is only modest bias in the 50-year yields extrapolated from the DNS model. Furthermore, they are only slightly larger and more volatile than the extrapolation errors observed for the true model. It is only if we limit the sample to yields with at most 10 years to maturity that the extrapolation errors start to be very erratic. Thus, we take these results to show that the DNS model is also able to generate reliable extrapolated long-term yields if the GCV model happens to be the model that generates the yields we observe.

In terms of magnitude, the average of the mean extrapolation error of the fitted 50-year yields across the $N$ simulated samples is $0.34 \%,-0.06 \%$, and $-0.13 \%$, when the maximum yield maturity used in the model estimation is 10 years, 20 years, and 30 years, respectively.

\section{B.8 Summary}

To summarize the results from the simulation study, we find that under realistic conditions where the DNS model is estimated with yields with maturities up to 30 years, it is able to produce extrapolated long bond yields with only a small bias, and this holds for all three arbitrage-free affine dynamic term structure models considered. Furthermore, even if only bonds of shorter maturity than thirty years are available, the DNS model will still produce extrapolated long bond yields with little bias in most cases.

\section{Additional Results for Canada}

In this appendix, we report additional results for the sample of Canadian government bond prices described in Section 4.1 of the paper.

Figure 4 shows the individual fitted error series for each bond's bond yield to maturity based on the DNS model estimated using all available bonds, while Tables 5 and 6 report the mean and RMSE for each of the 105 bonds in the sample.

\section{C.1 Interest Rate Scenarios Using the Canadian Data}

The estimated parameters of the unconstrained DNS model of Canadian government bond prices are reported in the last two columns of Table 4 in the paper. The model fit is reported in the last two columns of Table 5 of the paper. For all bonds combined, the RMSE is 6.81 basis points. 


\begin{tabular}{|c|c|c|c|c|c|c|}
\hline \multirow{2}{*}{ Bond } & \multicolumn{2}{|c|}{ Max 10 years } & \multicolumn{2}{|c|}{ Max 30 years } & \multicolumn{2}{|c|}{ Max 50 years } \\
\hline & Mean & RMSE & Mean & RMSE & Mean & RMSE \\
\hline (1) $10.5 \% 3 / 15 / 2021^{+}$ & 5.86 & 13.32 & 4.05 & 8.08 & 3.92 & 8.05 \\
\hline (2) $9.75 \% 6 / 1 / 2021^{+}$ & 3.69 & 11.48 & 1.87 & 5.53 & 1.74 & 5.52 \\
\hline (3) $9.25 \% 6 / 1 / 2022^{+}$ & 4.69 & 12.2 & 2.83 & 6.08 & 2.74 & 6.05 \\
\hline (4) $8 \% 6 / 1 / 2023^{+}$ & 4.46 & 10.96 & 2.62 & 4.71 & 2.58 & 4.68 \\
\hline (5) $9 \% 6 / 1 / 2025^{+}$ & 6.3 & 12.96 & 4.58 & 6.29 & 4.62 & 6.31 \\
\hline (6) $8 \% 6 / 1 / 2027^{+}$ & 4.12 & 13.42 & 2.6 & 5.71 & 2.74 & 5.82 \\
\hline (7) $5.75 \% 6 / 1 / 2029^{+}$ & -1.25 & 15.64 & -2.48 & 9.01 & -2.25 & 9.12 \\
\hline (8) $4.5 \% 6 / 1 / 2001$ & 1.97 & 3.9 & 1.9 & 3.67 & 1.83 & 3.6 \\
\hline (9) $5 \% 9 / 1 / 2004^{*}$ & 3.37 & 6.15 & 3.02 & 6.32 & 3.04 & 6.23 \\
\hline (10) $5.25 \% 12 / 1 / 2001$ & 4.6 & 7.76 & 3.9 & 6.92 & 3.81 & 6.82 \\
\hline (11) $5.5 \% 6 / 1 / 2010^{\dagger}$ & 1.58 & 5.24 & 0.86 & 5.99 & 0.84 & 5.94 \\
\hline (12) $6 \% 9 / 1 / 2005^{*}$ & 2.42 & 5.66 & 1.08 & 5.4 & 1.16 & 5.33 \\
\hline (13) $5.75 \% 6 / 1 / 2002$ & 0.41 & 9.45 & -0.06 & 9.63 & -0.17 & 9.55 \\
\hline (14) $6 \% 6 / 1 / 2011^{\dagger}$ & 1.24 & 3.54 & 0.8 & 5.39 & 0.77 & 5.35 \\
\hline (15) $6 \% 12 / 1 / 2002$ & -5.06 & 8.84 & -4.4 & 10.42 & -4.41 & 10.42 \\
\hline (16) $5.75 \% 9 / 1 / 2006^{*}$ & 2.26 & 3.9 & 1.04 & 4.2 & 1.14 & 4.2 \\
\hline (17) $5.75 \% 6 / 1 / 2003$ & -2.94 & 6.74 & -1.81 & 8.18 & -1.86 & 8.1 \\
\hline (18) $5 \% 12 / 1 / 2003$ & 0.19 & 6.88 & 1.14 & 9.15 & 1.04 & 8.9 \\
\hline (19) $5.75 \% 6 / 1 / 2033^{+}$ & -4.47 & 19.14 & -4.42 & 10.76 & -4.03 & 10.92 \\
\hline (20) $5.25 \% 6 / 1 / 2012^{\dagger}$ & 1.15 & 3.67 & 1.5 & 3.55 & 1.45 & 3.54 \\
\hline (21) $4.5 \% 9 / 1 / 2007^{*}$ & 1.78 & 3.75 & 0.78 & 3.63 & 0.85 & 3.65 \\
\hline (22) $3.5 \% 6 / 1 / 2004$ & 0.29 & 6.09 & 0.8 & 7.47 & 0.74 & 7.28 \\
\hline (23) $4.25 \% 12 / 1 / 2004$ & -1.93 & 3.34 & -2.17 & 4.24 & -2.11 & 4.15 \\
\hline (24) $5.25 \% 6 / 1 / 2013^{\dagger}$ & 0.84 & 4.01 & 0.45 & 3.5 & 0.4 & 3.55 \\
\hline (25) $4.25 \% 9 / 1 / 2008^{*}$ & 1.17 & 3.27 & 0.21 & 3.02 & 0.2 & 3.02 \\
\hline (26) $3.5 \% 6 / 1 / 2005$ & -4.58 & 6.16 & -5.21 & 7.23 & -5.11 & 7.05 \\
\hline (27) $3 \% 12 / 1 / 2005$ & -3.23 & 4.31 & -4.21 & 5.85 & -4.11 & 5.7 \\
\hline (28) $5 \% 6 / 1 / 2014^{\dagger}$ & 0.27 & 3.81 & -0.27 & 4.68 & -0.31 & 4.73 \\
\hline (29) $4.25 \% 9 / 1 / 2009$ & 1.08 & 4.98 & 0.68 & 4.92 & 0.64 & 4.84 \\
\hline (30) $3 \% 6 / 1 / 2006$ & -4.34 & 5.8 & -4.99 & 7.07 & -4.87 & 6.94 \\
\hline (31) $3.25 \% 12 / 1 / 2006$ & 0.28 & 3.02 & 0.64 & 3 & 0.72 & 3.01 \\
\hline (32) $5 \% 6 / 1 / 2037^{+}$ & -4.61 & 16.8 & -6.99 & 13.56 & -6.4 & 13.65 \\
\hline (33) $4.5 \% 6 / 1 / 2015^{\dagger}$ & -0.7 & 2.72 & -2.42 & 5.7 & -2.43 & 5.81 \\
\hline (34) $4 \% 9 / 1 / 2010^{*}$ & -1.78 & 5.29 & -1.73 & 5.25 & -1.68 & 5.27 \\
\hline (35) $3 \% 6 / 1 / 2007$ & 0.08 & 2.12 & 0.28 & 2.5 & 0.33 & 2.58 \\
\hline (36) $2.75 \% \quad 12 / 1 / 2007$ & -1.99 & 3.91 & -1.9 & 4.56 & -1.85 & 4.57 \\
\hline (37) $4 \% 6 / 1 / 2016^{\dagger}$ & -0.58 & 2.83 & -3.04 & 6.86 & -3.08 & 6.94 \\
\hline (38) $3.75 \% 9 / 1 / 2011^{*}$ & 1.28 & 3.26 & 1.3 & 3.73 & 1.35 & 3.77 \\
\hline (39) $3.75 \% 6 / 1 / 2008$ & -0.2 & 3.14 & -0.41 & 3.52 & -0.44 & 3.44 \\
\hline (40) $4.25 \% 12 / 1 / 2008$ & -0.81 & 3.86 & -1.54 & 5.05 & -1.55 & 5.13 \\
\hline (41) $4 \% 6 / 1 / 2017^{\dagger}$ & -0.53 & 3.05 & -3.37 & 7.05 & -3.51 & 7.18 \\
\hline (42) $3.75 \% 6 / 1 / 2012^{*}$ & 1.88 & 3.47 & 1.32 & 3.17 & 1.35 & 3.26 \\
\hline (43) $3.75 \%$ 6/1/2009 & -1.12 & 3.54 & -1.45 & 4.01 & -1.55 & 3.99 \\
\hline (44) $4.25 \% \quad 12 / 1 / 2009$ & 1.78 & 9.62 & 1.9 & 9.39 & 1.87 & 9.25 \\
\hline (45) $4.25 \% 6 / 1 / 2018^{\dagger}$ & 0.33 & 3.14 & -3.39 & 6.6 & -3.64 & 6.73 \\
\hline (46) $3.75 \% 6 / 1 / 2010$ & -1.74 & 6.29 & -0.73 & 6.48 & -0.66 & 6.36 \\
\hline (47) $3.5 \% 6 / 1 / 2013^{*}$ & 1.67 & 3.79 & -0.51 & 4.52 & -0.47 & 4.63 \\
\hline (48) $2.75 \% 12 / 1 / 2010$ & -6.26 & 9.1 & -5.44 & 9.32 & -5.36 & 9.33 \\
\hline (49) $4 \% 6 / 1 / 2041^{+}$ & -8.71 & 20.96 & -12.96 & 19.4 & -12 & 19.24 \\
\hline (50) $3.75 \% 6 / 1 / 2019^{\dagger}$ & 0.33 & 3.32 & -3.81 & 6.15 & -4.13 & 6.33 \\
\hline (51) $3 \% 6 / 1 / 2014^{*}$ & 0.56 & 2.88 & -0.64 & 5.44 & -0.61 & 5.51 \\
\hline (52) $1.25 \% 6 / 1 / 2011$ & -4.75 & 6.37 & -3.81 & 6.12 & -3.79 & 6.16 \\
\hline (53) $2 \% 12 / 1 / 2014^{*}$ & -0.72 & 2.03 & -1.6 & 4.9 & -1.5 & 4.96 \\
\hline (54) $1 \% 9 / 1 / 2011$ & -3.13 & 4.06 & -2.55 & 3.92 & -2.55 & 3.92 \\
\hline (55) $2 \% 9 / 1 / 2012$ & 1.89 & 4.64 & 1.18 & 5.32 & 1.24 & 5.46 \\
\hline
\end{tabular}

Table 5: Summary Statistics of Fitted Errors of Canadian Government Bond Yields

The mean fitted errors and the root mean-squared fitted errors (RMSE) of the yield to maturity for individual Canadian fixed-coupon government bonds according to the DNS model estimated on samples with varying maximum bond maturity. Asterisk * indicates five-year bonds, dagger $\dagger$ indicates ten-year bonds, plus + indicates thirty-year bonds, and cross $\times$ indicates fifty-year bonds. All numbers are measured in basis points. 


\begin{tabular}{|c|c|c|c|c|c|c|}
\hline \multirow{2}{*}{ Bond } & \multicolumn{2}{|c|}{ Max 10 years } & \multicolumn{2}{|c|}{ Max 30 years } & \multicolumn{2}{|c|}{ Max 50 years } \\
\hline & Mean & RMSE & Mean & $\overline{R M S E}$ & Mean & RMSE \\
\hline (56) $1.25 \% \quad 12 / 1 / 2011$ & -2.18 & 3.75 & -1.57 & 3.42 & -1.6 & 3.52 \\
\hline (57) $3.5 \% 6 / 1 / 2020^{\dagger}$ & 0.24 & 2.86 & -3.26 & 4.68 & -3.59 & 4.93 \\
\hline (58) $1.5 \% 3 / 1 / 2012$ & -1.72 & 3.28 & -1.35 & 3.4 & -1.39 & 3.57 \\
\hline (59) $2.5 \% 6 / 1 / 2015^{*}$ & -1.21 & 2.71 & -1.98 & 4.89 & -1.86 & 4.91 \\
\hline (60) $1.75 \% 3 / 1 / 2013$ & 0.7 & 3.04 & -0.62 & 5.13 & -0.53 & 5.29 \\
\hline (61) $1.5 \%$ 6/1/2012 & -1.33 & 3.74 & -1.08 & 4.4 & -1.1 & 4.56 \\
\hline (62) $3 \% 12 / 1 / 2015^{*}$ & -0.07 & 2.25 & -0.64 & 4.4 & -0.54 & 4.42 \\
\hline (63) $2.5 \% 9 / 1 / 2013$ & 1.14 & 2.17 & -0.08 & 3.08 & 0.02 & 3.19 \\
\hline (64) $3.25 \% 6 / 1 / 2021^{\dagger}$ & -0.68 & 2.47 & -3.92 & 5.32 & -4.22 & 5.58 \\
\hline (65) $1.5 \% 12 / 1 / 2012$ & -0.71 & 2.87 & -2.33 & 6.46 & -2.29 & 6.67 \\
\hline (66) $2 \% 6 / 1 / 2016^{*}$ & -1.09 & 3.1 & -1.74 & 5.61 & -1.68 & 5.56 \\
\hline (67) $2 \% 3 / 1 / 2014$ & 0.6 & 1.29 & 1.13 & 2.51 & 1.23 & 2.55 \\
\hline (68) $2 \% 8 / 1 / 2013$ & -0.69 & 2.91 & -2.1 & 5.88 & -2.02 & 6.07 \\
\hline (69) $2.75 \% 9 / 1 / 2016^{*}$ & 0.18 & 2.37 & -0.39 & 4.6 & -0.35 & 4.58 \\
\hline (70) $2.25 \% 8 / 1 / 2014$ & 0.21 & 2.12 & 1.51 & 3.46 & 1.63 & 3.57 \\
\hline (71) $3.5 \% 12 / 1 / 2045^{+}$ & -8.6 & 21.03 & -9.57 & 11.01 & -8.06 & 10.05 \\
\hline (72) $1.5 \% \quad 11 / 1 / 2013$ & -0.44 & 1.69 & -0.59 & 3.23 & -0.51 & 3.36 \\
\hline (73) $2.75 \% 6 / 1 / 2022^{\dagger}$ & 1.06 & 2.23 & -3.03 & 5.09 & -3.25 & 5.24 \\
\hline (74) $1.5 \% 3 / 1 / 2017^{*}$ & -1.18 & 3.17 & -1.26 & 4.84 & -1.32 & 4.88 \\
\hline (75) $1 \% 2 / 1 / 2014$ & -0.33 & 1.04 & 0.77 & 2.13 & 0.83 & 2.21 \\
\hline (76) $1 \% 2 / 1 / 2015$ & -0.6 & 1.69 & 0.74 & 2.99 & 0.94 & 3.05 \\
\hline (77) $0.75 \% 5 / 1 / 2014$ & -0.57 & 1.64 & 1.6 & 2.53 & 1.67 & 2.65 \\
\hline (78) $1.5 \% 8 / 1 / 2015$ & -0.69 & 3.29 & 0.02 & 5.55 & 0.23 & 5.51 \\
\hline (79) $1.5 \% 8 / 1 / 2017^{*}$ & 1.31 & 3.18 & 1.48 & 4.31 & 1.32 & 4.29 \\
\hline (80) $1 \% 11 / 1 / 2014$ & 0.01 & 1.19 & 1.86 & 2.83 & 2.03 & 2.96 \\
\hline (81) $1.5 \% 6 / 1 / 2023^{\dagger}$ & -0.01 & 3.23 & -4.2 & 5.47 & -4.26 & 5.52 \\
\hline (82) $1.25 \% 2 / 1 / 2016$ & -1.03 & 3.48 & -0.99 & 5.64 & -0.79 & 5.52 \\
\hline (83) $1.25 \% 3 / 1 / 2018^{*}$ & 0.71 & 3.59 & 0.7 & 4.51 & 0.43 & 4.53 \\
\hline (84) $1 \% 5 / 1 / 2015$ & -1.24 & 2.65 & -1.01 & 3.15 & -0.78 & 3.09 \\
\hline (85) $1 \% 8 / 1 / 2016$ & -1.52 & 3.81 & -2.34 & 6.7 & -2.2 & 6.56 \\
\hline (86) $1.25 \%$ 9/1/2018* & 0.56 & 4.18 & 0.38 & 4.55 & 0.02 & 4.61 \\
\hline (87) $2.5 \% 6 / 1 / 2024^{\dagger}$ & 0.28 & 2.33 & -1.64 & 4.65 & -1.44 & 4.51 \\
\hline (88) $1 \% 11 / 1 / 2015$ & -1.8 & 3.84 & -2.47 & 4.67 & -2.24 & 4.48 \\
\hline (89) $1.5 \% 2 / 1 / 2017$ & 0.74 & 2.51 & 1.18 & 4.26 & 1.16 & 4.24 \\
\hline (90) $1.75 \% 3 / 1 / 2019^{*}$ & -0.18 & 4.29 & -0.31 & 4.57 & -0.78 & 4.73 \\
\hline (91) $1 \% 5 / 1 / 2016$ & -0.91 & 4.04 & -1.3 & 6.32 & -1.17 & 6.21 \\
\hline (92) $1.75 \%$ 9/1/2019* & -0.43 & 3.89 & -0.78 & 4.44 & -1.32 & 4.65 \\
\hline (93) $2.75 \% 12 / 1 / 2064^{\times}$ & -21.34 & 26.26 & -22.86 & 23.25 & -19.56 & 19.94 \\
\hline (94) $1.25 \% 8 / 1 / 2017$ & 2.41 & 3.33 & 4.45 & 5.53 & 4.24 & 5.43 \\
\hline (95) $2.75 \% 12 / 1 / 2048^{+}$ & -7.27 & 16.27 & -10.02 & 10.53 & -7.02 & 7.67 \\
\hline (96) $2.25 \% 6 / 1 / 2025^{\dagger}$ & 2.1 & 2.83 & -3.37 & 5.45 & -2.79 & 5.17 \\
\hline (97) $1 \% 11 / 1 / 2016$ & -0.51 & 2.81 & 0.2 & 4.22 & 0.27 & 4.15 \\
\hline (98) $1.5 \% 3 / 1 / 2020^{*}$ & -1.65 & 4.95 & -2.61 & 5.29 & -3.19 & 5.65 \\
\hline (99) $1.25 \% 2 / 1 / 2018$ & 1.73 & 2.84 & 4.34 & 6.17 & 4.02 & 6.02 \\
\hline (100) $0.25 \% 5 / 1 / 2017$ & 2.69 & 2.95 & 4.89 & 5.34 & 4.85 & 5.30 \\
\hline (101) $0.75 \% 9 / 1 / 2020$ & -3.04 & 5.4 & -5.1 & 6.01 & -5.7 & 6.54 \\
\hline (102) $1.5 \% 6 / 1 / 2026^{\dagger}$ & 4.56 & 4.66 & -6.24 & 6.92 & -5.4 & 6.23 \\
\hline (103) $0.25 \% \quad 11 / 1 / 2017$ & 3.86 & 3.98 & 7.99 & 8.14 & 7.85 & 8.02 \\
\hline (104) $0.75 \% 3 / 1 / 2021^{*}$ & -3.98 & 5.17 & -6.32 & 6.7 & -6.88 & 7.24 \\
\hline (105) $0.25 \% 5 / 1 / 2018$ & 3.79 & 3.81 & 9.92 & 9.97 & 9.68 & 9.75 \\
\hline All yields & 0.38 & 9.02 & -0.75 & 6.86 & -0.68 & 6.81 \\
\hline Max $\log L$ & \multicolumn{2}{|c|}{$25,052.23$} & \multicolumn{2}{|c|}{$33,282.71$} & \multicolumn{2}{|c|}{ 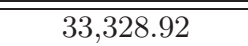 } \\
\hline
\end{tabular}

Table 6: Summary Statistics of Fitted Errors of Canadian Government Bond Yields cont.

The mean fitted errors and the root mean-squared fitted errors (RMSE) of the yield to maturity for individual Canadian fixed-coupon government bonds according to the DNS model estimated on samples with varying maximum bond maturity. Asterisk * indicates five-year bonds, dagger $\dagger$ indicates ten-year bonds, plus + indicates thirty-year bonds, and cross $\times$ indicates fifty-year bonds (also highlighted in bold). All numbers are measured in basis points. 


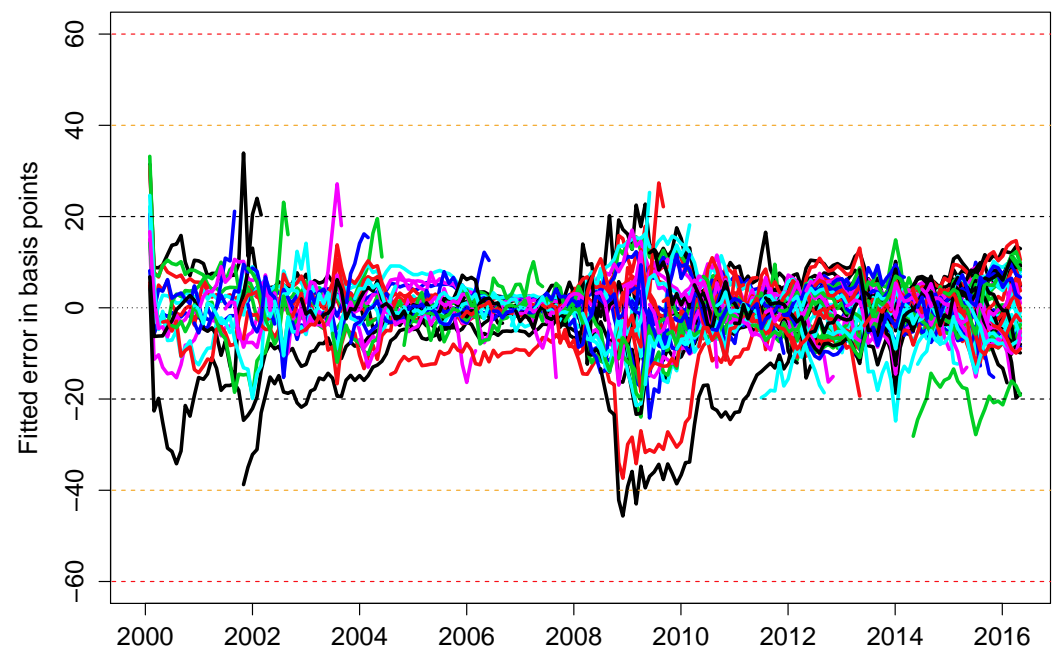

Figure 4: Fitted Errors from the DNS Model of Canadian Government Bond Prices Illustration of the fitted errors of Canadian government fixed-coupon bond yields to maturity implied by the DNS model estimated with all Canadian government fixed-coupon bond prices. The bond price series are monthly and cover the period from January 31, 2000, to April 29, 2016.

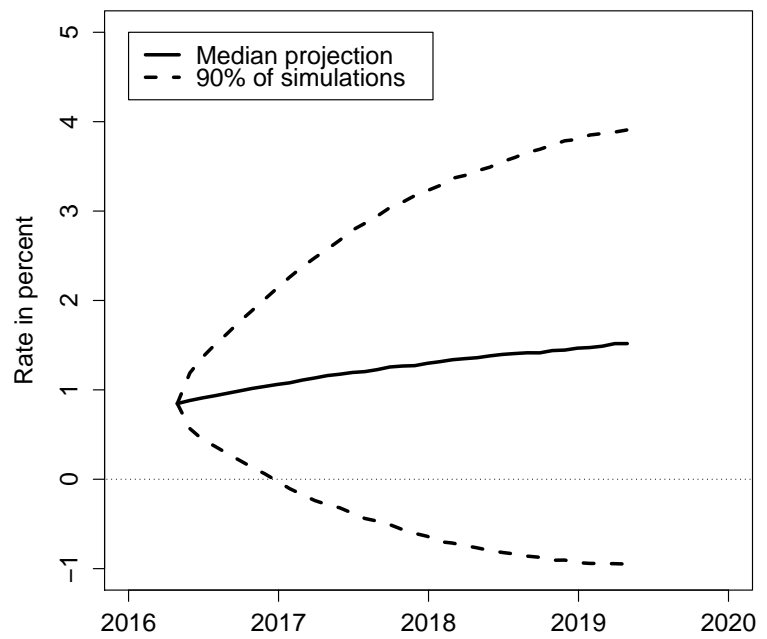

Figure 5: Short-Rate Projections from the Canadian DNS Model

Illustration of the median and [5\%,95\%] range of the short rate from the Canadian DNS model's simulated interest rate scenarios as of April 29, 2016. 
As in the Swiss and U.S. cases described in the paper, we simulate 10,000 factor paths over a three-year horizon using as conditioning information the estimated parameters and factor values as of April 29, 2016. To keep things simple, we focus on the simulated paths for the short rate, which is the model equivalent for the overnight rate targeted by the Bank of Canada in its implementation of monetary policy, and on the projected ten-year Canadian government bond yield, which is a widely used benchmark for many fixed-income securities, such as MBS.

To provide insight into the variation in the DNS model outputs, Figure 5 shows projections of the short rate as of April 29, 2016. The figure shows the median, 5th, and 95th percentile values for the simulated short rate for a three-year forecast horizon. The median simulated short rate indicates a continued gradual normalization of monetary policy with the short rate reaching a level around 1.5 percent by mid-2019. The upper 95 th percentile rises more rapidly and reaches 4 percent at three years out, while the lower 5 th percentile represents a movement into negative policy rates in early 2017. Furthermore, note the width of the [5\%,95\%] range. By mid-2019, there is a 500 basis point difference between the top and the bottom of this range.

Next, we focus on the simulated paths for the ten-year Canadian government bond yield. Figure 6 illustrates the median and [5\%,95\%] range of the ten-year yield at each point in time from the simulated interest rate scenarios. The median simulated ten-year yield indicates a steady, very gradual normalization of the long-term interest rate environment. The upper 95th percentile rises more rapidly and reaches 4 percent at three years out, while the lower 5th percentile represents a further decline from the already very low current interest rate environment. To provide perspective for the model projections, we compare them to the Consensus Forecasts survey performed on May 9, 2016. It contains 15 responses to questions about the level of the ten-year Canadian government bond yield at the end of August 2016 and the end of May 2017, respectively. In Figure 6, we show the consensus forecasts as well as the maximum and minimum response to those two questions. Note that the model-implied median projection is very close to the path of the consensus forecast path. Furthermore, the $[5 \%, 95 \%]$ range of the projected ten-year yields encompasses the range of the survey forecasts. More importantly, the projections suggest that the likely range of outcomes at a three-year horizon is quite wide at more than 300 basis points, which is only marginally smaller than the corresponding range of projected short rates. 


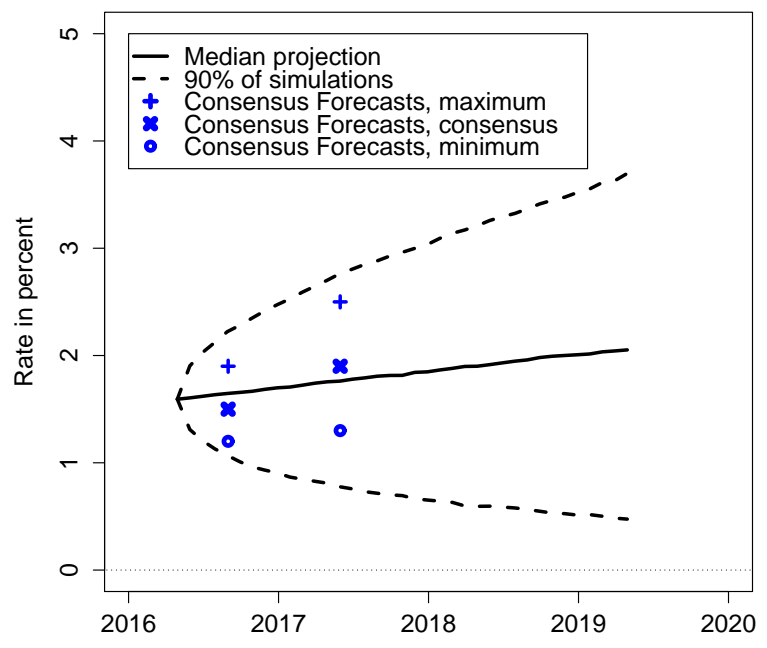

Figure 6: Ten-Year Yield Projections from the Canadian DNS Model

Illustration of the median and [5\%,95\%] range of the ten-year yield from the Canadian DNS model's simulated interest rate scenarios as of April 29, 2016. Also shown are the consensus forecasts as well as the maximum and minimum responses from the Consensus Forecasts survey performed on May 9, 2016.

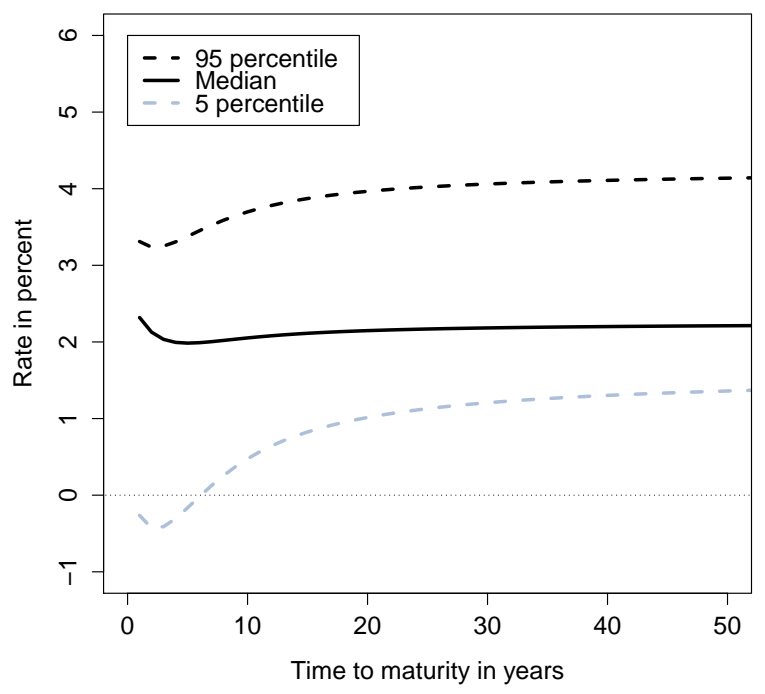

Figure 7: Simulated Canadian Yield Curves for April 2019

In Figure 7, we illustrate the entire yield curve corresponding to the three percentiles of the ten-year yield projections for the end of April 2019 shown in Figure 6. Note the wide variation 
in the shape of the yield curve that the model suggests may occur with $90 \%$ probability over the next three years.

\section{Additional Results for France}

In this appendix, we report additional results for the sample of French government bond prices described in Section 4.2 of the paper.

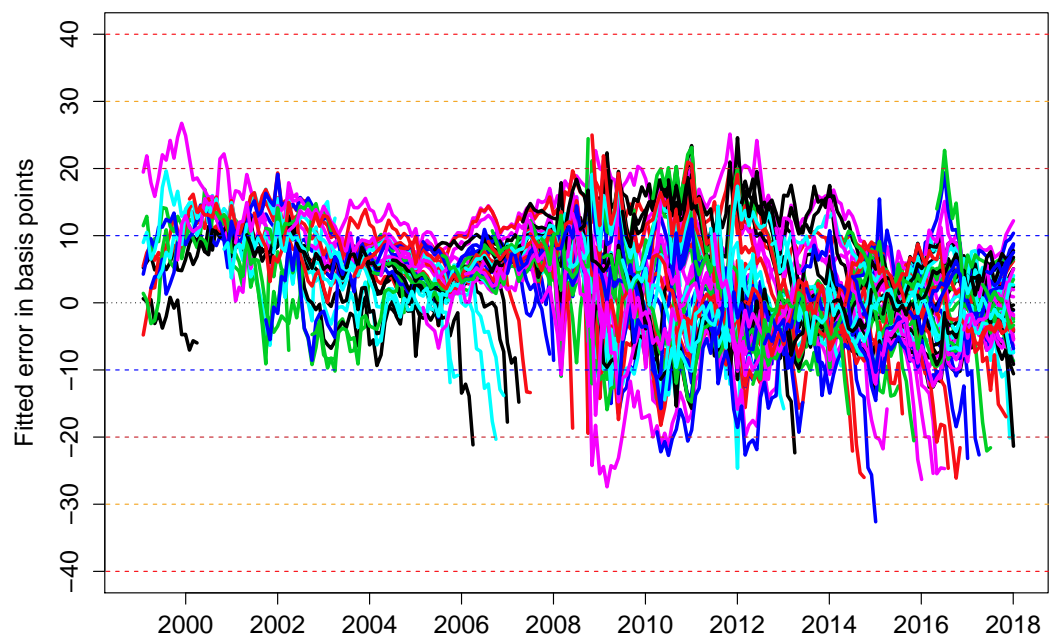

Figure 8: Fitted Errors from the DNS Model of French Government Bond Prices

Illustration of the fitted errors of French government fixed-coupon bond yields to maturity implied by the DNS model estimated with all French government fixed-coupon bond prices. The data are monthly and cover the period from January 29, 1999, to December 29, 2017.

Figure 8 shows the individual fitted error series for each bond's bond yield to maturity based on the DNS model estimated using all available bonds, while Tables 7 and 8 report the mean and RMSE for each of the 114 bonds in the sample.

\section{D.1 Interest Rate Scenarios Using the French Data}

The estimated parameters of the unconstrained DNS model of French government bond prices are reported in the last two columns of Table 6 in the paper. Its model fit is reported in the last two columns of Table 7. For all bonds combined, the RMSE is 8.01 basis points.

We simulate 10,000 factor paths over a three-year horizon using as conditioning informa- 


\begin{tabular}{|c|c|c|c|c|c|c|c|c|}
\hline \multirow{2}{*}{ Bond } & \multicolumn{2}{|c|}{ Max 10 years } & \multicolumn{2}{|c|}{ Max 15 years } & \multicolumn{2}{|c|}{ Max 30 years } & \multicolumn{2}{|c|}{ Max 50 years } \\
\hline & Mean & RMSE & Mean & RMSE & Mean & RMSE & Mean & RMSE \\
\hline (1) $5.5 \% 4 / 25 / 2007$ & 5.75 & 7.22 & 5.47 & 7.12 & 4.96 & 6.71 & 4.94 & 6.66 \\
\hline (2) $5.5 \% 10 / 25 / 2007$ & 6.77 & 8.19 & 6.62 & 8.25 & 6.14 & 7.81 & 6.1 & 7.77 \\
\hline (3) $4.5 \% 7 / 12 / 2002$ & 6.55 & 8.29 & 6.7 & 8.41 & 8.18 & 9.92 & 8.15 & 9.94 \\
\hline (4) $5.25 \% 4 / 25 / 2008$ & 8.31 & 9.22 & 8.34 & 9.33 & 7.93 & 8.86 & 7.87 & 8.82 \\
\hline (5) $4.5 \% 7 / 12 / 2003$ & 7.36 & 8.91 & 7.33 & 9.06 & 8.59 & 10.58 & 8.54 & 10.53 \\
\hline (6) $5.5 \% 4 / 25 / 2029^{+}$ & 7.46 & 16.71 & 7.47 & 13.14 & 11.27 & 12.6 & 12.5 & 14.11 \\
\hline (7) $4 \% 7 / 12 / 2000$ & -0.01 & 3.27 & 0.05 & 3.27 & -2.49 & 3.72 & -2.47 & 3.6 \\
\hline (8) $4 \% 4 / 25 / 2009$ & 7.32 & 8.83 & 7.65 & 9.14 & 7.39 & 8.93 & 7.32 & 8.91 \\
\hline (9) $3.5 \% \quad 7 / 12 / 2004$ & 3.42 & 7.29 & 3.14 & 7.26 & 3.36 & 7.74 & 3.32 & 7.71 \\
\hline (10) $3 \% 7 / 12 / 2001$ & 7.52 & 8.35 & 7.76 & 8.68 & 8.16 & 8.81 & 8.32 & 9.01 \\
\hline (11) $4 \%$ 10/25/2009 & 6.77 & 8.76 & 7.26 & 9.24 & 7.33 & 9.13 & 7.29 & 9.11 \\
\hline (12) $4 \% 1 / 12 / 2002$ & 8.55 & 9.32 & 9.13 & 9.9 & 9.64 & 10.45 & 9.68 & 10.52 \\
\hline (13) $5 \% 7 / 12 / 2005$ & 3.75 & 7.73 & 3.38 & 7.43 & 3.02 & 7.28 & 3.03 & 7.24 \\
\hline (14) $5.5 \% \quad 4 / 25 / 2010$ & 6.27 & 8.84 & 6.8 & 9.45 & 7.18 & 9.73 & 7.12 & 9.67 \\
\hline (15) $5 \% 1 / 12 / 2003$ & 3.27 & 6.49 & 3.24 & 6.58 & 3.93 & 7.39 & 3.92 & 7.44 \\
\hline (16) $5.5 \% \quad 10 / 25 / 2010$ & 4.76 & 7.81 & 5.46 & 8.53 & 6.07 & 9.16 & 6.02 & 9.16 \\
\hline (17) $5 \% 1 / 12 / 2006$ & 4.04 & 7.43 & 3.66 & 7.09 & 3.27 & 6.68 & 3.35 & 6.68 \\
\hline (18) $5 \% 10 / 25 / 2016^{*}$ & 3.38 & 6.34 & 3.94 & 6.47 & 4.47 & 7.92 & 4.37 & 8.25 \\
\hline (19) $4.5 \% 7 / 12 / 2006$ & 2.13 & 6.73 & 1.57 & 6.67 & 1.08 & 6.55 & 1.18 & 6.5 \\
\hline (20) $5.75 \% 10 / 25 / 2032^{+}$ & 1.79 & 17.74 & 1.39 & 11.84 & 7.15 & 8.38 & 8.67 & 10.06 \\
\hline (21) $5 \%$ 10/25/2011 & 3.78 & 6.51 & 4.38 & 7.22 & 4.78 & 8.23 & 4.7 & 8.27 \\
\hline (22) $4 \% 1 / 12 / 2004$ & 1.22 & 5.19 & 1.21 & 5.5 & 0.84 & 5.19 & 0.76 & 5.11 \\
\hline (23) $3.75 \% 1 / 12 / 2007$ & 2.52 & 6.14 & 1.96 & 6.17 & 1.5 & 6.3 & 1.58 & 6.28 \\
\hline (24) $5 \% 4 / 25 / 2012$ & 3.88 & 6.73 & 4.22 & 7.28 & 4.25 & 8.04 & 4.11 & 8.05 \\
\hline (25) $4.75 \% 5 / 16 / 2002$ & 3.74 & 5.56 & 3.34 & 5.35 & 3.03 & 5.22 & 3.06 & 5.22 \\
\hline (26) $4.75 \% \quad 10 / 25 / 2012$ & 3.79 & 6.56 & 4.04 & 6.97 & 3.9 & 7.47 & 3.75 & 7.52 \\
\hline (27) $3.5 \% 1 / 12 / 2005$ & -2.99 & 4.38 & -3.01 & 4.85 & -3.34 & 5.05 & -3.34 & 4.96 \\
\hline (28) $3.5 \% 1 / 12 / 2008$ & 4.97 & 5.5 & 4.72 & 5.43 & 4.67 & 5.54 & 4.67 & 5.55 \\
\hline (29) $4 \% 4 / 25 / 2013$ & 2.65 & 7.03 & 2.7 & 7.22 & 2.14 & 7.42 & 1.93 & 7.5 \\
\hline (30) $4.25 \% \quad 4 / 25 / 2019^{*}$ & 3.8 & 6.6 & 4.5 & 6.29 & 5.65 & 7.78 & 6.17 & 8.5 \\
\hline (31) $3 \% 7 / 12 / 2008$ & 5.62 & 6.21 & 5.68 & 6.41 & 5.81 & 6.57 & 5.8 & 6.63 \\
\hline (32) $4 \%$ 10/25/2013 & 3.08 & 6.22 & 2.84 & 6.52 & 1.94 & 6.66 & 1.66 & 6.8 \\
\hline (33) $3.5 \%$ 1/12/2009 & 5.08 & 6.23 & 5.12 & 6.48 & 5.22 & 6.71 & 5.16 & 6.65 \\
\hline (34) $4 \% 4 / 25 / 2014$ & 2.83 & 6.04 & 2.48 & 6.19 & 1.6 & 6.24 & 1.33 & 6.43 \\
\hline (35) $2.25 \% 3 / 12 / 2006$ & -1.67 & 3.5 & -1.68 & 3.94 & -1.72 & 4.41 & -1.53 & 4.33 \\
\hline (36) $4.75 \% 4 / 25 / 2035^{+}$ & -0.5 & 21.54 & -2.14 & 14.85 & 3.83 & 5.97 & 5.76 & 7.11 \\
\hline (37) $3.5 \% 7 / 12 / 2009$ & 4.96 & 5.79 & 5.24 & 6.06 & 5.78 & 6.6 & 5.76 & 6.64 \\
\hline (38) $4 \%$ 10/25/2014 & 3.09 & 5.92 & 2.58 & 5.88 & 1.63 & 5.95 & 1.37 & 6.15 \\
\hline (39) $3 \% 1 / 12 / 2010$ & 3.1 & 6.34 & 3.43 & 6.66 & 4.1 & 7.12 & 4.06 & 7.08 \\
\hline (40) $3.5 \% \quad 4 / 25 / 2015$ & 2.76 & 6.13 & 2.03 & 6.27 & 0.89 & 6.83 & 0.63 & 7.03 \\
\hline (41) $2.25 \% 3 / 12 / 2007$ & -0.28 & 4.29 & -0.79 & 4.67 & -1.29 & 5.37 & -1.22 & 5.29 \\
\hline (42) $4 \% 4 / 25 / 2055^{\times}$ & -10.95 & 35.72 & -14.11 & 29.18 & -6.07 & 14.31 & -3.59 & 10.56 \\
\hline (43) $3.75 \% 4 / 25 / 2021^{*}$ & 3.48 & 8.53 & 3.85 & 6.99 & 5.74 & 9.04 & 6.71 & 10.2 \\
\hline (44) $2.5 \% 7 / 12 / 2010$ & 1.45 & 6.27 & 1.79 & 6.66 & 2.55 & 7.18 & 2.5 & 7.19 \\
\hline (45) $3 \%$ 10/25/2015 & 2.42 & 5.23 & 1.72 & 4.87 & 0.62 & 4.97 & 0.33 & 5.29 \\
\hline (46) $2.75 \% 3 / 12 / 2008$ & 5.07 & 6.2 & 5.13 & 6.46 & 5.26 & 6.96 & 5.3 & 6.93 \\
\hline (47) $3 \% 1 / 12 / 2011$ & 1.74 & 6.45 & 2.26 & 6.61 & 3.07 & 7.36 & 3.13 & 7.55 \\
\hline (48) $3.25 \%$ 4/25/2016 & 2.92 & 5.75 & 2.38 & 5.51 & 1.5 & 5.82 & 1.25 & 6.1 \\
\hline (49) $3.5 \% 7 / 12 / 2011$ & 2.2 & 6.17 & 2.63 & 6.61 & 3.13 & 7.73 & 3.15 & 8.04 \\
\hline (50) $3.5 \% 9 / 12 / 2008$ & 5.68 & 6.94 & 5.82 & 7.6 & 5.81 & 8.29 & 5.88 & 8.37 \\
\hline (51) $4 \% 10 / 25 / 2038^{+}$ & -6.7 & 28.79 & -9.07 & 21.8 & -1.25 & 7.9 & 1.08 & 5.94 \\
\hline (52) $3.75 \% 4 / 25 / 2017$ & 2.67 & 5.44 & 2.85 & 5.6 & 2.79 & 6.73 & 2.63 & 7.25 \\
\hline (53) $3.75 \%$ 1/12/2012 & 1.88 & 6.89 & 1.9 & 7.1 & 1.52 & 7.68 & 1.37 & 7.7 \\
\hline (54) $4 \% 9 / 12 / 2009$ & 3.14 & 7.98 & 3.58 & 8.35 & 4.52 & 8.86 & 4.7 & 8.97 \\
\hline (55) $4.25 \% 10 / 25 / 2023^{*}$ & 1.63 & 10.47 & 2.02 & 7.84 & 5.56 & 10.84 & 6.9 & 12.42 \\
\hline (56) $4.5 \% 7 / 12 / 2012$ & 2.14 & 6.63 & 1.99 & 6.8 & 1.43 & 7.32 & 1.22 & 7.49 \\
\hline (57) $4.25 \% \quad 10 / 25 / 2017$ & 2.24 & 5.24 & 2.89 & 5.73 & 3.42 & 7.26 & 3.42 & 7.78 \\
\hline (58) $3.75 \%$ 1/12/2013 & 1.36 & 7.05 & 1.13 & 6.89 & 0.32 & 7.02 & 0.04 & 7.15 \\
\hline
\end{tabular}

Table 7: Summary Statistics of Fitted Errors of French Government Bond Yields The mean fitted errors and the root mean-squared fitted errors (RMSE) of the yield to maturity for individual French fixed-coupon government bonds according to the DNS model estimated on samples with varying maximum bond maturity. Asterisk * indicates fifteen-year bonds, dagger $\dagger$ indicates twenty-year bonds, plus + indicates thirty-year bonds, and cross $x$ indicates fifty-year bonds (also highlighted in bold). All numbers are measured in basis points. 


\begin{tabular}{|c|c|c|c|c|c|c|c|c|}
\hline \multirow{2}{*}{ Bond } & \multicolumn{2}{|c|}{ Max 10 years } & \multicolumn{2}{|c|}{ Max 15 years } & \multicolumn{2}{|c|}{ Max 30 years } & \multicolumn{2}{|c|}{ Max 50 years } \\
\hline & Mean & RMSE & Mean & RMSE & Mean & RMSE & Mean & RMSE \\
\hline (59) $4 \% 4 / 25 / 2018$ & 1.71 & 4.5 & 2.55 & 5.07 & 3.52 & 7.23 & 3.66 & 7.92 \\
\hline (60) $3.75 \%$ 9/12/2010 & -1.96 & 6.67 & -1.53 & 7.14 & -0.38 & 8.23 & -0.19 & 8.63 \\
\hline (61) $4.5 \%$ 7/12/2013 & -0.65 & 7.55 & -1.18 & 7.72 & -2.45 & 8.09 & -2.89 & 8.25 \\
\hline (62) $4.25 \% \quad 10 / 25 / 2018$ & 1.76 & 4.04 & 3.03 & 5.2 & 4.75 & 7.9 & 5.13 & 8.65 \\
\hline (63) $2.5 \%$ 1/12/2014 & -0.84 & 5.17 & -1.62 & 5.59 & -3.09 & 6.17 & -3.67 & 6.55 \\
\hline (64) $1.5 \% 9 / 12 / 2011$ & -2.34 & 4.1 & -1.83 & 4.66 & -1.25 & 6.22 & -0.89 & 6.82 \\
\hline (65) $3 \%$ 7/12/2014 & -0.42 & 4.59 & -1.17 & 4.72 & -2.45 & 5.05 & -3.01 & 5.47 \\
\hline (66) $4.5 \% 4 / 25 / 2041^{+}$ & -9.16 & 30.13 & -11.67 & 22.8 & -1.86 & 6.9 & 0.59 & 3.78 \\
\hline (67) $3.75 \%$ 10/25/2019 & 0.53 & 3.6 & 1.92 & 4.83 & 4.08 & 7.97 & 4.73 & 8.86 \\
\hline (68) $2.5 \% 1 / 15 / 2015$ & -0.23 & 5.95 & -1.16 & 6.51 & -2.65 & 7.31 & -3.25 & 7.47 \\
\hline (69) $3.5 \% 4 / 25 / 2020$ & 0.27 & 3 & 1.77 & 4.6 & 3.98 & 8.2 & 4.71 & 9.22 \\
\hline (70) $4 \% 4 / 25 / 2060^{\times}$ & -13.36 & 41.61 & -17.28 & 33.90 & -6.29 & 16.31 & -3.62 & 11.53 \\
\hline (71) $0.75 \% 9 / 20 / 2012$ & -2.9 & 4.08 & -3.08 & 4.15 & -2.84 & 4.99 & -2.66 & 5.62 \\
\hline (72) $2 \% 7 / 12 / 2015$ & -0.28 & 4.61 & -1.25 & 5.43 & -2.92 & 7.01 & -3.62 & 7.47 \\
\hline (73) $3.5 \% 4 / 25 / 2026^{*}$ & 1.95 & 11.08 & 1.53 & 5.69 & 5.5 & 8.4 & 6.88 & 10.71 \\
\hline (74) $2.5 \% \quad 10 / 25 / 2020$ & -1.08 & 2.7 & -0.06 & 3.81 & 1.56 & 6.73 & 2.25 & 7.6 \\
\hline (75) $2.25 \% 2 / 25 / 2016$ & 0.06 & 4.03 & -0.53 & 4.6 & -1.69 & 5.67 & -2.31 & 6.07 \\
\hline (76) $2 \% 9 / 25 / 2013$ & -4.11 & 6.81 & -4.49 & 7.04 & -4.69 & 7.2 & -4.74 & 7.04 \\
\hline (77) $3.25 \% 10 / 25 / 2021$ & -1.13 & 2.72 & -0.84 & 3.45 & 0.3 & 6.07 & 0.96 & 6.8 \\
\hline (78) $2.5 \% 7 / 25 / 2016$ & 0.04 & 4.14 & -0.45 & 5.04 & -1.56 & 6.7 & -2.17 & 7.2 \\
\hline (79) $3 \% 4 / 25 / 2022$ & -0.81 & 3.71 & -1 & 4.22 & -0.22 & 6.92 & 0.35 & 7.57 \\
\hline (80) $1.75 \% 2 / 25 / 2017$ & -0.36 & 3.73 & -0.43 & 4.52 & -1.25 & 6.52 & -1.96 & 7.55 \\
\hline (81) $0.75 \%$ 9/25/2014 & -3.08 & 5.02 & -3.59 & 5.36 & -3.63 & 5.87 & -3.64 & 5.95 \\
\hline (82) $1 \% 7 / 25 / 2017$ & -1.01 & 3.88 & -0.66 & 4.23 & -0.87 & 5.61 & -1.3 & 6.19 \\
\hline (83) $2.75 \% 10 / 25 / 2027^{*}$ & 7.96 & 11.27 & 3.51 & 5.64 & 3.66 & 5.29 & 3.93 & 6.55 \\
\hline (84) $2.25 \% \quad 10 / 25 / 2022$ & -0.15 & 4.19 & -1.34 & 4.57 & -1.84 & 6.63 & -1.56 & 6.9 \\
\hline (85) $1 \% 5 / 25 / 2018$ & -0.13 & 2.91 & 0.46 & 3.43 & 0.79 & 5.24 & 0.69 & 5.93 \\
\hline (86) $0.25 \% 11 / 25 / 2015$ & -1.15 & 3.33 & -1.69 & 4.3 & -2.3 & 5.79 & -2.58 & 6.14 \\
\hline (87) $3.25 \% 5 / 25 / 2045^{+}$ & 11.1 & 22.65 & 0.56 & 15.87 & 2.65 & 6.91 & 2.82 & 4.75 \\
\hline (88) $1.75 \% 5 / 25 / 2023$ & -1.14 & 3.4 & -2.99 & 4.43 & -4.14 & 6.88 & -3.99 & 7.11 \\
\hline (89) $1 \% 11 / 25 / 2018$ & 0.32 & 2.13 & 1.05 & 2.43 & 1.93 & 3.97 & 2.15 & 4.64 \\
\hline (90) $2.25 \% 5 / 25 / 2024$ & 0.44 & 4.22 & -2.23 & 4.34 & -4.18 & 7.43 & -4.24 & 7.92 \\
\hline (91) $1 \% 5 / 25 / 2019$ & 0.06 & 1.85 & 0.89 & 2.17 & 2.17 & 3.71 & 2.66 & 4.53 \\
\hline (92) $0.25 \% \quad 11 / 25 / 2016$ & -1.5 & 4.79 & -1.74 & 6.14 & -1.92 & 8.75 & -2.41 & 9.85 \\
\hline (93) $2.5 \% 5 / 25 / 2030^{*}$ & 12.33 & 14.25 & 4.84 & 5.83 & 1.3 & 3.35 & 0.51 & 4.56 \\
\hline (94) $1.75 \% \quad 11 / 25 / 2024$ & 1.74 & 4.23 & -1.69 & 2.9 & -4.93 & 5.85 & -5.31 & 6.31 \\
\hline (95) $0.5 \% 11 / 25 / 2019$ & -0.78 & 1.73 & -0.01 & 1.78 & 1.33 & 3.24 & 2 & 4.15 \\
\hline (96) $0.5 \% 5 / 25 / 2025$ & 1.35 & 3.02 & -1.89 & 2.44 & -5.32 & 5.91 & -5.98 & 6.6 \\
\hline (97) $0 \% 5 / 25 / 2020$ & -0.31 & 1.31 & 0.39 & 1.37 & 1.7 & 2.78 & 2.54 & 3.74 \\
\hline (98) $0 \% 2 / 25 / 2018$ & -0.68 & 4.01 & -0.23 & 4.68 & 0.46 & 6.63 & 0.53 & 7.38 \\
\hline (99) $1 \% 11 / 25 / 2025$ & 3.6 & 4.89 & 0.27 & 2.16 & -3.35 & 4.65 & -4.14 & 5.41 \\
\hline (100) $0.25 \% \quad 11 / 25 / 2020$ & -0.9 & 2.09 & -0.3 & 2.06 & 0.85 & 3 & 1.75 & 3.8 \\
\hline (101) $1.5 \% 5 / 25 / 2031^{*}$ & 9.96 & 12.88 & 2.89 & 5.09 & -1.28 & 1.95 & -2.95 & 3.76 \\
\hline (102) $0 \% 2 / 25 / 2019$ & 0.88 & 2.34 & 1.54 & 3.15 & 3.54 & 5.2 & 4.39 & 6.2 \\
\hline (103) $0.5 \% 5 / 25 / 2026$ & 4.22 & 5.28 & 1.52 & 2.86 & -1.96 & 4.01 & -2.99 & 4.86 \\
\hline (104) $1.25 \% 5 / 25 / 2036^{\dagger}$ & 9.06 & 14.57 & 2.11 & 8.58 & -0.71 & 3.28 & -3.09 & 3.58 \\
\hline (105) $1.75 \% 5 / 25 / 2066^{\times}$ & 19.45 & 28.35 & 9.59 & 19.24 & 8.39 & 12.86 & 5.44 & 9.23 \\
\hline (106) $0 \% 5 / 25 / 2021$ & -0.45 & 1.62 & 0.01 & 1.79 & 0.92 & 2.66 & 1.87 & 3.41 \\
\hline (107) $0.25 \% \quad 11 / 25 / 2026$ & 4.21 & 4.99 & 2.11 & 3.02 & -0.67 & 2.91 & -1.63 & 3.5 \\
\hline (108) $0 \% 5 / 25 / 2022$ & -0.95 & 1.54 & -0.74 & 1.66 & -0.95 & 2.36 & -0.56 & 2.55 \\
\hline (109) $0 \% 2 / 25 / 2020$ & 2.58 & 2.8 & 2.94 & 3.22 & 4.63 & 4.97 & 5.46 & 5.81 \\
\hline (110) $1.75 \% 6 / 25 / 2039^{\dagger}$ & 2.08 & 6.78 & -3.6 & 6.29 & -2.91 & 3.44 & -4.42 & 4.61 \\
\hline (111) $1 \% 5 / 25 / 2027$ & 5.3 & 5.45 & 3.4 & 3.55 & 0.78 & 2.1 & -0.11 & 2.14 \\
\hline (112) $2 \% 5 / 25 / 2048^{+}$ & 15.23 & 16.37 & 7.68 & 9.29 & 7.54 & 7.78 & 5.59 & 5.7 \\
\hline (113) $0 \% 3 / 25 / 2023$ & 0.29 & 1.37 & 0.62 & 1.61 & 0.3 & 1.91 & 0.81 & 2.23 \\
\hline (114) $0.75 \% 5 / 25 / 2028$ & 7.35 & 7.37 & 4.67 & 4.68 & 0.42 & 0.84 & -1.05 & 1.4 \\
\hline All yields & 2.08 & 11.96 & 1.62 & 9.85 & 2.66 & 7.86 & 2.95 & 8.01 \\
\hline Max $\log L$ & \multicolumn{2}{|c|}{$\overline{35,075.97}$} & \multicolumn{2}{|c|}{39815.22} & \multicolumn{2}{|c|}{$\overline{43,425.08}$} & \multicolumn{2}{|c|}{$44,008.49$} \\
\hline
\end{tabular}

Table 8: Summary Statistics of Fitted Errors of French Government Bond Yields cont.

The mean fitted errors and the root mean squared fitted errors (RMSE) of the yield to maturity for individual French fixed-coupon government bonds according to the independent-factors DNS model estimated on samples with varying maximum bond maturity. Asterisk * ind2̧ates fifteen-year bonds, dagger $\dagger$ indicates twenty-year bonds, plus + indicates thirty-year bonds, and cross $\times$ indicates fifty-year bonds (also highlighted in bold). All numbers are measured in basis points. 


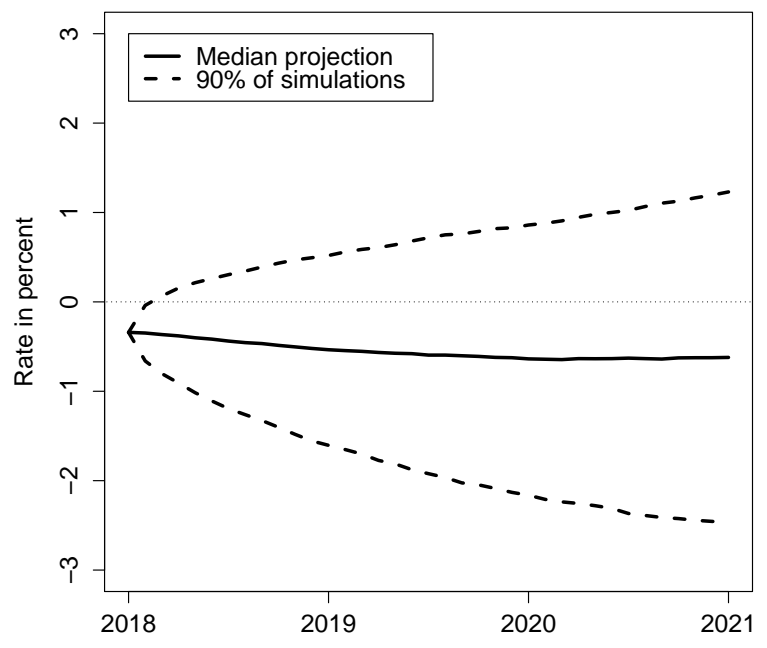

Figure 9: Short-Rate Projections from the French DNS Model

Illustration of the median and [5\%,95\%] range of the short rate from the French DNS model's simulated interest rate scenarios as of December 29, 2017.

tion the estimated parameters and factor values as of December 29, 2017. We focus on the simulated paths for the short rate, which is the model equivalent for the overnight rate targeted by the European Central Bank (ECB) and on the projected ten-year French government bond yields.

Figure 9 compares DNS forecasts of the short rate as of December 29, 2017 to provide insight into the variation in the resulting short rate projections. The figure shows the median, 5th, and 95th percentile values for the simulated short rate for a three-year forecast horizon. The median simulated short rate indicates essentially an unchanged level for the short rate over the entire forecast horizon with the short rate remaining near -0.5 percent. The upper 95th percentile indicates a rise to roughly 1 percent at three years out, while the lower 5th percentile represents further rate cuts into negative territory that pushes short rates below -2 percent by late 2020. Furthermore, the width of the range reaches almost 400 basis points by the end of 2020 .

Next, we focus on the simulated paths for the ten-year Treasury yield, which is a key benchmark for most financial institutions. Figure 10 illustrates the median and [5\%,95\%] range of the ten-year yield at each point in time from the simulated interest rate scenarios. The median simulated ten-year yield indicates a continued gradual normalization of the long- 


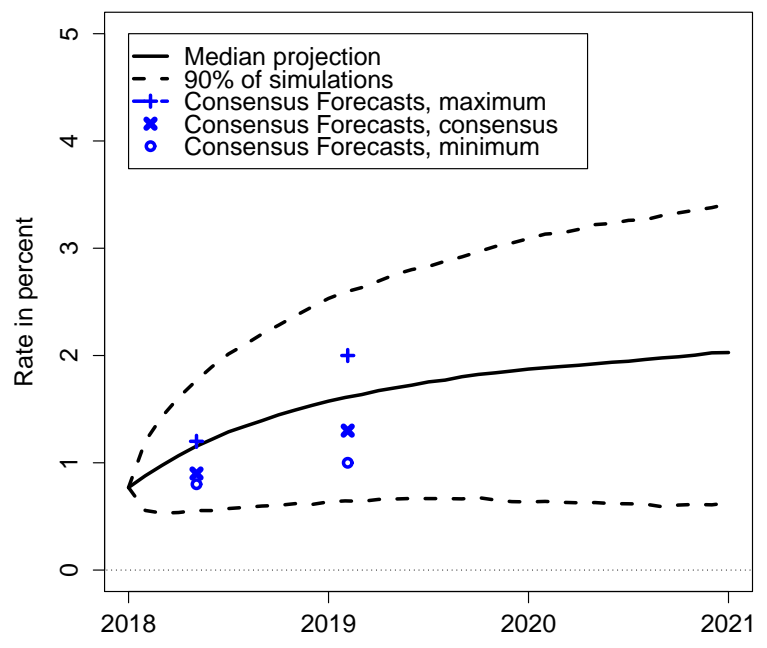

Figure 10: Ten-Year Yield Projections from the French DNS Model

Illustration of the median and [5\%,95\%] range of the ten-year yield from the French DNS model's simulated interest rate scenarios as of December 29, 2017. Also shown are the consensus forecasts as well as the maximum and minimum responses from the Consensus Forecasts survey performed on January 8, 2018.

term interest rate environment. The upper 95th percentile rises more rapidly and surpasses 3 percent at three years out, while the lower 5 th percentile represents a further decline from the already historically low levels observed at the start of the projection period.

To provide perspective for the model projections, we compare them to the Consensus Forecasts survey performed on January 8, 2018. It contains 14 responses to questions about the level of the ten-year French government bond yield at the end of April 2018 and the end of January 2019, respectively. In Figure 10, we show the consensus forecasts as well as the maximum and minimum response to those two questions. We note that the model-implied median projection is slightly more optimistic than the path of the survey's consensus forecasts. Furthermore, the $[5 \%, 95 \%]$ range of the projected ten-year yields encompasses the range of projections in the Consensus Forecasts survey. Thus, the DNS model projections suggest that the likely range of outcomes at a three-year horizon is quite wide with a width of almost 300 basis points, which is only marginally smaller than the corresponding range of projected short rates.

In Figure 11, we illustrate the entire yield curve corresponding to the three percentiles of 


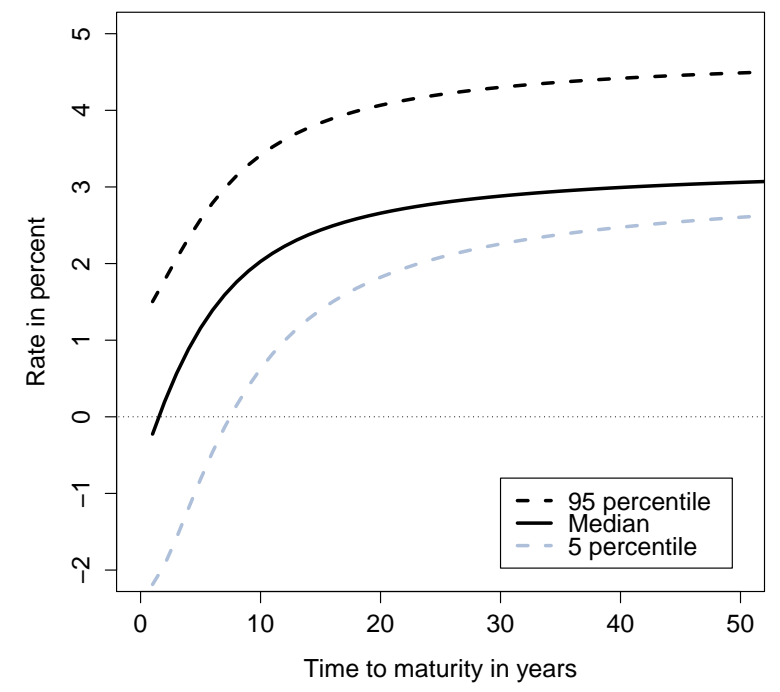

Figure 11: Simulated French Yield Curves for December 2020

the ten-year yield projections for the end of December 2020 shown in Figure 10. Note the wide variation in the shape of the yield curve that the model suggests may occur with $90 \%$ probability over the next three years. This figure underscores that the DNS model is able to generate quite a wide range of yield outcomes even at the relatively short projection horizons typically considered in regulatory stress tests of financial institutions.

\section{References}

Andersen, Torben G. and Luca Benzoni, 2010, "Do Bonds Span Volatility Risk in the U.S. Treasury Market? A Specification Test for Affine Term Structure Models," Journal of Finance, Vol. 65, No. 2, 603-653.

Cheridito, Patrick, Damir Filipović, and Robert L. Kimmel, 2007, "Market Price of Risk Specifications for Affine Models: Theory and Evidence," Journal of Financial Economics, Vol. 83, No. 1, 123-170.

Christensen, Jens H. E., Francis X. Diebold, and Glenn D. Rudebusch, 2009, “An ArbitrageFree Generalized Nelson-Siegel Term Structure Model," Econometrics Journal, Vol. 12, No. 3, C33-C64. 
Christensen, Jens H. E., Francis X. Diebold, and Glenn D. Rudebusch, 2011, "The Affine Arbitrage-Free Class of Nelson-Siegel Term Structure Models," Journal of Econometrics, Vol. 164, No. 1, 4-20.

Christensen, Jens H. E., Jose A. Lopez, and Glenn D. Rudebusch, 2014, "Can Spanned Term Structure Factors Drive Stochastic Yield Volatility?," Working Paper 2014-03, Federal Reserve Bank of San Francisco.

Dai, Qiang and Kenneth J. Singleton, 2000, "Specification Analysis of Affine Term Structure Models," Journal of Finance, Vol. 55, No. 5, 1943-1978.

Diebold, Francis X., Glenn D. Rudebusch and S. Boragan Aruoba, 2006, "The Macroeconomy and the Yield Curve: A Dynamic Latent Factor Approach," Journal of Econometrics, Vol. 131, 309-338.

Duffee, Gregory R., 2002, "Term Premia and Interest Rate Forecasts in Affine Models," Journal of Finance, Vol. 57, No. 1, 405-443.

Feldhütter, Peter, Christian Heyerdahl-Larsen, and Philipp Illeditsch, 2016, "Risk Premia and Volatilities in a Nonlinear Term Structure Model," forthcoming Review of Finance.

Harvey, A.C., 1989, Forecasting, structural time series models and the Kalman filter, Cambridge: Cambridge University Press.

Krippner, Leo, 2015, "A Theoretical Foundation for the Nelson and Siegel Class of Yield Curve Models," Journal of Applied Econometrics, Vol. 30, 97-118.

Nelson, Charles R. and Andrew F. Siegel, 1987, "Parsimonious Modeling of Yield Curves," Journal of Business, Vol. 60, No. 4, 473-489.

Thompson, Samuel, 2008, "Identifying Term Structure Volatility from the LIBOR-Swap Curve," Review of Financial Studies, Vol. 21, No. 2, 819-854. 